\title{
Redundancy-Aware Electromigration Checking for Mesh POWER GRIDS
}

\author{
by
}

\author{
Sandeep Chatterjee
}

A thesis submitted in conformity with the requirements for the degree of Master of Applied Sciences Graduate Department of Electrical \& Computer Engineering University of Toronto

(C) Copyright 2013 by Sandeep Chatterjee 


\author{
Abstract \\ Redundancy-Aware Electromigration Checking for Mesh Power Grids \\ Sandeep Chatterjee \\ Master of Applied Sciences \\ Graduate Department of Electrical \& Computer Engineering \\ University of Toronto \\ 2013
}

Electromigration (EM) is re-emerging as a significant problem in modern integrated circuits (IC). Especially in power grids, due to shrinking wire widths and increasing current densities, there is little or no margin left between the predicted EM stress and that allowed by the EM design rules. Statistical Electromigration Budgeting (SEB) estimates the reliability of the grid by considering it entirely as a series system. However, a power grid with its many parallel paths has much inherent redundancy. In this work, we propose a new model to estimate the MTF and reliability of the power grid under the influence of EM, which accounts for these redundancies. We refer to this as the mesh model. To implement the mesh model, we also develop a framework to estimate the change in statistics of an interconnect as its effective-EM current varies. The proposed algorithm is quite fast and has an overall observed empirical complexity of $O\left(n^{1.5-1.6}\right)$. The results indicate that the series model, which is currently used in the industry, gives a pessimistic estimate of power grid MTF and reliability by a factor of 3-4. 


\section{Acknowledgements}

First and foremost, I would like to thank Professor Farid N. Najm for his insightful recommendations, continuous guidance and inspiration, without which this work would not have been possible. He is the best supervisor one could ever hope for. I cherish the weekly meetings with him, in which we discussed and analyzed the progress made in my research. His excellent technical expertise and mentorship is something I aspire for. Thank you Professor, for giving me the opportunity to work with you; it has been an amazing experience that lead to my holistic development as a professional, a researcher and as a person.

I would also like to thank Professor Andreas Veneris, Professor Jianwen Zhu and Professor Tony Chan Carusone from the ECE department at the University of Toronto for reviewing this work and for providing me with constructive comments. I would also like to acknowledge the financial support provided by the University of Toronto and Natural Sciences and Engineering Research Council (NSERC) of Canada.

I am grateful to Mohammad B. Fawaz for his friendship and support. I thank him for all the good times we have had together, inside as well as outside the lab. The work presented in chapter 3 is a collaborative effort with him, and I enjoyed the fruitful discussions we had while working together.

Many thanks to Abhishek, who was my go-to-person when I was new to Toronto. I am thankful for his support during the first year of my degree program. He was always there to answer my questions on almost any topic related to my research and otherwise. I would also like to take this opportunity to thank my colleagues for providing a great and pleasant environment. I am especially grateful to Noha Sinno, Zahi Moudalla, Zissis Poulos, Jason Luu, Fitsum Assamnew, Braiden Brousseau and Jongsok Choi. I wish them best in their endeavors.

I am also lucky to have the support of my friends Aakash Nigam, Hari Sridhar, Anmol

Ratna Singh, Nitish Srivastava and many others in Toronto with whom I spent quality 
time outside my work. I will never forget the numerous Toronto adventures or the late night discussions on random topics I had with them. Also, many thanks to my friends in India for their support and motivation. I wish you all the best.

My biggest gratitude goes to my parents, Mr. Jitendra Kr. Chatterjee and Mrs. Soma Chatterjee for always encouraging me and wishing only the best for me. They never doubted my ability and invested in my future, at times compromising their present. Thank you both for all the support, this work is dedicated to you.

Lastly, I offer my regards to those whom I might have missed, but supported me in any respect during the completion of this work. 


\section{Contents}

1 Introduction $\quad 1$

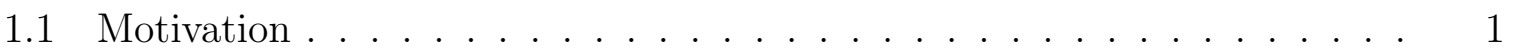

1.2 Contribution . . . . . . . . . . . . . . . . . . . 3

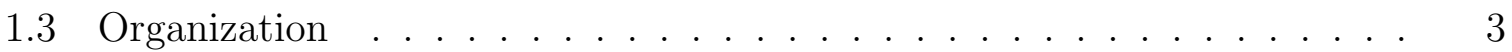

2 Background $\quad 5$

2.1 Introduction . . . . . . . . . . . . . . . . . 5

2.2 Electromigration . . . . . . . . . . . . . . . . 5

2.2.1 Electromigration Lifetime Model: Black's equation . . . . . . . 6

2.2 .2 Blech Effect . . . . . . . . . . . . . . . . . 7

2.2.3 Effective-EM Current . . . . . . . . . . . . . . . 8

2.3 EM Reliability Mathematics . . . . . . . . . . . . . . . 8

2.3.1 Basic Concepts . . . . . . . . . . . . . . . . . 9

2.3.2 EM Time-to-Failure Distributions . . . . . . . . . . . . . 10

2.3.3 Normal Distribution . . . . . . . . . . . . . . . . . . 12

2.3.4 Lognormal Distribution . . . . . . . . . . . . . . . . . . . . 14

2.3.5 TTF sample Generation . . . . . . . . . . . . . 15

2.4 Power Grid Model . . . . . . . . . . . . . . . . . . . . . 15

2.5 Monte Carlo random sampling approach . . . . . . . . . . . . . . . . 17

2.5.1 Mean Estimation ................. 18 
2.5.2 Probability Estimation . . . . . . . . . . . . . . . . 21

2.6 Electromigration checking in power grids . . . . . . . . . . . . 22

3 Mesh model for Power Grid Reliability Verification 25

3.1 Introduction . . . . . . . . . . . . . . . . . . 25

3.2 Failure model for a single interconnect . . . . . . . . . . . . . 26

3.3 New criteria for power grid failure . . . . . . . . . . . . . 26

3.4 New grid failure model: Mesh model f . . . . . . . . . . . . . . 28

3.5 Efficiently updating Voltage Drops _. . . . . . . . . . . . . . . 31

3.5 .1 Problem definition $\ldots \ldots \ldots \ldots \ldots$

3.5.2 Woodbury Formula for voltage updates . . . . . . . . . . . 32

3.5.3 Improving scalability using Banachiewicz-schur form . . . . . . 34

3.6 Experimental Results . . . . . . . . . . . . . . . . . . . . 36

3.7 A small note on the Mesh Model . . . . . . . . . . . . . . . . . . . 38

4 Estimating EM statistics for step currents 42

4.1 Introduction . . . . . . . . . . . . . . . . . . . . 42

4.2 Problem Definition . . . . . . . . . . . . . . . . . . . 42

4.3 Updating Interconnect statistics . . . . . . . . . . . . . . . . . 43

4.3.1 The Single Step Case . . . . . . . . . . . . . . . . . . 43

4.3 .2 Assumptions. . . . . . . . . . . . . . . . 45

4.3.3 Determining $F_{0}(t)$ and $\delta$ for single-step case $\ldots \ldots \ldots \ldots$

4.3.4 The Case of Multiple changes in Currents . . . . . . . . . 47

4.3.5 Incorporating the Blech Effect . . . . . . . . . . . . 50

4.3.6 Updating A TTF sample . . . . . . . . . . . . . . . . 52

4.3.7 Dropping Assumption $4.3 \ldots \ldots \ldots \ldots$

4.4 Implementation . . . . . . . . . . . . . . . . . . 55

4.4 .1 Obtaining a grid TTF sample . . . . . . . . 55 
4.4 .2 Estimating grid MTF . . . . . . . . . . . . . 55

4.5 Experimental Results . . . . . . . . . . . . . . . . . . 56

$5 \quad$ Reliability Estimation and Selective TTF updates $\quad 67$

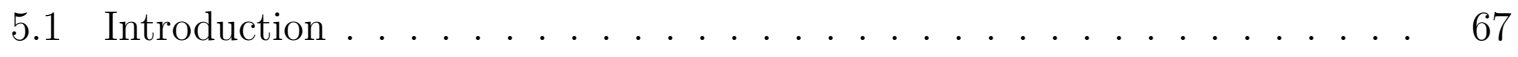

5.2 Survival Probability and Reliability Estimation _. . . . . . . . . 67

5.3 Selective TTF updates for speed-up . . . . . . . . . . . . . . . 70

5.3 .1 Motivation . . . . . . . . . . . . . . . . 70

5.3 .2 Overview of approach . . . . . . . . . . . 70

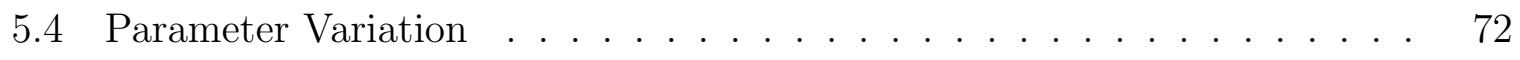

5.5 Implementation and Experimental Results . . . . . . . . . . . . 74

6 Conclusions and Future Work $\quad 82$

$\begin{array}{lr}\text { Appendices } & 84\end{array}$

$\begin{array}{lr}\text { A The Banachiewicz-schur form } & 85\end{array}$

$\begin{array}{lr}\text { Bibliography } & 86\end{array}$ 


\section{List of Tables}

3.1 Comparison between Woodbury Formula Solution Update and Sparse-LU using $U M F P A C K \ldots \ldots \ldots$. . . . . . . . . . . . . . . . . . . . . . .

4.1 Details of Power Grids used in experiments . . . . . . . . . . . . . . . 58

4.2 Comparision of Power Grid MTF $\mu_{s}$ and $\mu_{m}^{*}$ as obtained using the Series model and Mesh model for the original approach. $\mu_{s}$ and $\mu_{m}^{*}$ are estimated with $95 \%$ confidence and maximum $5 \%$ relative error. . . . . . . . . . . 60

4.3 Speed and accuracy of the secondary approach, $\mu_{m}^{\prime}$ is estimated with $95 \%$ confidence and maximum $5 \%$ relative error. . . . . . . . . . . . . 61

5.1 Survival probability Estimation of power grids for user-provided values of $y$ with $95 \%$ confidence and maximum absolute error of $\pm 0.05 . \quad$. . . . . 76

5.2 Speed and accuracy after using selective TTF update approach to estimate power grid MTF, $\overline{|\pi(\mathrm{R})|}$ is the average size of the set $\pi(\mathrm{R})$ (in terms of $\%$ of interconnects) over all failures when $\delta_{v}^{\star}=10^{-3} V$ and $\epsilon^{+}$is the percentage error in MTF estimation with respect to the original approach. . . . . . . 78 


\section{List of Figures}

1.1 Nanometer technology trends $[2] \ldots \ldots \ldots \ldots$

2.1 A typical triple point in a wire . . . . . . . . . . . 6

2.2 Typical $f(t)$ plot for the normal and the lognormal distributions . . . . 13

2.3 A typical DC model of power grid . . . . . . . . . . . . . . . . 16

2.4 Definition of $z_{\alpha / 2} \ldots \ldots \ldots \ldots \ldots$

2.5 Comparison of Statistical Bounds for 95\% and 99\% confidence, where Eq. A: $\left(z_{\alpha / 2} /(2 \mathcal{E})\right)^{2}$, Eq. B: $\left(\left(\sqrt{63}+z_{\alpha / 2}\right) /(2 \sqrt{\mathcal{E}})\right)^{2}$ and Eq C: $\left(\left(z_{\alpha / 2} \sqrt{2 \mathcal{E}+0.1}+\sqrt{(\mathcal{E}+0.1) z_{\alpha / 2}^{2}+3 \mathcal{E}}\right) /(2 \mathcal{E})\right)^{2} \ldots \ldots \ldots 22$

2.6 A typical on-die modern power grid with a mesh structure . . . . . . . 23

3.1 Resistance and void shape evolution with time $[42] \ldots \ldots \ldots$

3.2 Conductance change with EM degradation for the step-increase linear model and open-circuit model . . . . . . . . . . . . . . . . 27

3.3 Flow-chart for the mesh model . . . . . . . . . . . . . . . . . . . . . 29

3.4 Comparison of average time taken to update the voltage drops . . . . . 38

3.5 Comparison of cumulative time taken to update the voltage drop after $p^{\text {th }}$ interconnect fails for grid IBMPG4 . . . . . . . . . . . . . 39

3.6 Empirically determined complexity of (a) Sparse-LU (UMFPACK) (b) Woodbury and Woodbury-Banachiewicz-Schur form . . . . . . 39 
3.7 Voltage-drop maps of IBMPG5 grid (a) at $t=0$, when it is fresh and (b) after the first interconnect failure. As per series model, (b) has failed, but clearly, the voltage drops at all nodes are below $v_{t h}=0.18 \mathrm{~V} \ldots$. . . .

3.8 Voltage-drop maps of IBMPG6 grid (a) at $t=0$, when it is fresh and (b) after the first interconnect failure. As per series model, (b) has failed, but clearly, the voltage drops at all nodes are below $v_{t h}=0.18 \mathrm{~V}$. . . . . .

4.1 Proposed approach for single-step case, (a) Current density profile of $S_{0}$, (b) Current density profile of $S_{1}$, (c) Known CDF $F_{1}(t), \mathrm{CDF} F_{0}(t)$ unknown for $t>t_{1},(\mathrm{~d})$ The proposed solution . . . . . . . . . . . 44

4.2 Proposed approach for multi-step case, (a) CDF plot of $S_{0}$ as per the proposed approach, the square markers locate the time of current change when a new shifted lognormal is used to describe the statistics, (b) Current density profile . . . . . . . . . . . . . . . . . .

4.3 Proposed approach for multi-step case, with EM-immune time-spans in between (a) CDF plot of $S_{0}$ as per the proposed approach (b) Current density profile . . . . . . . . . . . . . . . .

4.4 Updating a TTF sample (a) Obtaining a TTF sample at $t=0$ (b) Updating TTF sample when current density changes, the square marker locates the time of current-density change . . . . . . . . . . . . . .

4.5 Comparison of MTF as obtained using the series model and mesh model for the original approach . . . . . . . . . . . . . . .

4.6 Dependence of relative error between $\mu_{m}^{\prime}$ and $\mu_{m}^{*}$ on $\left(v_{t h}-\max \left(v_{0}\right)\right)$ and $\sigma_{\ln }$ for IBMPG2 grid . . . . . . . . . . . . . . . .

4.7 Average time-breakup between node voltage updates and TTF updates of surviving interconnects after an interconnect failure . . . . . . . . . .

4.8 Scalability of the original approach . . . . . . . . . . . . . . 
4.9 Voltage drop maps of IBMPGNEW2 (a) at $t=0$ and (b) at the time of failure grid failure. For this particular realization, 68 interconnect failures were required to exceed threshold voltage $v_{t h}=0.18 \mathrm{~V}$. . . . . . . .

4.10 Voltage drop maps of IBMPG5 (a) at $t=0$ and (b) at the time of failure grid failure. For this particular realization, 20 interconnect failures were required to exceed threshold voltage $v_{t h}=0.18 \mathrm{~V} \ldots \ldots$. . . . . .

4.11 Voltage drop maps of IBMPG6 (a) at $t=0$ and (b) at the time of failure grid failure. For this particular realization, 36 interconnect failures were required to exceed threshold voltage $v_{t h}=0.18 \mathrm{~V} \ldots \ldots$. . . . . .

5.1 Change in node voltage drops for a randomly chosen single interconnect failure in power grids IBMPG5, IBMPG6, IBMPGNEW2 and G4. Nodes where change in voltage drop is less than $10^{-3}$ are omitted in the figure. . 71

5.2 Dependence of boundary of $\pi(\mathrm{R})$ on $\delta_{v}^{\star}, s=\partial v_{k} / \partial \mathrm{R} \ldots \ldots . \ldots 72$

5.3 Change in gain ratio with current exponent $(n)$ and standard deviation of lognormal $\left(\sigma_{\ln }\right)$ for power grid IBMPG2 . . . . . . . . . . . . 74

5.4 Variation of gain ratio with property of metal used for power grid IBMPG2 75

5.5 Variation of gain ratio with power grid configuration for power grid IBMPG2 75

5.6 Estimated statistics of RVs $\mathbf{T}_{s}$ and $\mathbf{T}_{m}$ for IBMPG5, they are found to have a normal distribution . . . . . . . . . . . . . . . . . . 77

5.7 Scalability of the selective TTF update approach. . . . . . . . . . . . . . 80

5.8 Runtime $\left(t_{c p u}\right)$ and error $\left(\left|\varepsilon^{+}\right|\right)$vs. $\delta_{v}^{\star}$; effect of varying $\delta_{v}^{\star}$ on $\overline{t_{f}}$ (average time/failure) and $\overline{|\pi(\mathrm{R})|}$ (the average cardinality of the set $\pi(\mathrm{R}))$. All the results are for IBMPG6. . . . . . . . . . . . . . . . . . 


\section{Chapter 1}

\section{Introduction}

\subsection{Motivation}

Verifying the power grid is a crucial step in VLSI design, as the reliability of the underlying logic heavily depends on its power grid. Not only must an IC perform as desired, it must also survive and function as intended for several years before failing. This concern is addressed by a variety of design measures, for example the choice of materials, the length and width of interconnects and so on. The density of on-chip interconnects has increased from generation to generation of modern integrated circuits. This requires a decrease in both interconnect width and thickness and, consequently, the operating current densities increase. As such, new factors come to bear, which reduce reliability and which previously were negligible. Specifically, electromigration (EM) has re-emerged as a significant problem in modern chip design and there are three problems that demand attention: 1) existing EM checking techniques for the power grid are overly pessimistic (because of an underlying series system assumption, as will be explained later), leading to loss of safety margins and multiple design iterations, 2) increased current density in grid metal lines has led to a significant loss of margins between the predicted EM stress and the allowed thresholds, and 3) checking modern, large power grids for EM has become very expensive. To make things worse, it is forecast [1] [2] that metal line current density and reliability due to EM will get dramatically worse with continued technology scaling. As a result, EM signoff has become increasingly difficult and designers are forced to reconsider traditional approaches, and to look with suspicion at the large safety margins and pessimism built into traditional EM checking methods.

Historically, 'worst case' current density limits for individual lines were used to arrive at reliable designs for ICs. However, since it is never the case that all the lines in power grids will carry the worst case currents at the same time, the 'worst case' current density 

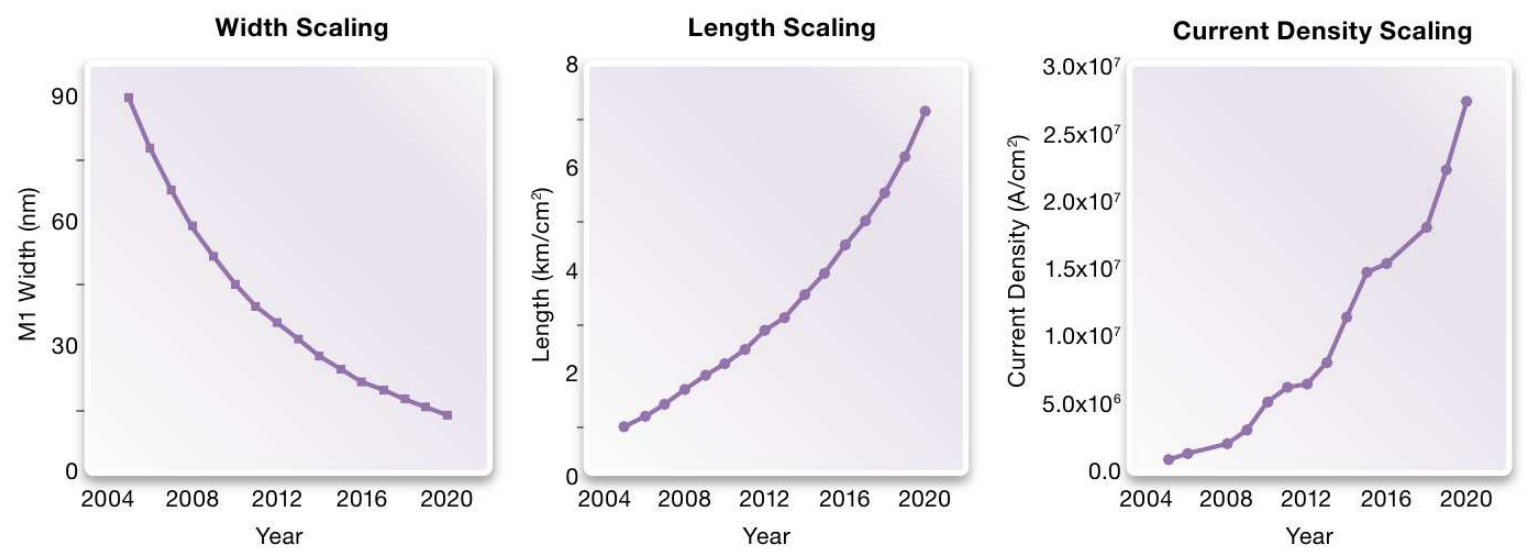

Figure 1.1: Nanometer technology trends [2]

approach severely restricted the design process. This motivated the need for a model to relate the reliability of individual components to the reliability of the entire system. In an early contribution [3] a series model was proposed to determine the reliability of an IC, under which a system is deemed to have failed as soon as any of its components fails. Under the series model, and with some simplifying assumptions, the failure rate of the system is the sum of failure rates of individual components. The series model was applied to the Alpha 21164 microprocessor, under the name Statistical Electromigration Budgeting (SEB) [4] and became a standard technique in many industrial CAD tools.

However, modern power grids use a mesh structure. As such, there are many paths for the current to flow from the C4 bumps to underlying logic, a characteristic we refer to as redundancy. Mesh power grids have much redundancy and are in fact closer to (but not quite) a parallel system, rather than a series system; and so have a longer lifetime than a series system. This issue has largely been ignored in EM checking tools, both in academia and industry; no industrial tool has this feature today. While SEB accounts for the fact that EM failures are statistical it does not, however, recognize the benefits of redundancy and it treats the overall metal structure as a series structure. A power grid is not necessarily failed if one of its metal line fails. Instead, in our approach, we deem a power grid to have failed only when enough lines have failed that the voltage on the grid becomes unacceptable. Our data shows that, in many cases, a grid can tolerate up to 70 or more line failures before it truly fails! The results obtained using publicly available grids from IBM, with up to $700 \mathrm{k}$ nodes, and internally generated grids, with up to 1 million nodes, show an increase in predicted lifetime of 3-4X compared to the existing series-system based approach. A lot of margin is therefore "left on the table" and there is room for high-impact improvements in EM verification. 


\subsection{Contribution}

The goal of this research is to develop a more realistic power grid EM checking and budgeting method that takes the redundancy of the power grid structure into account. In this work, we make an attempt to move away from the overly pessimistic series model to a new model for estimating the mean time-to-failure of the power grid. We also connect the IR-drop with EM degradation to determine when a power grid is presumed to have failed. Specifically,

1. We develop a new model, referred to as the mesh model, that factors in the redundancy of the power grid while estimating its MTF and reliability. In implementing the mesh model, we also develop an efficient exact method to update the node voltage drops as the structure of the grid changes due to failure of interconnects.

2. We propose a novel framework to estimate the change in statistics of an interconnect as its effective-EM current density varies in steps on a time-scale comparable to the failure times of interconnects, as is the case in mesh model.

\subsection{Organization}

The thesis is organized as follows: Chapter 2 covers the necessary background material on electromigration and the associated reliability mathematics. It then familiarizes the reader with the power grid model used and describes the Monte-carlo random sampling method used for mean and probability estimation. Relevant prior work in electromigration checking is also reviewed in this chapter. Chapter 3 presents the mesh model in detail. Among other things, an implementation of the mesh model needs to repeatedly a) solve a large linear system to find the new node voltage drops and b) update the failure statistics of the surviving interconnects, as lines fail due to electromigration. The second part of chapter 3 tackles the issue of finding new voltage drops: it presents a highly efficient exact method based on the Woodbury formula and the BanachiewiczSchur form to update the node voltage drops as the conductance matrix $G$ undergoes low rank updates. The second issue is dealt in chapter 4 , which proposes a novel framework to update the failure statistics of the surviving interconnects. In Chapter 5, we first extend the framework developed so far to estimate the probability of survival of a power grid for a given period of time based on Monte-carlo random sampling approach. Next, we describe a heuristic approach to speed-up the MTF estimation engine by performing selective TTF updates. Finally, it is shown that the reduction in pessimism as predicted 
by the mesh model is stable under parameter variation. We conclude and give future research directions in Chapter 6. 


\section{Chapter 2}

\section{Background}

\section{$2.1 \quad$ Introduction}

In this chapter, we will review the basic background material. Section 2.2 reviews the key literature on electromigration relevant to this work. In the next section, basic probability terms essential for understanding the stochastic reliability analysis are defined. In section 2.4, we focus on the power grid model to be used. In section 2.5, we turn our focus to the Monte Carlo random sampling approach, where we derive the stopping criteria (in terms of number of samples to be obtained) for estimating the mean of a distribution or the probability of occurrence of an event. Finally, in section 2.6, we briefly review the previous electromigration checking techniques used for power grids.

\section{$2.2 \quad$ Electromigration}

Electromigration is the mass transport of metal due to momentum transfer between electrons (driven by an electric field) and diffusing metal atoms. Failure occurs in metal lines only when there is a flux divergence with regard to the movement of metal atoms. It commonly occurs at distortions in the lattice, in the form of vacancies and/or grain boundaries which can be schematically represented by triple points (see Figure 2.1). These allow for diffusion and/or migration of metal atoms that lead to flux divergence. Flux divergence arises when the flow of metal atoms into the region is not equal to the outflow of atoms from the region. A void is created due to depletion (when outflow from a region is greater than the flow into it) and a hillock is created due to accumulation (when the flow into a region is greater than the outflow). 


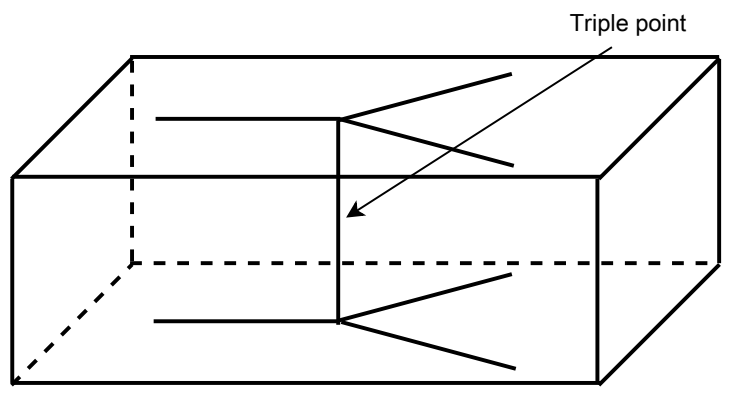

Figure 2.1: A typical triple point in a wire

\subsubsection{Electromigration Lifetime Model: Black's equation}

Under the influence of Electromigration, metal line resistance increases as a line approaches failure and starts to deform due to void creation or hillock formation. Empirically, Black's equation has been used to determine the Mean Time to Failure or MTF of a metal line under the influence of electromigration [5]:

$$
M T F=\frac{w_{d} t}{A} J^{-n} \exp \left(\frac{E_{a}}{k T_{m}}\right)
$$

where $A$ is an empirical constant that depends on a host of physical properties such as volume resistivity of the metal and effective ionic scattering cross section for electrons, $w_{d}$ and $t$ are the width and thickness of the line respectively, $J$ is the effective-EM current density, $n$ is a current exponent, $k$ is the Boltzmann's constant, $T_{m}$ is the temperature in Kelvin and $E_{a}$ is the activation energy for electromigration. Since the power grid is composed of layers made up of the same metal, the physical properties used to arrive at $A$ remain constant throughout the grid, implying that $A$ is simply a scaling factor in (2.1). The activation energy $E_{a}$ and current exponent $n$ are empirically determined. A current density exponent $n=1$ is consistent with void growth limited failure since the rate of unconstrained void growth is proportional to the current density [6][7]. An exponent $n=2$ indicates void nucleation limited failure in the model based on the Korhonen analysis [8] and other theoretical works [9][10]. If $1<n<2$, then failure is due to a combination of nucleation and growth [11] [12], but if $n>2$, the failure is due to Joule heating induced temperature gradients or due to short length effects [12].

Voids may lead to open-circuits or unacceptable resistance increase in a line, whereas protrusion of hillocks usually cause short-circuits between adjacent lines and inter-level conductors at positions where conductors cross over each other or in devices where there are two spaced layers, such as a capacitor [13]. Hillocks due to EM can also result in thin dielectrics, which are further susceptible to dielectric breakdown [14]. In this work, we 
assume for simplicity that all interconnect failures due to EM are caused by nucleation and/or growth of voids.

\subsubsection{Blech Effect}

Electromigration is mainly caused due to divergence of atomic flux induced by the electron wind. This atomic flux is opposed by the back-stress developed due to accumulation of atoms at the anode end. If the length of line is short enough, then this back stress could overcome the critical stress required for nucleation of a void in a line (or even stop the growth of void in a line), and thus the line is no longer susceptible to EM failure. Blech was the first to observe this phenomenon [15]. He designed an experiment where conductor islands were deposited onto a titanium nitride (TiN) film and stressed at a high current density. He noted that when the stripe was reduced to a certain length, the metal transport stopped. This observation was explained by considering the driving force of atomic flux as a combination of two forces: the electron wind force and the mechanical back-stress force. The atomic flux can then be expressed as [15] [16] [17] [18]:

$$
J_{a}=\frac{D_{a} C_{a}}{k T}\left(F_{\text {elec }}+F_{\text {mech }}\right)=\frac{D_{a} C_{a}}{k T}\left(\left|Z^{*}\right| e \rho J-\Omega \frac{\partial \Lambda}{\partial x}\right)
$$

where $D_{a}$ is atomic diffusivity, $C_{a}$ is atomic concentration, $\Lambda$ is the hydrostatic stress, $\Omega$ is the atomic volume, $\rho$ is the resistivity of the material, $Z^{*}$ is the effective atomic charge and $e$ is the fundamental atomic charge. In steady state, the electron wind force balances the mechanical back-stress force, so that $J_{a}=0$, and the stress gradient is given as:

$$
\frac{\partial \Lambda}{\partial x}=\frac{\left|Z^{*}\right| e \rho J}{\Omega}
$$

Integrating (2.2) over the length of line yields:

$$
\Lambda(x)=\Lambda_{0}+\frac{\left|Z^{*}\right| e \rho J}{\Omega} x
$$

where $\Lambda_{0}$ is the stress at $x=0$. Equation (2.4) implies that the maximum stress-gradient developed in an interconnect is determined by its current density. Hence, if the current density changes, the stress-gradient changes.

Given that $\Lambda_{n u c l}$ is the hydrostatic stress required for void nucleation, a critical product for electromigration failure can be stated as:

$$
\beta_{c}=(J L)_{c}=\frac{\Omega\left(\Lambda_{\text {nucl }}-\Lambda_{0}\right)}{Z^{*} e \rho}
$$


In other words, for a given interconnect that has not already failed, if the product of its current density $(J)$ and length $(L)$ is less than than $\beta_{c}$, the maximum stress developed due to the present current density is not enough for a void to nucleate, which means that the interconnect is immune to electromigration failure. This effect is referred as the Blech effect and $\beta_{c}$ is the critical Blech product. For a given current density $J$, a line is said to be EM-immune if $J L \leq \beta_{c}$ and EM-susceptible if $J L>\beta_{c}$.

\subsubsection{Effective-EM Current}

Because EM is a long-term cumulative failure mechanism, the changes in line current on very short time-scales (such as the normal operation of a digital chip), are not terribly significant. Instead, the standard approach is to compute an effective-EM current, which is a constant current value, derived from the line current waveform that gives the same lifetime for that line under the influence of electromigration. In traditional EM work, this effective current is computed based on some assumed periodic current waveform. If the waveform is uni-directional, then Direct Current EM analysis is used, based on time-averaged current density $\left(J_{\text {avg }}\right)[19]$.

$$
J_{\mathrm{DC}, \mathrm{eff}}=J_{\text {avg }}=\frac{1}{T} \int_{0}^{T} J(t) d t
$$

For more general cases of bi-polar currents, transient current EM analysis uses an effective-EM current density of [20]:

$$
J_{\text {tran,eff }}=\frac{1}{T}\left(\int_{0}^{T} J^{+}(t) d t-\varphi \int_{0}^{T}\left|J^{-}(t)\right| d t\right)
$$

where $\int J^{+}(t) d t$ is the integral of one side of the current waveform and is larger than the opposite side (i.e. $\int J^{-}(t) d t$ ) and $\varphi$ is the EM recovery factor, that is determined experimentally.

\subsection{EM Reliability Mathematics}

The Time-to-Failure (TTF) of a line under the influence electromigration is subject to manufacturing variations. To elaborate, consider an experiment E1 with a large population set $S$, of $N$ conductors (lines), made of the same material, using the same manufacturing process and with identical dimensions. All the conductors are stressed with the same current density $J$ at a temperature $T_{m}$ for time $t \geq 0$. Then, it is found that the 
TTF of all the conductors are not exactly the same. Instead, it is observed that the TTF of the conductors in $S$ are distributed over some time-range. In other words, if we pick any single conductor from the set $S$, the only thing we know is the frequency distribution of the TTFs obtained from the experiment E1. The notion of random variable is used to (rigorously) deal with such scenarios: one can view the different TTFs obtained from the population as different realizations of a single conductor, whose TTF is modeled as a random variable. In this section, basic probability terms and concepts essential for understanding the stochastic reliability analysis of EM are presented.

\subsubsection{Basic Concepts}

The concept of a random variable, combined with the four basic probability functions Cumulative Distribution Function (CDF), Probability Distribution Function (PDF), Reliability Function and Failure/Hazard rate Function are the fundamental building blocks of reliability mathematics used to describe a non-deterministic (random) process, such as degradation due to EM.

Random Variable : A Random Variable or RV is a function that maps the outcome of a random process to a real number. In this document, they are represented by bold face capital letters. RVs can be classified as either continuous or discrete. Because the TTF of a single line can assume any value in a given time-range, it is described by a continuous RV, henceforth denoted by $\mathbf{T}$. In formal terms, $\mathbf{T}$ stands for the TTF of a single randomly chosen line such that the sets $\{\mathbf{T} \leq t\}$ represent the events that the line has failed before time $t$, and have assigned probability values. An RV can be completely characterized by its PDF or CDF, so that there is no further need to refer to the underlying probability space [21].

Cumulative Distribution Function : A Cumulative Distribution Function or CDF of a RV $\mathbf{T}$, denoted by $F(t)$ is defined as:

$$
F(t) \triangleq \mathcal{P}\{\mathbf{T} \leq t\}
$$

where the right hand side represents the probability that the RV $\mathbf{T}$ takes a value less than or equal to $t$. In other words, the probability that a single randomly chosen conductor will fail by time $t$ is given by $F(t) . F(t)$ is a monotonically increasing function in $t$, with $F(-\infty)=0$ and $F(\infty)=1$. The RHS of (2.8) can be evaluated if the type of distribution, the mean and the standard deviation of $\mathbf{T}$ are known beforehand, or if we have a sufficient number of TTF samples. 
Probability Distribution Function : A Probability Distribution Function or PDF of a RV $\mathbf{T}$ is a function, denoted by $f(t)$, defined as:

$$
f(t) \triangleq \frac{d F(t)}{d t}
$$

The probability for $\mathbf{T}$ to fall within a particular interval $\left[t_{1}, t_{2}\right]$ is given by the integral of the PDF over that region:

$$
\mathcal{P}\left\{t_{1}<\mathbf{T} \leq t_{2}\right\}=F\left(t_{2}\right)-F\left(t_{1}\right)=\int_{t_{1}}^{t_{2}} f(t) d t
$$

Reliability function : A Reliability Function, denoted by $R(t)$, of a RV $\mathbf{T}$ is defined as:

$$
R(t) \triangleq 1-F(t)=\mathcal{P}\{\mathbf{T}>t\}
$$

where the right hand side represents the probability that the RV $\mathbf{T}$ takes a value greater than $t$. In other words, $R(t)$ is the probability that the single randomly chosen conductor will survive beyond time $t . R(t)$ is a monotonically decreasing function in $t$, with $R(-\infty)=1$ and $R(\infty)=0$.

Failure rate : The Failure rate, $\lambda(t)$ is defined as the (instantaneous) rate of failure for the survivors up to time $t$ during the next instant of time [22]. Mathematically:

$$
\begin{aligned}
\lambda(t) & \triangleq \lim _{\Delta t \rightarrow 0} \frac{\text { Probability of failure in }[t, t+\Delta t], \text { given still operational at } t}{\Delta t} \\
& =\lim _{\Delta t \rightarrow 0} \frac{\mathcal{P}\{t<\mathbf{T} \leq t+\Delta t\}}{\Delta t \cdot \mathcal{P}\{\mathbf{T}>t\}} \\
& =\frac{1}{R(t)} \lim _{\Delta t \rightarrow 0} \frac{F(t+\Delta t)-F(t)}{\Delta t}=\frac{f(t)}{R(t)}
\end{aligned}
$$

Thus, the failure rate, or the hazard rate, describes the conditional probability of failure around a time point $t . \lambda(t)$ is instantaneous, it may change the next instant, and the units that have already failed play no further role since only the survivors count.

\subsubsection{EM Time-to-Failure Distributions}

The type of distribution function used to characterize the lifetimes arising from a wide range of failure mechanisms (including electromigration), can be established in two ways:

1. Theoretical approach: In this approach, the lifetime distribution model is chosen 
based on the physics of failure mechanism. This is the ideal approach, but in general, it is very hard to derive the distribution from the underlying interactions. Hence, the second approach is preferred.

2. Empirical approach: The frequency distribution obtained from any experiment (such as E1 on page 8), when normalized with respect to the total number of conductors $N$, gives us a (coarse) staircase approximation of the PDF. Equation (2.10) can then be used to find the CDF $F(t)$ by setting $t_{1}=0$ and $t_{2}=t$. There is another easier way to find the CDF $F(t)$ : define $N_{\text {failed }}(t)$ to be the number of conductors that have failed in the time-period $[0, t]$. Then, from definition, the $\mathrm{CDF}$ is given by:

$$
F(t) \approx \frac{N_{\text {failed }}(t)}{N}
$$

As $N$ is increased, the normalized frequency distribution of TTF samples approaches the actual PDF and RHS of (2.13) approaches the actual CDF. However, since $N$ cannot be increased beyond a limit, the actual practice in industry is to use goodness-of-fit methods on the experimental data to choose one of known distribution models as the lifetime distribution model for a given failure mechanism.

For electromigration, there have been many (theoretical and experimental) investigations regarding which distribution model to follow. Venables et. al. [23] present a model that relates the time to failure due to EM to the density of initial voids in the metal stripe. Using an experimentally observed distribution of this density they derive the corresponding distribution of time to failure which turned out to be very close to the lognormal distribution. Gall et. al. [24] studied the early failures due to EM on a total of 20, 000 interconnects, and showed that lognormal is valid. Lloyd et. al. [25] explain the lognormal observed behavior of electromigration as the result of a normal distribution of activation energies $\left(E_{a}\right)$ and a lognormal distribution of grain sizes. However, it is pointed out that the lognormal is not extendable (failure time of a series system of lognormal life elements is not lognormal), and the multi-lognormal (an extreme value lognormal) is more appropriate. Yoh et. al. [26] propose a shifted-lognormal (SLN) as the lifetime distribution model of individual long wires, and showed that shifted lognormal and truncated multi-lognormal are very similar. However, the lognormal still remains a practical and simple model for the Time-to-failure of long metal lines, and is used throughout this work.

Another justification for the use of lognormal as time to failure distribution model of long metal lines comes from the fact that degradation due to EM is mainly due to diffusion or migration of atoms, which is expected to follow multiplicative degradation 
property. If at any instant in time a degradation process undergoes a small increase in the total amount of degradation that is proportional to the current total amount of degradation, then it is reasonable to expect the time to failure (i.e. reaching a critical amount of degradation) to follow a lognormal distribution [22]. This can be formally explained as follows:

Let $y_{1}, y_{2}, \ldots y_{n}$ be the amount of degradation for a particular failure process taken at successive discrete instants of time as the process moves towards failure. Assume that the following relationship exists between the measured quantities:

$$
\begin{aligned}
y_{i}-y_{i-1} & =\eta_{i} y_{i-1} \\
y_{i} & =\left(1+\eta_{i}\right) y_{i-1}
\end{aligned}
$$

where $\eta_{i}$ are random independent shocks to the system that move the degradation process forward; then the degradation at the $n^{\text {th }}$ instant is given by:

$$
y_{n}=\left[\prod_{i=1}^{n}\left(1+\eta_{i}\right)\right] y_{0}
$$

where $y_{0}$ is a constant. Taking natural logarithms on both sides of (2.15):

$$
\ln y_{n}=\sum_{i=1}^{n} \ln \left(1+\eta_{i}\right)+\ln y_{0} \approx \sum_{i=1}^{n} \eta_{i}+\ln y_{0}
$$

From the Central Limit Theorem, it can be asserted that $\sum_{i=1}^{n} \eta_{i}$ (and $\ln y_{n}$ ) has approximately a normal distribution [27], and thus by the properties of lognormal distribution (as presented in section 2.3.4), $y_{n}$ has a lognormal distribution for any $n$ (or at any continuous time $t$ ). Typically, failure happens when degradation reaches a critical predefined point, and and thus the time of failure caused by a multiplicative degradation process can be modeled by a lognormal distribution.

\subsubsection{Normal Distribution}

The normal distribution, also called the Gaussian distribution, is one of the best known and most widely used distributions. If a RV $\mathbf{T}$ has a normal distribution, then its PDF is bell shaped, and is given by:

$$
f(t)=\frac{1}{\sigma \sqrt{2 \pi}} e^{-\frac{1}{2}\left(\frac{t-\mu}{\sigma}\right)^{2}}, \quad-\infty<t<+\infty
$$



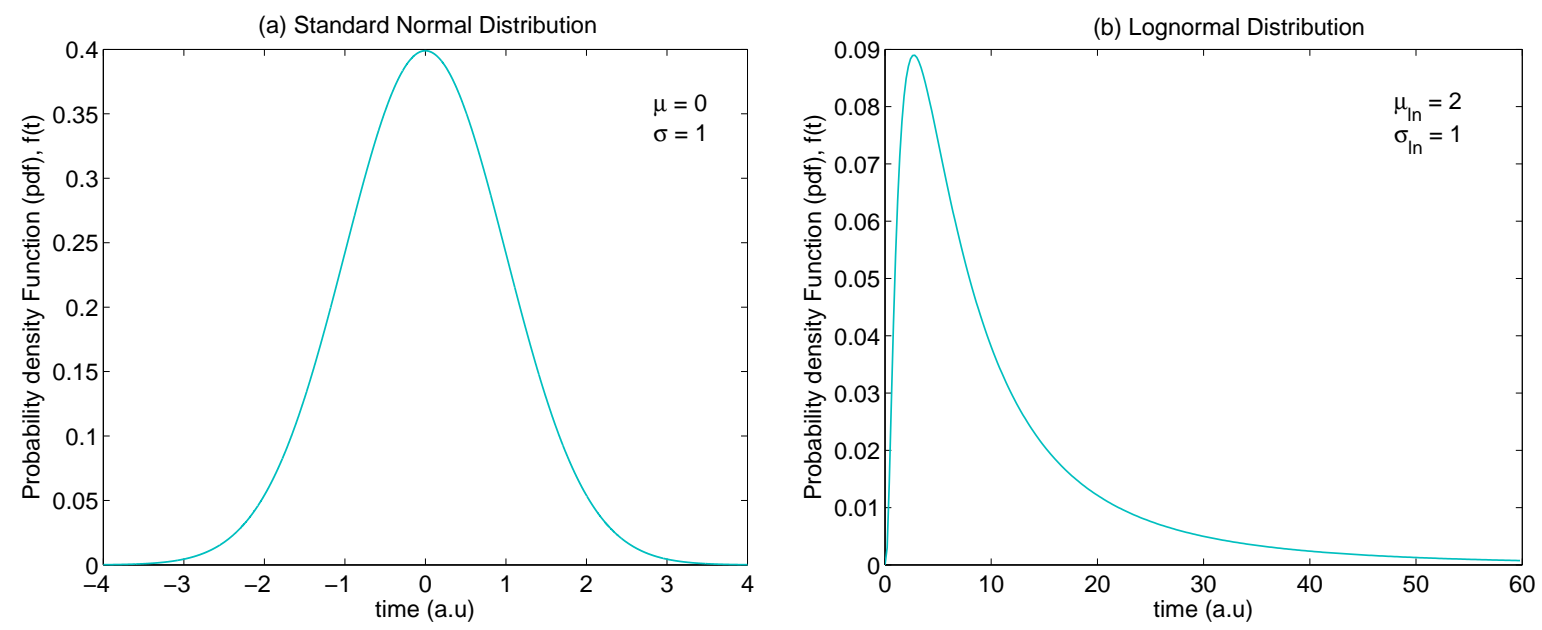

Figure 2.2: Typical $f(t)$ plot for the normal and the lognormal distributions

where $\mu \triangleq E[\mathbf{T}]$ and $\sigma^{2} \triangleq \operatorname{Var}(\mathbf{T})$ (Var denotes variance). The PDF is symmetrical around $\mu$ and attains its maximum value of $\frac{1}{\sigma \sqrt{2 \pi}} \approx \frac{0.4}{\sigma}$ at $t=\mu$. When $\mu=0$ and $\sigma=1$, the resulting normal distribution is called standard normal distribution, and its $\mathrm{PDF}$ and $\mathrm{CDF}$ are given by:

$$
\phi(t)=\frac{1}{2 \pi} e^{-\frac{1}{2} t^{2}}, \quad \Phi(t)=\int_{-\infty}^{t} \frac{1}{2 \pi} e^{-\frac{1}{2} t^{2}} d t \quad-\infty<t<+\infty
$$

If $\mathbf{T}$ is normal with mean $\mu$ and variance $\sigma$, then the RV $\mathbf{Z}=\frac{\mathbf{T}-\mu}{\sigma}$ is a $\mathrm{RV}$ with standard normal distribution. Thus, CDF of $\mathbf{T}$ can be denoted as:

$$
F(t)=\Phi\left(\frac{t-\mu}{\sigma}\right)
$$

There is no closed form expression for $\Phi$. However, on computers, it can be evaluated with the aid of the erf and erfc functions. The CDF and failure rate of the normal distribution can thus be written as:

$$
\begin{aligned}
& F(t)=\frac{1}{2}\left[1+\operatorname{erf}\left\{\frac{t-\mu}{\sigma \sqrt{2}}\right\}\right] \\
& \lambda(t)=\sqrt{\frac{2}{\pi \sigma^{2}}} \frac{e^{-\frac{1}{2}\left(\frac{t-\mu}{\sigma}\right)^{2}}}{\operatorname{erfc}\left\{\frac{t-\mu}{\sigma \sqrt{2}}\right\}}
\end{aligned}
$$




\subsubsection{Lognormal Distribution}

A random variable $\mathbf{T}$ is said to have a lognormal distribution when $\ln \mathbf{T}$ has a normal distribution. The Probability Distribution Function of a lognormal distribution is given by:

$$
f(t)=\frac{1}{t \sigma_{\ln } \sqrt{2 \pi}} e^{-\frac{1}{2}\left(\frac{\ln t-\mu_{\ln }}{\sigma_{\ln }}\right)^{2}}, \quad 0<t<+\infty
$$

where $\mu_{\ln } \triangleq E[\ln \mathbf{T}]$ and $\sigma_{\ln }^{2} \triangleq \operatorname{Var}(\ln \mathbf{T})$. A lognormal RV $\mathbf{T}$ can be transformed to a standard normal variable $\mathbf{Z}$ where $\mathbf{Z}=\left(\ln \mathbf{T}-\mu_{\ln }\right) / \sigma_{\ln }$. Hence, the Cumulative Distribution Function can be written as:

$$
\begin{array}{rlr}
F(t) & =\int_{-\infty}^{t} \frac{1}{x \sigma_{\ln } \sqrt{2 \pi}} e^{-\frac{1}{2}\left(\frac{\ln x-\mu_{\ln }}{\sigma_{\ln }}\right)^{2}} d x \\
& =\Phi\left(\frac{\ln t-\mu_{\ln }}{\sigma_{\ln }}\right) \quad 0<t<+\infty
\end{array}
$$

where $\Phi$ is the CDF of Standard Normal Distribution. If the distribution originates at some time $t=\Delta \neq 0$, then the modified PDF and CDF functions are written as:

$$
\begin{array}{ll}
f(t-\Delta)=\frac{1}{(t-\Delta) \sigma_{\ln } \sqrt{2 \pi}} e^{-\frac{1}{2}\left(\frac{\ln (t-\Delta)-\mu_{\ln }}{\sigma_{\ln }}\right)^{2}} & \Delta<t<+\infty \\
F(t-\Delta)=\Phi\left(\frac{\ln (t-\Delta)-\mu_{\ln }}{\sigma_{\ln }}\right) & \Delta<t<+\infty
\end{array}
$$

Note that $\Delta>0$ implies that a distribution originated after $t=0$, while $\Delta<0$ means that the distribution originated before $t=0$.

Define $\mu_{\mathrm{T}} \triangleq E[\mathbf{T}]$ and $\sigma_{\mathrm{T}}^{2} \triangleq \operatorname{Var}[\mathbf{T}]$. The relations between $\mu_{\ln }, \sigma_{\ln }, \mu_{\mathrm{T}}$ and $\sigma_{\mathrm{T}}$ are as follows:

$$
\begin{aligned}
\sigma_{\ln }^{2} & =\ln \left(1+\left(\frac{\sigma_{\mathrm{T}}}{\mu_{\mathrm{T}}}\right)^{2}\right) \\
\mu_{\ln } & =\ln \left(\mu_{\mathrm{T}}\right)-\frac{1}{2} \ln \left(1+\left(\frac{\sigma_{\mathrm{T}}}{\mu_{\mathrm{T}}}\right)^{2}\right)=\ln \left(\mu_{\mathrm{T}}\right)-\frac{1}{2} \sigma_{\ln }^{2} \\
\mu_{\mathrm{T}} & =\exp \left(\mu_{\ln }+0.5 \sigma_{\ln }^{2}\right) \\
\sigma_{\mathrm{T}}^{2} & =\mu_{\mathrm{T}}^{2}\left(\exp \left(\sigma_{\ln }^{2}\right)-1\right)
\end{aligned}
$$

In the case of electromigration, the MTF or $\mu_{\mathrm{T}}$ of the lognormal distribution is given by Black's equation (2.1). The deviation of $\ln \mathbf{T}$, denoted by $\sigma_{l n}$, is dependent on the ratio of width to median grain size of the metal line for aluminum [28] and on the product 
$J L^{2}$ for copper [29], and this dependence is determined experimentally. However, the deviation in both cases eventually levels off to a constant value. Hence, in this work, it is assumed that $\sigma_{l n}$ is constant for a given material, as is typical in the literature [30] [31].

\subsubsection{TTF sample Generation}

In order to generate samples for a RV $\mathbf{T}$ that has a lognormal distribution, we note that $\ln \mathbf{T}$ has a normal distribution, so that $\mathbf{Z}=\frac{\ln \mathbf{T}-\mu_{\ln }}{\sigma_{\ln }}$ has a standard normal distribution $\Phi$ (with mean 0 and variance 1 ). Hence, if $\Psi$ is a sample from $\Phi$ and $\tau$ is the corresponding sample from $\mathbf{T}$, we have:

$$
\begin{aligned}
\Psi & =\frac{\ln \tau-\mu_{\ln }}{\sigma_{\ln }} \\
\tau & =\exp \left(\mu_{\ln }+\Psi \sigma_{\ln }\right)
\end{aligned}
$$

Given that the Time-To-Failure of an interconnect in a power grid is modeled with a lognormal distribution, a TTF sample from its distribution can be obtained using (2.29). The random samples $\Psi$ from $\Phi$ are obtained using the Zigguart method [32].

Eq (2.29) can be simplified further by using (2.26):

$$
\begin{aligned}
& \tau=\exp \left(\ln \left(\mu_{T}\right)-0.5 \sigma_{\ln }^{2}+\Psi \sigma_{\ln }\right) \\
& \tau=\mu_{T} \exp \left(\Psi \sigma_{\ln }-0.5 \sigma_{\ln }^{2}\right)
\end{aligned}
$$

Since $\sigma_{\ln }$ is constant, the TTF sample $\tau$ is directly proportional to the MTF $\mu_{\mathrm{T}}$, for a given $\Psi$.

\subsection{Power Grid Model}

A complete model of the power grid would include parasitic resistances, capacitances and (possibly) inductances, with the underlying logic blocks modeled as non-negative transient current sources connected between a subset of the grid nodes and ground. The transient current waveforms are assumed to be periodic. However, electromigration is a long term cumulative failure mechanism, and as such the transients in the currents that happen in the logic circuitry (at a scale of $\sim 10^{-9} s$ ) do not impact the degradation process significantly. The standard practice, as was mentioned before, is to use an effective-EM current to model the lifetimes of interconnects in the power grid, which is a DC value.

A power grid is a linear system, with the circuit currents acting as inputs and node 


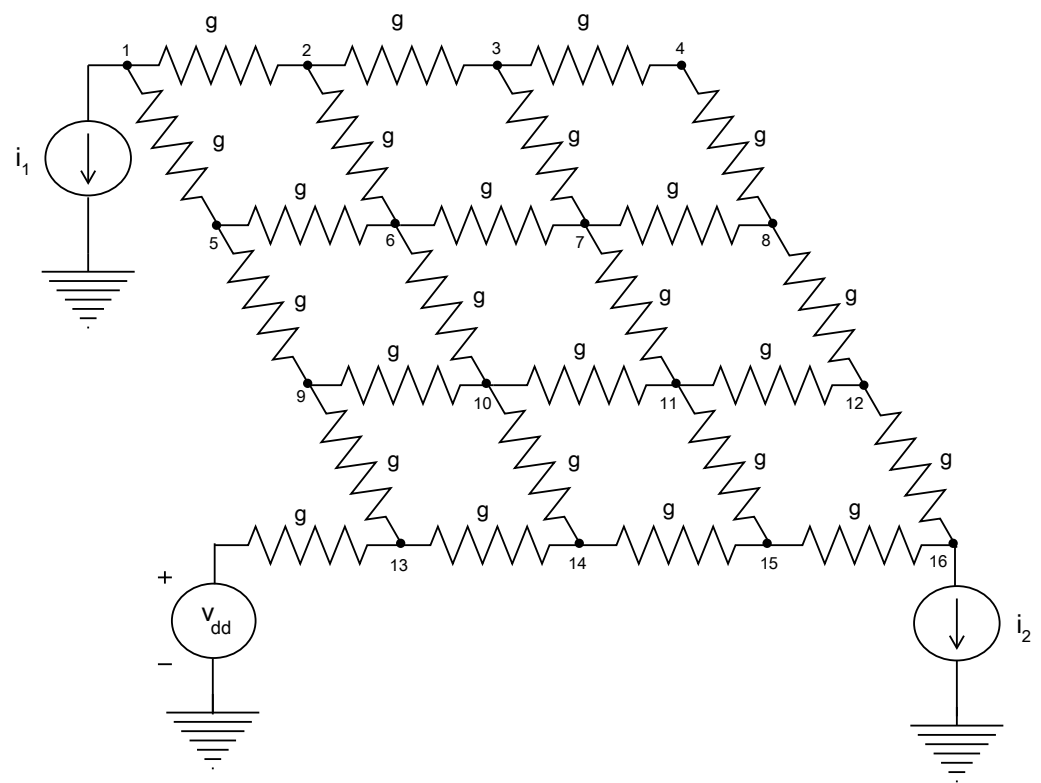

Figure 2.3: A typical DC model of power grid

voltage drops as the outputs:

$$
v(t)=\mathcal{L}[i(t)]
$$

where $\mathcal{L}$ is a linear operator representing the power grid. An interconnect in the power grid mostly carries uni-directional currents, so that its EM analysis (using Black's Equation) depends on the average current only. Because the power grid is a linear system, the average current in an interconnect can be obtained directly from the circuit current averages by doing a DC analysis. Also, the average output response of a linear system can be found by subjecting it to the average of its input stimulus (this can be easily proved using the superposition and the time-invariance property of a linear system). Hence, the grid node voltage drop averages can be obtained directly from the circuit current averages by means of a DC analysis:

$$
\langle v(t)\rangle=\mathcal{L}[\langle i(t)\rangle]=\mathcal{L}_{D C}[\langle i(t)\rangle]
$$

In Chapter 3, we will propose a framework for EM verification that depends on userprovided thresholds on the average voltage drops on the grid nodes. In this framework, it becomes sufficient therefore to perform DC analysis of the grid, driven by the averages of the circuit currents. Therefore, a DC model of the power grid, devoid of capacitances and inductances, is sufficient. Moreover, a DC model is highly computationally advantageous.

The power grid nodes are numbered $1,2 \ldots m$, with the ground node being 0 . Let $i$ be the vector of DC current stimuli. We assume that $i$ is well-defined for all nodes $1,2 \ldots m$, 
so that a node $k$ with no connected current source will have $i_{k}=0$. Let $u_{k}$ be the voltage at some node $k$, and $u(t)$ be the vector of all $u_{k}(t)$ signals. Applying Kirchoff's current law (KCL) at every node leads to the following matrix formulation:

$$
G(t) u(t)=-i+G_{v}(t) u_{d d}
$$

where $G(t)$ and $G_{v}(t)$ are $m \times m$ conductance matrices resulting from application of the traditional modified nodal analysis formulation [33]. In this case, $G(t)$ and $G_{v}(t)$ vary over large time-scales, as the lines age and deform, hence the time dependence. The matrix $G(t)$ is known to be a diagonally dominant symmetric positive definite $\mathcal{M}$-matrix (so that $G(t)^{-1} \geq 0$ ) [34]. $G_{v}(t)$ is a $m \times m$ matrix of conductance elements connected to $v_{d d}$ sources [35], and $u_{d d}$ is a constant vector each entry of which is equal to $v_{d d}$. Notice that if we set $i=0$, then obviously $u_{k}(t)=v_{d d} \forall t$, so that the above system becomes:

$$
G(t) u_{d d}=G_{v}(t) u_{d d}
$$

By replacing $G_{v}(t) u_{d d}$ by $G(t) u_{d d}$ in $(2.33)$, it can be re-written as:

$$
G(t)\left[u_{d d}-u(t)\right]=i
$$

We can express (2.35) in a more convenient way, directly in terms of voltage drops: define $v_{k}(t)=v_{d d}-u_{k}(t)$ to be the voltage drop at node $k$, and let $v(t)$ be the vector of voltage drops, then the system equation can be written as:

$$
G(t) v(t)=i
$$

This is a revised system equation which we can use directly to solve for voltage drop values. Comparing (2.36) to (2.33), it is easy to see that the circuit described by this equation consists of the original power grid, but with all voltage sources set to zero (short circuit) and all current source directions reversed.

\subsection{Monte Carlo random sampling approach}

Random sampling refers to iteratively selecting/generating sample values from the domain of a given distribution (according to their probabilities) and computing the arithmetic average of these samples as an estimate of the required quantity, a so-called Monte Carlo approach. The Monte Carlo approach can be used to estimate the mean of a distri- 
bution or the probability of occurrence of an event; we use it later to estimate the power grid MTF and survival probability. The key feature of the Monte Carlo approach, as we will shortly see, is that the desired accuracy (in terms of relative error) and confidence (a measure of how certain we are about the estimate) can be specified up-front by the user.

\subsubsection{Mean Estimation}

Estimating the mean of a distribution is a classic problem in statistics. In this subsection, we will review a standard approach employed to achieve the same. Let $\mathbf{X}_{\mathbf{1}}, \mathbf{X}_{\mathbf{2}} \ldots \mathbf{X}_{\mathbf{w}}$ be $w$ independent RVs that are identically distributed with the same distribution $f(x)$, that has a mean $\mu$ and variance $\sigma^{2} \geq 0$. Such a collection is called a random sample. The arithmetic average of this random sample is another RV $\overline{\mathbf{X}}_{\mathbf{w}}$ given by:

$$
\overline{\mathbf{X}}_{\mathrm{w}}=\frac{\mathbf{X}_{1}+\mathbf{X}_{2}+\ldots \mathbf{X}_{\mathbf{w}}}{w}
$$

It can be easily shown that mean of $E\left[\overline{\mathbf{X}}_{\mathbf{w}}\right]=\mu$ and $\operatorname{Var}\left(\overline{\mathbf{X}}_{\mathbf{w}}\right)=\sigma / w$. Thus, the distribution of $\overline{\mathbf{X}}_{\mathbf{w}}$ is narrower than $\mathbf{X}_{\mathbf{i}}$ and tightens around its mean as $w$ increases. In the limit, this is expressed as the law of large numbers:

$$
\lim _{w \rightarrow \infty} \overline{\mathbf{X}}_{\mathbf{w}}=\mu
$$

\section{Sampling from a Normal distribution}

Now, suppose that $\mathbf{X}$ has a normal distribution. In this case, it can be shown that $\overline{\mathbf{X}}_{\mathbf{w}}$ also has a normal distribution and $\mathbf{Z}=\frac{\overline{\mathbf{X}}_{\mathbf{w}}-\mu}{\sigma / \sqrt{w}}$ is an RV with standard normal distribution $\Phi$. For $\alpha \in[0,1]$, let $z_{\alpha / 2}$ be such that:

$$
\begin{aligned}
& \mathcal{P}\left\{\mathbf{Z}>z_{\alpha / 2}\right\}=1-\Phi\left(z_{\alpha / 2}\right)=\alpha / 2 \\
& \Phi\left(z_{\alpha / 2}\right)=\mathcal{P}\left\{\mathbf{Z} \leq z_{\alpha / 2}\right\}=1-\alpha / 2
\end{aligned}
$$

The value of $z_{\alpha / 2}$ for a given $\alpha$ can be easily found from statistical tables or using the $\operatorname{erf}()$ function on linux systems. Due to symmetry of the standard normal distribution 


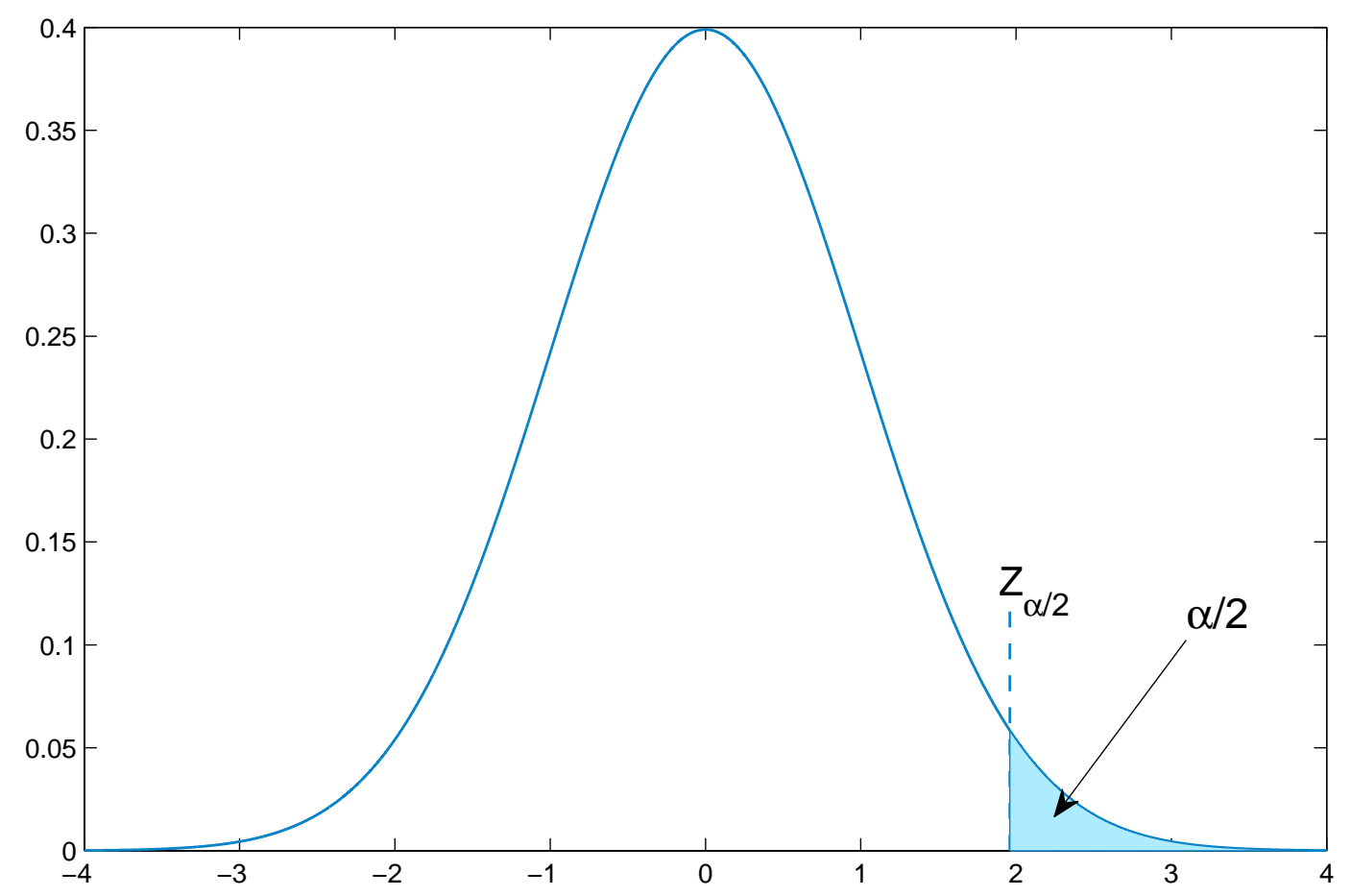

Figure 2.4: Definition of $z_{\alpha / 2}$

$\Phi$, it follows that

$$
\begin{array}{r}
\mathcal{P}\left\{-z_{\alpha / 2} \leq \mathbf{Z} \leq z_{\alpha / 2}\right\}=1-\alpha \\
\mathcal{P}\left\{-z_{\alpha / 2} \leq \frac{\overline{\mathbf{X}}_{\mathbf{w}}-\mu}{\sigma / \sqrt{w}} \leq z_{\alpha / 2}\right\}=1-\alpha \\
\mathcal{P}\left\{\overline{\mathbf{X}}_{\mathbf{w}}-z_{\alpha / 2} \frac{\sigma}{\sqrt{w}} \leq \mu \leq \overline{\mathbf{X}}_{\mathbf{w}}+z_{\alpha / 2} \frac{\sigma}{\sqrt{w}}\right\}=1-\alpha
\end{array}
$$

If we choose $\alpha=0.05$, then $Z_{0.025}=1.96$ and we have:

$$
\mathcal{P}\left\{\overline{\mathbf{X}}_{\mathbf{w}}-1.96 \frac{\sigma}{\sqrt{w}} \leq \mu \leq \overline{\mathbf{X}}_{\mathbf{w}}+1.96 \frac{\sigma}{\sqrt{w}}\right\}=0.95
$$

so that, if $\bar{X}_{w}$ is the observed value of $\overline{\mathbf{X}}_{\mathbf{w}}$ (i.e. mean of the observed sample values $X_{1}, X_{2} \ldots X_{w}$ ), we can assert with $95 \%$ confidence that the true mean $\mu$ lies within $1.96 \sigma / \sqrt{w}$ of the observed mean:

$$
\bar{X}_{w}-1.96 \frac{\sigma}{\sqrt{w}} \leq \mu \leq \bar{X}_{w}+1.96 \frac{\sigma}{\sqrt{w}}
$$




\section{Stopping Criterion when variance is known}

To derive a stopping criterion in terms of number of samples $w$ required, we need an upper bound on the relative error. From the previous analysis:

$$
\frac{\left|\bar{X}_{w}-\mu\right|}{\sigma / \sqrt{w}} \leq z_{\alpha / 2} \Longrightarrow \frac{\left|\bar{X}_{w}-\mu\right|}{\left|\bar{X}_{w}\right|} \leq \frac{z_{\alpha / 2} \sigma}{\left|\bar{X}_{w}\right| \sqrt{w}}
$$

If $w$ is large enough, so that

$$
\frac{z_{\alpha / 2} \sigma}{\left|\bar{X}_{w}\right| \sqrt{w}} \leq \delta
$$

for some $\delta \in(0,1)$, then $\delta$ places an upper bound on the relative error with $(1-\alpha) \times 100 \%$ confidence. Thus we have:

$$
\frac{\left|\bar{X}_{w}-\mu\right|}{\left|\bar{X}_{w}\right|} \leq \delta \quad \text { or } \quad \frac{\left|\bar{X}_{w}-\mu\right|}{|\mu|} \leq \frac{\delta}{1-\delta}=\epsilon
$$

where $0<\epsilon<1$ is a small positive number with $\delta=\epsilon /(1+\epsilon)$ and we assume that $\mu \neq 0$. The user specifies $\epsilon$, an upper bound on the relative error with respect to the true mean $\mu$. The stopping criterion can now be written as (from (2.44)):

$$
w \geq\left(\frac{z_{\alpha / 2} \sigma}{\left|\bar{X}_{w}\right| \epsilon /(1+\epsilon)}\right)^{2}
$$

\section{Stopping criterion when variance is unknown}

In most cases, the variance of the distribution will be unknown. Let $s_{w} \geq 0$ be the sample standard deviation, i.e.

$$
s_{w}^{2}=\frac{1}{w-1} \sum_{i=1}^{w}\left(X_{i}-\bar{X}_{w}\right)^{2}=\frac{w\left(\sum X_{i}^{2}\right)-\left(\sum X_{i}\right)^{2}}{w(w-1)}
$$

It can be shown that $s_{w}^{2}$ is an unbiased estimator of the variance. Then, the RV $\mathbf{t}=$ $\left[\left(\overline{\mathbf{X}}_{\mathbf{w}}-\mu\right) /\left(s_{w} / \sqrt{w}\right)\right]$ has Student's t-distribution with $(w-1)$ degrees of freedom. For $w \geq 30$, values of $t_{\alpha / 2}$ become very close to $z_{\alpha / 2}$, so that standard normal provides a good approximation to the t-distribution of size 30 or more [27]. Conservatively, we use $w \geq 50$ so that

$$
w \geq\left(\frac{z_{\alpha / 2} s_{w}}{\left|\bar{X}_{w}\right| \epsilon /(1+\epsilon)}\right)^{2} \quad \text { for } w \geq 50
$$




\section{Stopping criterion when not sampling from a Normal distribution}

In the preceding section, we derived the stopping criteria assuming a normal distribution for $\mathbf{X}$. However, in general, the distribution of $\mathbf{X}$ may not be normal. In such a case, we use the central limit theorem, which states that for large $w$, the $\operatorname{RV} \mathbf{Z}=\frac{\overline{\mathbf{X}}_{\mathbf{w}}-\mu}{\sigma / \sqrt{w}}$ approaches the standard normal distribution $\Phi$ (in practice, for $w$ as small as 25 or 30, distribution of $\mathbf{Z}$ approaches $\Phi)$. Further, studies [27] have shown that even when we replace $\sigma$ by its unbiased estimate $s_{w}$ (from (2.47)), $\mathbf{Z}$ is fairly close to a t-distribution provided $\mathbf{X}$ is not too skewed; and if it is, we can use a non-linear transform (such as exponential, logarithm etc.) to make the distribution more bell-shaped.

In conclusion, for most practical cases, we simply use (2.48) to determine the number of samples $w$ required in order to estimate the mean.

\subsubsection{Probability Estimation}

The Monte Carlo random sampling approach can also to be used to estimate the probability of occurrence of an event. Later in this work, we deal with the problem of estimating the survival probability of a power grid for a period of $y$ years as it undergoes degradation due to electromigration. Suppose there is a function that takes as input a power grid and generates a sample grid Time-to-Failure. In order to determine the required survival probability, Bernoulli trials are performed: $w$ grid TTF samples are generated using the given function. Generation of a sample TTF is referred as a trial. For each TTF sample, we have a success if the grid survives up to $y$ years, else we have a failure. If $p$ is the probability of a success, and if $x$ successes are observed in a sequence of $w$ trials, then by the law of large numbers [27]:

$$
\lim _{w \rightarrow \infty} \frac{x}{w}=p
$$

This would be a straightforward approach to determine the survival probability of a power grid, but it suffers from the defect that there is no way to tell when $x / w$ is close enough to $p$, for the process to be stopped. The solution for the same is derived in [36], and is based on statistical estimation of proportions. The stopping criterion is found to be:

$$
w=\max \left[\left(\frac{z_{\alpha / 2}}{2 \mathcal{E}}\right)^{2},\left(\frac{\sqrt{63}+z_{\alpha / 2}}{2 \sqrt{\mathcal{E}}}\right)^{2},\left(\frac{z_{\alpha / 2} \sqrt{2 \mathcal{E}+0.1}+\sqrt{(\mathcal{E}+0.1) z_{\alpha / 2}^{2}+3 \mathcal{E}}}{2 \mathcal{E}}\right)^{2}\right]
$$



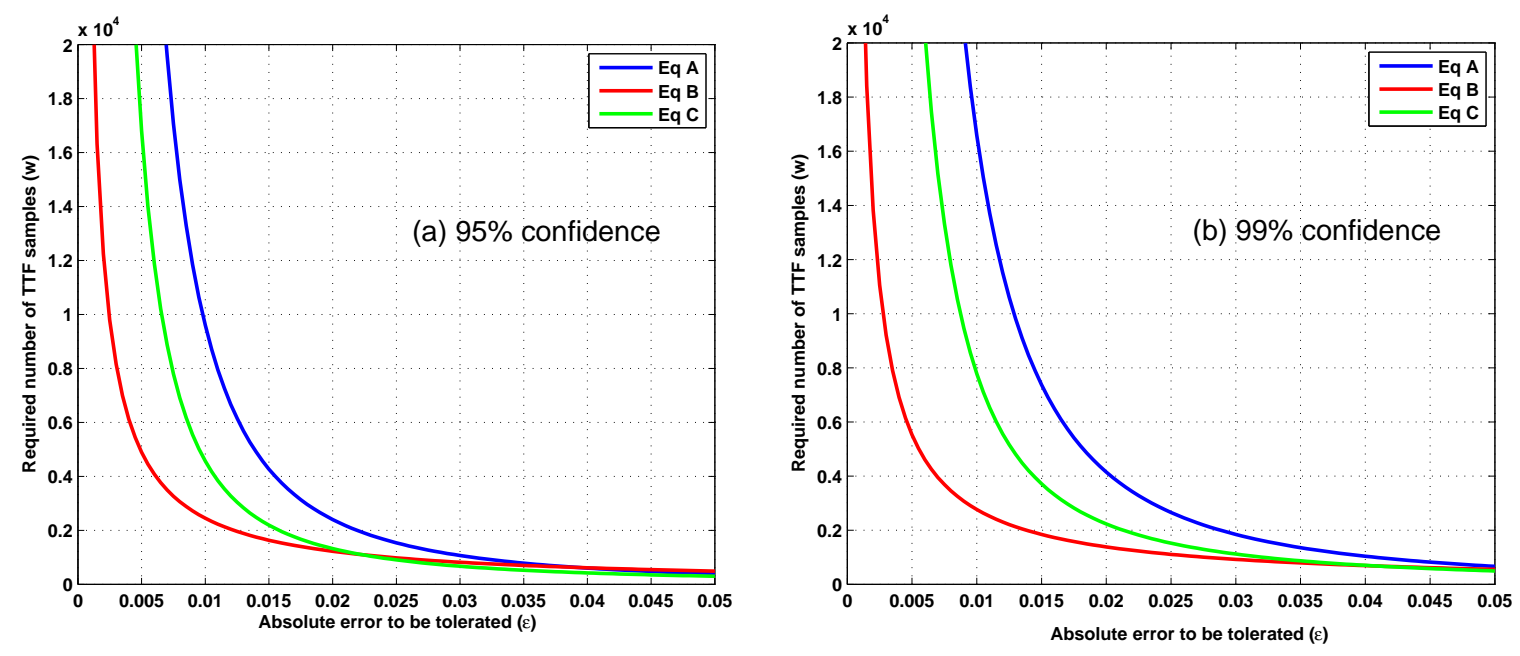

Figure 2.5: Comparison of Statistical Bounds for 95\% and 99\% confidence, where Eq. A: $\left(z_{\alpha / 2} /(2 \mathcal{E})\right)^{2}$, Eq. B: $\left(\left(\sqrt{63}+z_{\alpha / 2}\right) /(2 \sqrt{\mathcal{E}})\right)^{2}$ and

Eq C: $\left(\left(z_{\alpha / 2} \sqrt{2 \mathcal{E}+0.1}+\sqrt{(\mathcal{E}+0.1) z_{\alpha / 2}^{2}+3 \mathcal{E}}\right) /(2 \mathcal{E})\right)^{2}$

where $\alpha$ and $\mathcal{E}$ are small numbers between 0 and 1. $\alpha$ determines the confidence level as to how closely $x / w$ approximates $p$, whereas $\mathcal{E}$ is maximum the absolute error in estimation, i.e. the trials are stopped when "there is $(1-\alpha) \times 100 \%$ confidence that $\left|\frac{x}{w}-p\right|<\mathcal{E}^{\prime}$.

The major difference between mean estimation and probability estimation is that in probability estimation, the number of samples required can be pre-determined for a given $\alpha$ and $\mathcal{E}$. However, in mean estimation, the stopping criterion depends on the coefficient of variation of obtained samples $\left(s_{w} /\left|\bar{X}_{w}\right|\right)$ during the Monte Carlo experiment. Hence, the number of samples required for mean estimation cannot be pre-determined.

\subsection{Electromigration checking in power grids}

Many empirical and analytical models have been proposed to explain degradation due to EM in a metal line [5][37][9]. All these models represent the atomic flux divergence as some function of the current density in the line. This lead to the use of current density limits for individual interconnects to arrive at reliable designs for ICs, the idea being that if each wire is made safe from EM degradation by keeping its current density below the design limit, the chip is guaranteed to be safe. The design limits were based on 'worst case' estimates of the current density that is expected under use conditions, usually maximum temperature [38]. The designers used a very simple criterion for elec- 


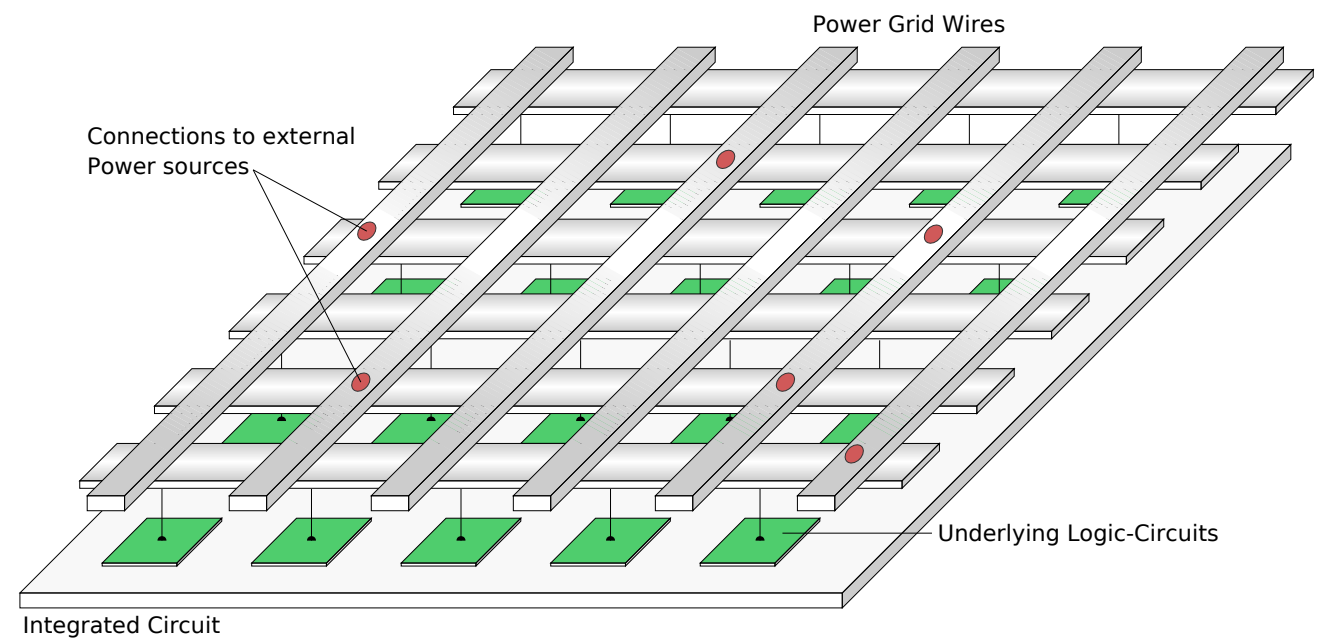

Figure 2.6: A typical on-die modern power grid with a mesh structure

tromigration checking, they compared interconnect dc effective current per unit width, $i_{e f f}$, to a conservative fixed limit:

$$
\mathcal{S}=\frac{\text { Actual } i_{e f f}}{\text { Design Limit } i_{e f f}}
$$

A reliable design with regards to electromigration was obtained when $\mathcal{S}<1$, and any interconnect with $\mathcal{S}>1$ was re-designed. However, this approach was very restrictive, because it is highly improbable that all the lines in power grids will carry the 'design limit $i_{\text {eff }}$ ' currents at the same time. Even if it happens, a chip dissipating power in kilowatts will be required, which is infeasible.

As the designs became more complex, there was a need to move away from this highly restrictive and pessimistic EM analysis and to elevate electromigration checking to the realm of full-chip analysis. Models and methods, such as those in BERT [39], were developed to estimate damage due to EM, but they were tested only on small test circuits. Frost and Poole [3] suggested a series model to determine the reliability of an integrated circuit. Under a series model assumption, a system is said to have failed as soon as any of its components fails. However, it was pointed out later [4] that when the total statistical risk of the chip is of concern, the explicit limit placed on each and every interconnect becomes arbitrary; it is not necessary to guarantee that each and every interconnect should have $\mathcal{S}<1$ for the chip to be safe. As per the series model (with some simplifying assumptions), the failure rate of the system is the sum of failure rates of individual components. This allowed budgeting the chip level reliability among the 
interconnects, thus minimizing to an extent the performance limitations imposed by EM reliability goal in the design. The series model with budgeting was applied to the Alpha 21164 microprocessor, under the name Statistical Electromigration Budgeting (SEB) [4] and became a standard technique for EM checking in many industrial CAD tools. [38] also used the series system model and 'reliability budgeting' to perform full chip power grid and signal net electromigration and joule heating analysis.

Though the power grid lines are usually wider than the signal lines, they are more susceptible to EM failures because they carry uni-directional currents and thus do not experience the benefit of healing [40][41]. Added to that, the series system assumption, though valid for signal lines (which have no redundancy, except for redundant vias), is highly pessimistic for modern mesh power grids. A typical mesh power grid is shown in figure 2.6. In this work, we overcome this limitation by accounting for redundancy while estimating the failure statistics of the power grid. 


\section{Chapter 3}

\section{Mesh model for Power Grid Reliability Verification}

\subsection{Introduction}

In this chapter, we propose a new model for on-die power grid reliability verification under the influence of electromigration. The new model takes into account the inherent redundancy of the power grid structure while estimating it's Mean Time to Failure (MTF) and reliability, which is completely ignored by the present state-of-the-art EM checking tools. This new model is referred as the mesh model. Among other things, an implementation of the mesh model requires us to update the node voltage drops as the power grid suffers damage due to electromigration. To do the same, we also describe a highly efficient exact method based on Woodbury formula and Banachiewicz-Schur form to update the solution of a linear system $G v=i$ as $G$ undergoes low rank updates.

The chapter is organized as follows. Section 3.2 introduces a simple and conservative open-circuit failure model for an interconnect in the power grid. In section 3.3, we describe a new criteria to determine power grid failure, that accounts for the redundancies in the mesh structure. Next, the mesh model is presented in detail, followed by the proposed method to efficiently update the voltage drops. The implementation and results in this chapter focus on the speed-up obtained in updating voltage drops by using Woodbury formula and Banachiewicz-Schur form over Sparse-LU. 


\subsection{Failure model for a single interconnect}

Due to high current densities, the resistance of interconnects in the power grid changes as they undergo degradation due to EM. Resistance evolution of a typical interconnect is shown in figure 3.1; it remains almost equal to the initial value up to some time $t=\tau$ (while the void nucleates and grows), after which it experiences a step increase in its resistance value (when the void spans the entire cross-section) followed by a steeplinear increase (corresponding to the longitudinal void growth) [42]. This is referred to as the step-increase linear model of EM degradation in an interconnect. Failure of an interconnect in this model is based on a percentage increase in resistance value, which usually happens around $t=\tau$.

The step-increase linear model, though accurate, is hard to simulate due to it's nonlinearity. This work instead proposes a simple and intuitive model for degradation due to EM, the open-circuit model. As the name suggests, the failure of an interconnect in this model is defined to be an open-circuit. Thus, at $t=\tau$, the interconnect is effectively removed from the power grid. The reader should note that the open-circuit model is conservative as compared to the step-increase linear model (see figure 3.2). The former model assumes an interconnect to have failed once its resistance has risen above some threshold (derived from the percentage increase). Hence, lines continue to conduct current, albeit with high resistance, after the time-to-failure $(t=\tau)$ predicted by EM models. By assuming zero conductance after the predicted TTF, the open-circuit model is thus conservative.

\subsection{New criteria for power grid failure}

Due to scaling, supply voltages in deep sub-micron CMOS are reducing. With a reduced $v_{d d}$, even a small drop in the local supply voltage can have a significant effect on the circuit timing. As was shown in [43], if the supply voltage varies up to $12.5 \%$, one can observe (by simulation) up to $2.4 X$ increase in gate delay. Thus, reliable functioning of a chip is tightly coupled to the issue of voltage drop in its power supply network. Since the voltage drop of a node directly affects the delay of the underlying logic, it then clearly implies that the underlying logic will fail if the voltage drop exceeds a certain threshold value. In other words, if the node voltage drops exceed the budget, speed-critical devices will be slowed and the chip will not meet its intended performance goal. As such, a node is deemed to be safe only when its voltage drop is below the threshold, otherwise it is said to have failed. 


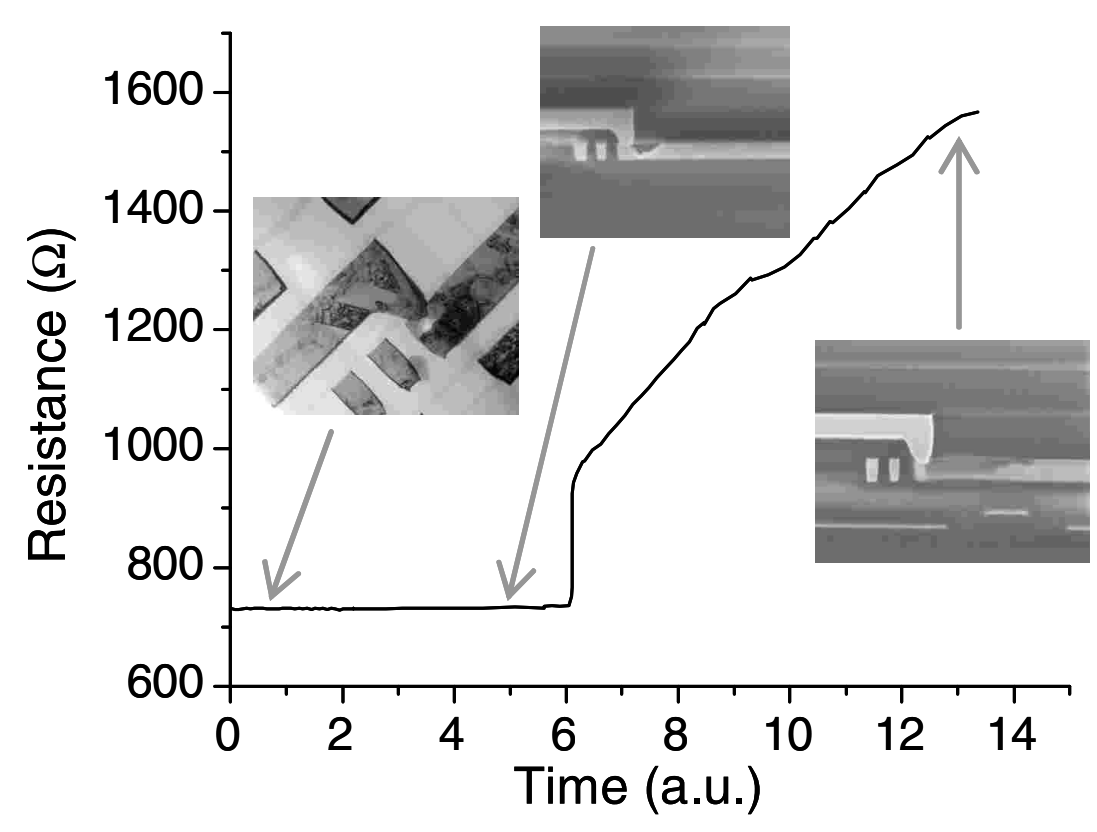

Figure 3.1: Resistance and void shape evolution with time [42]

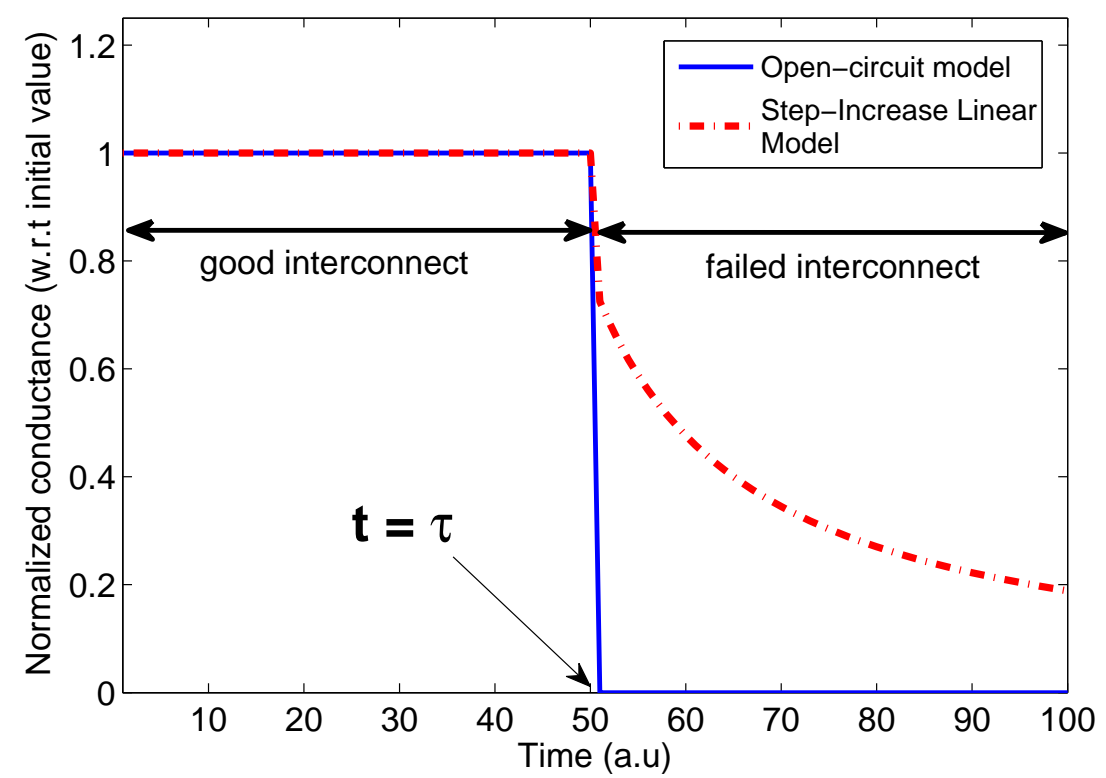

Figure 3.2: Conductance change with EM degradation for the step-increase linear model and open-circuit model 
A power grid is functional as long as it can ensure the reliable functioning of the underlying logic. Such a power grid is said to be safe. From the previous paragraph, one can easily see that the safety of the power grid is tied to the safety of its nodes. Thus, the new failure criteria is:

A power grid is said to be safe iff all the nodes are safe, otherwise it is deemed to have failed.

Typically, a chip is designed with an expected voltage drop budget of between $5 \%$ and $10 \%$ of $v_{d d}$. The simulation and timing verification are performed while accounting for this budget [44]. Hence, for this work, the voltage drop threshold was defined to be $10 \%$ of $v_{d d}$ for all nodes in the grid. The new failure criteria automatically captures the redundancies in the grid, because the voltage drop of a node is determined from the grid topology itself. As opposed to the series model, the voltage drop threshold based failure criteria allows the power grid to tolerate many interconnect failures before it is declared failed.

\subsection{New grid failure model: Mesh model}

As mentioned before, for the purpose of EM reliability verification, the power grid is modeled as a resistive mesh, with the underlying logic blocks being represented by the corresponding circuit current averages connected between a subset of grid nodes and ground. Any variation of source currents due to voltage drops is ignored. As per the new failure criteria, a voltage-drop threshold value for every grid node (or a subset of grid nodes) is given, and is captured in the $m \times 1$ vector $v_{t h}$. Note that $v_{t h}$ is a user-provided threshold on the average voltage drops on the grid. To avoid trivial cases, it is assumed that $i \neq 0$ and $v_{t h}>0$.

At $t=0$, the fresh power grid satisfies the following conditions:

1. The grid is connected, so that there is a resistive path from any node to any other node that does not go through a $v_{d d}$ supply node or a ground node.

2. The voltage drops on all the nodes of the power grid are below the specified thresholds, i.e., if $v_{0}=v(0)$, then we have $v_{0}<v_{t h}$.

These conditions ensure that the power grid is safe at $t=0$.

Since EM is not a deterministic process, there are many possible sequences in which the interconnects could fail. Each sequence of interconnect failure that leads to a grid 


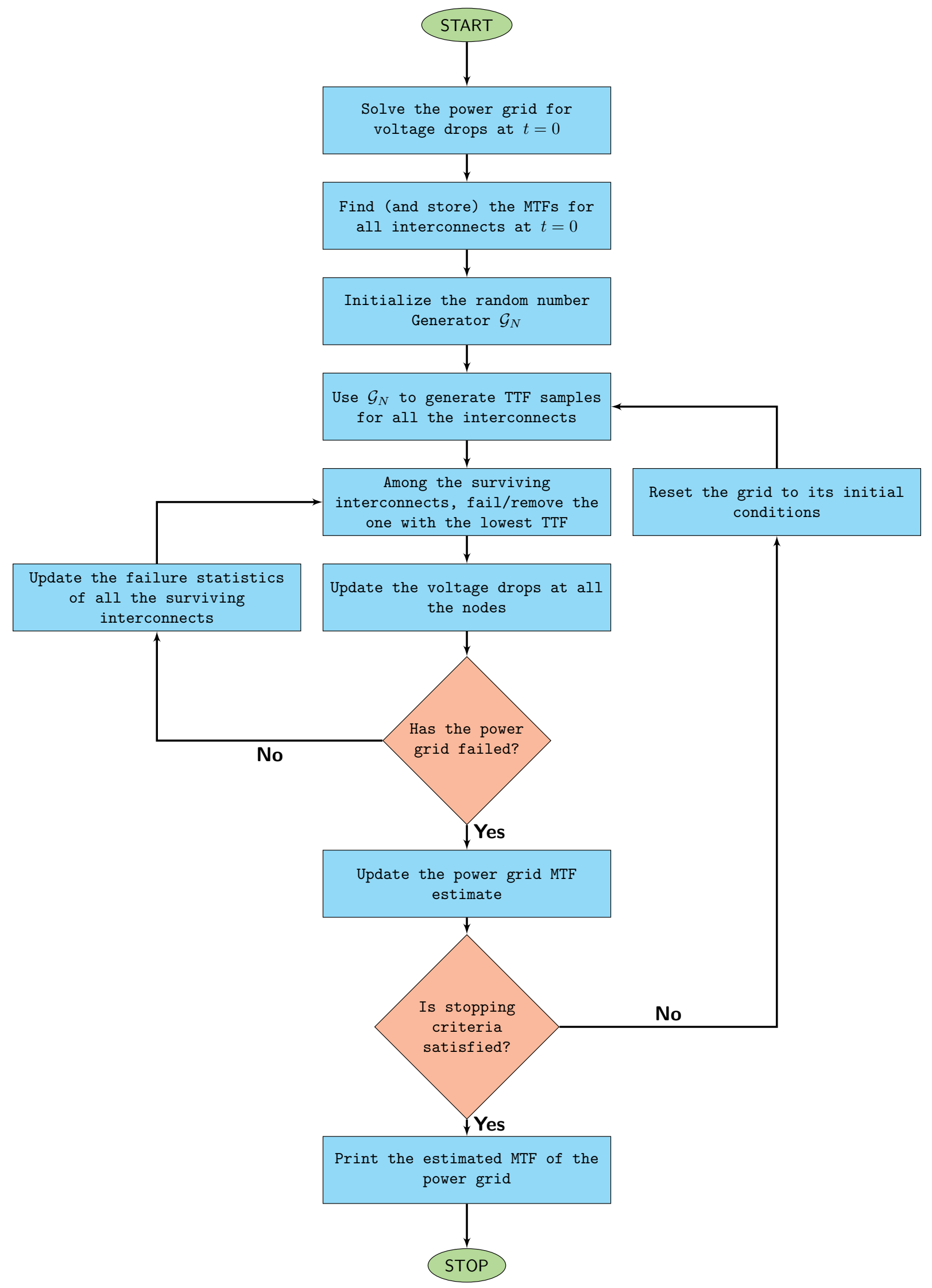

Figure 3.3: Flow-chart for the mesh model 
failure, is called a realization. In any particular realization, the interconnects have assigned sample TTF values from their respective lognormal distributions. For a given realization, as time progresses the grid lines start to deform and fail due to electromigration (in increasing order of TTF samples). As per the open-circuit model, every failure corresponds to removal of an interconnect from the grid. Thus, $\|G(t)\|$ decreases with time, which leads to an increase in $\|v(t)\|$ :

$$
\|G(t)\|\|v(t)\| \geq\|G(t) v(t)\|=\|i\| \Longrightarrow\|v(t)\| \geq \frac{\|i\|}{\|G(t)\|}
$$

In other words, as interconnects fail, the overall voltage drop on the nodes of the grid increases. Also, removing interconnects from the grid increases grid sparsity. Given that the vector of source currents $i$ is constant, the surviving interconnects (on an average) should conduct higher currents as time progresses, which makes them more susceptible to failure due to EM. Hence, the failure statistics of the surviving lines are dependent on the previous failures. As such, the interconnects are not statistically independent in the mesh model. Thus, until the grid fails, there is a need to update the following after each interconnect failure: i) the voltage drops at the nodes of the grid and ii) the failure statistics of the surviving interconnects.

The grid is deemed to fail at the earliest time for which the condition $v(t) \leq v_{t h}$ is no longer true, which can happen either due to voltage drop(s) at some node(s) exceeding their threshold value or due to a singular grid (i.e. as the resistors are removed, a node becomes completely disconnected from the other nodes, causing $\operatorname{det}(G(t))=0)$. A singular grid models the scenario where all resistors connected to a particular node have higher than threshold resistance due to EM. As a conservative approximation this node is assumed to have failed, causing the grid to fail. Once a grid has failed, it is assumed that it remains failed for all the future time. This model, used to determine the Time-to-Failure (TTF) of the power grid, is henceforth referred to as the mesh model.

In order to estimate the MTF and reliability of the power grid using the mesh model, Monte Carlo random sampling approach is employed: we simulate different realizations using the mesh model and obtain the corresponding grid TTF samples until the stopping criteria (in terms of number of grid TTF samples required) as derived in (2.48) is satisfied. The overall flow of the mesh model is shown in the flowchart of figure 3.3. 


\subsection{Efficiently updating Voltage Drops}

As noted, in order to implement the mesh model, there is a need to update the voltage drop of the nodes and the failure statistics of the surviving interconnects after each interconnect failure. The problem of updating the failure statistics of the surviving lines is presented in Chapter 4. In this section, we describe a highly efficient approach for updating the node voltage drops in the power grid.

\subsubsection{Problem definition}

In order to update the voltage drops efficiently, observe that the conductance matrix $G$ changes by $\Delta G_{k}$ after the $k^{\text {th }}$ interconnect fails:

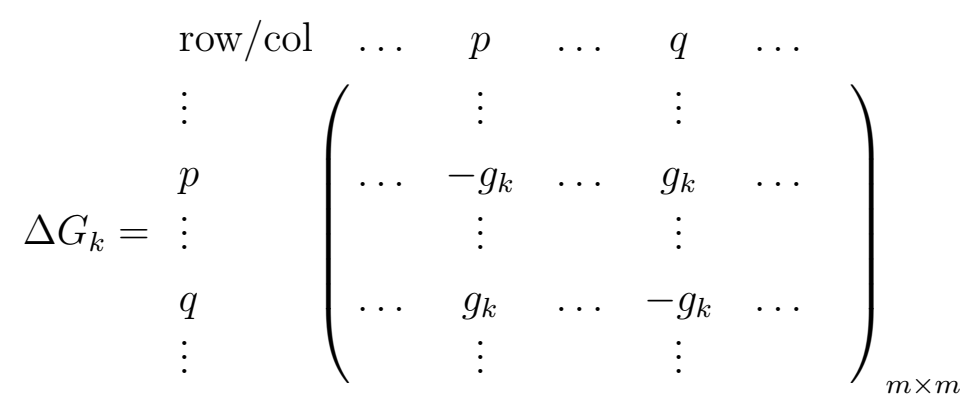

Note that $-\Delta G_{k}$ is the $m \times m$ conductance stamp of the $k^{t h}$ interconnect in the MNA matrix [45]. $g_{k}$ is the conductance of the $k^{\text {th }}$ failed interconnect, connected between nodes $p$ and $q$ with $p<q$.

Proposition 1. $\Delta G_{k}$, as defined in (3.2), is a rank-1 matrix.

Proof. A rank-1 matrix has only one linearly independent column. In other words, if $\Delta G_{k}$ is a rank-1 matrix, all columns of $\Delta G_{k}$ can be expressed as a linear combination of (only) one column in $\Delta G_{k}$. Now, there are three unique columns in $\Delta G_{k}$ :

$$
\begin{aligned}
C_{0}^{T} & =\left[\begin{array}{lllll}
0 & \ldots & 0 & \ldots & 0
\end{array}\right]_{1 \times m} \\
C_{1}^{T} & =\left[\begin{array}{llllll}
\ldots & g_{k} & \ldots & -g_{k} & \ldots
\end{array}\right]_{1 \times m} \\
C_{2}^{T} & =\left[\begin{array}{llllll}
\ldots & -g_{k} & \ldots & g_{k} & \ldots
\end{array}\right]_{1 \times m}
\end{aligned}
$$

Clearly, $C_{0}=0 \times C_{1}$ and $C_{2}=-1 \times C_{1}$ so that all columns of $\Delta G_{k}$ can be expressed in terms of $C_{1}$. Hence, proved.

Thus, the problem of finding the new voltage drops as the interconnects are removed from the power grid can be stated as follows: Let $G_{0}$ be the conductance matrix at $t=0$. 
Let $\Delta G_{1}, \Delta G_{2}, \ldots, \Delta G_{k}$ be the rank-1 conductance stamps of the failed interconnects and $v_{0}, v_{1}, v_{2}, \ldots, v_{k}$ be the corresponding vectors of voltage drops such that:

$$
\begin{aligned}
G_{0} v_{0} & =i \\
\left(G_{0}+\Delta G_{1}\right) v_{1} & =i \\
\left(G_{0}+\Delta G_{1}+\Delta G_{2}\right) v_{2} & =i \\
& \vdots \\
\left(G_{0}+\Delta G_{1}+\Delta G_{2}+\ldots+\Delta G_{k}\right) v_{k} & =i
\end{aligned}
$$

It is required to find all the solution vectors $v_{0}, v_{1}, v_{2}, \ldots, v_{k}$ in an efficient way by taking advantage of the properties of $G_{0}$ and the low rank updates performed on it.

\subsubsection{Woodbury Formula for voltage updates}

Any $m \times m$ rank- 1 matrix can be written as the outer product of two column vectors of size $m$ [46]. Specifically,

$$
\Delta G_{k}=u_{k} h_{k}^{T} \text { such that } u_{k}=-h_{k}=\sqrt{g_{k}}\left(e_{q}-e_{p}\right)
$$

where $g_{k}$ is the conductance of the $k^{t h}$ failed interconnect, connected between nodes $p$ and $q$ with $p<q$ and $e_{\lambda}$ is a column vector of size $m$ containing 1 at the $\lambda^{\text {th }}$ location and zeros at all other locations, with $e_{0}$ being a vector of all zeros. Define $U$ and $H$ so that after $k$ failures:

$$
U \triangleq\left[\begin{array}{cccc}
\mid & \mid & & \mid \\
u_{1} & u_{2} & \ldots & u_{k} \\
\mid & \mid & & \mid
\end{array}\right] \text { and } H \triangleq-U
$$

Clearly, $\sum_{j=1}^{k} \Delta G_{j}=U H^{T}$. Thus, the vector of voltage drops $v_{k}$ after $k$ interconnect failures can be written as:

$$
v_{k}=\left(G_{0}+\sum_{j=1}^{k} \Delta G_{j}\right)^{-1} i=\left(G_{0}+U H^{T}\right)^{-1} i
$$

Now, the Woodbury formula gives [47]:

$$
\left(G_{0}+U H^{T}\right)^{-1}=G_{0}^{-1}-\left[G_{0}^{-1} U\left(I_{k}+H^{T} G_{0}^{-1} U\right)^{-1} H^{T} G_{0}^{-1}\right]
$$


where $I_{k}$ is $k \times k$ identity matrix. Using (3.6) in (3.5), we have:

$$
v_{k}=G_{0}^{-1} i-\left[G_{0}^{-1} U\left(I_{k}+H^{T} G_{0}^{-1} U\right)^{-1} H^{T} G_{0}^{-1}\right] i
$$

We know that $G_{0}^{-1} i=v_{0}$, where $v_{0}$ is the voltage drop vector at $t=0$. Define

$$
Z \triangleq G_{0}^{-1} U=\left[\begin{array}{lll}
G_{0}^{-1} u_{1} \quad \ldots \quad G_{0}^{-1} u_{k}
\end{array}\right]
$$

Clearly, both $v_{0}$ and $Z$ can be efficiently found using one sparse $L U$ factorization of $G_{0}$ (into triangular matrices $L_{G}$ and $U_{G}$ ) and $k+1$ forward/backward substitutions. Hence, (3.7) can be re-written as:

$$
v_{k}=v_{0}-Z\left(I_{k}+H^{T} Z\right)^{-1} H^{T} v_{0}
$$

Let $W_{k} \triangleq\left(I_{k}+H^{T} Z\right)$ and $y_{k} \triangleq H^{T} v_{0}$. Eq. (3.9) becomes:

$$
v_{k}=v_{0}-Z W_{k}^{-1} y_{k}
$$

Given an initial LU factorization of $G_{0}$, updating the voltage drops using (3.10) will require one backward/forward substitution $\left(\mathcal{O}\left(m^{2}\right)\right)$, one LU solve of a dense $k \times k$ system $\left(\mathcal{O}\left(k^{3}\right)\right)$, matrix-vector product $(\mathcal{O}(m k))$ and a vector-vector subtraction $(\mathcal{O}(m))$. Thus, the overall scalability of the updates is dominated by $\mathcal{O}\left(m^{2}\right)$ as long as $k<<m$. Hence, by using the woodbury formula, the costly step of factoring the conductance matrix after each interconnect failure has been eliminated.

Using Woodbury formula (3.10) to update the voltage drops has two key advantages: i) Even though the matrix $W_{k}$ is dense, the number of interconnects $k$ required to fail a grid is a very small fraction of the number of nodes $m$, hence finding $W_{k}^{-1} y_{k}$ by LU factorization is comparatively very cheap. ii) The matrices $Z$ and $H^{T} Z$ need not be calculated from scratch for each failure, but can be efficiently updated by appending appropriate vectors at the end.

However, for large grids, the number of interconnect failures required to fail a grid can be quite large. Solving a dense linear system $W_{k}^{-1} y_{k}$ using $L U$ factorization has $\mathcal{O}\left(k^{3}\right)$ complexity. In effect, this means that as $k$ increases, the voltage updates using woodbury formula slows down (though it is still faster than a complete $L U$ solve). To overcome this limitation, a further refinement based on Banachiewicz-Schur form is proposed so that the complexity of solving the linear system $W_{k}^{-1} y_{k}$ is reduced to $\mathcal{O}\left(k^{2}\right)$. 


\subsubsection{Improving scalability using Banachiewicz-schur form}

The matrix $W_{k}$ can be written as:

$$
\begin{aligned}
W_{k}= & I_{k}+H^{T} Z=I_{k}-U^{T} G_{0}^{-1} U=I_{k}-\left[u_{1} \ldots u_{k}\right]^{T}\left[G_{0}^{-1} u_{1} \ldots G_{0}^{-1} u_{k}\right] \\
= & {\left[\begin{array}{cccc}
1-u_{1}^{T} G_{0}^{-1} u_{1} & -u_{1}^{T} G_{0}^{-1} u_{2} & \ldots & -u_{1}^{T} G_{0}^{-1} u_{k} \\
-u_{2}^{T} G_{0}^{-1} u_{1} & 1-u_{2}^{T} G_{0}^{-1} u_{2} & \ldots & -u_{2}^{T} G_{0}^{-1} u_{k} \\
\vdots & \vdots & \ddots & \vdots \\
-u_{k}^{T} G_{0}^{-1} u_{1} & -u_{k}^{T} G_{0}^{-1} u_{2} & \ldots & 1-u_{k}^{T} G_{0}^{-1} u_{k}
\end{array}\right] }
\end{aligned}
$$

Since $G_{0}^{-1}$ is symmetric, then $u_{i}^{T} G_{0}^{-1} u_{j}=u_{j}^{T} G_{0}^{-1} u_{i} \forall i, j$. Hence, $W_{k}$ is symmetric and $W_{k}$ can be re-written in terms of $W_{k-1}$ as:

$$
W_{k}=\left[\begin{array}{cc}
W_{k-1} & b_{k} \\
b_{k}^{T} & d_{k}
\end{array}\right]
$$

such that

$$
\begin{aligned}
b_{k}=\left[\begin{array}{lll}
-u_{1}^{T} G_{0}^{-1} u_{k} & \ldots & -u_{k-1}^{T} G_{0}^{-1} u_{k}
\end{array}\right]^{T} \in \mathbb{R}^{k-1} \\
d_{k}=1-u_{k}^{T} G_{0}^{-1} u_{k} \in \mathbb{R}
\end{aligned}
$$

Hence, using the Banachiewicz-schur form, $W_{k}^{-1}$ can be expressed in terms of $W_{k-1}^{-1}$ as (see Appendix):

$$
W_{k}^{-1}=\left[\begin{array}{cc}
W_{k-1}^{-1}+\frac{W_{k-1}^{-1} b_{k} b_{k}^{T} W_{k-1}^{-1}}{s_{k}} & \frac{-W_{k-1}^{-1} b_{k}}{s_{k}} \\
\frac{-b_{k}^{T} W_{k-1}^{-1}}{s_{k}} & \frac{1}{s_{k}}
\end{array}\right]
$$

where $s_{k}$ is the Schur complement of $W_{k-1}$ in $W_{k}$ and can be found using:

$$
s_{k}=d_{k}-b_{k}^{T} W_{k-1}^{-1} b_{k}
$$

Also, after $k$ interconnect failures, $y_{k-1}$ can be updated to $y_{k}$ by appending $p_{k} \triangleq-u_{k}^{T} v_{0}$ at the end:

$$
\begin{aligned}
& y_{k}=H^{T} v_{0}=-U^{T} v_{0}=-\left[\begin{array}{lll}
u_{1} & \ldots & u_{k}
\end{array}\right]^{T} v_{0}=-\left[\begin{array}{lll}
u_{1}^{T} v_{0} & \ldots & u_{k}^{T} v_{0}
\end{array}\right]^{T} \\
& =\left[\begin{array}{ll}
y_{k-1}^{T} & p_{k}
\end{array}\right]^{T}
\end{aligned}
$$


Then, $W_{k}^{-1} y_{k}$ can be written as:

$$
\begin{aligned}
& W_{k}^{-1} y_{k}=\left[\begin{array}{cc}
W_{k-1}^{-1}+\frac{W_{k-1}^{-1} b_{k} b_{k}^{T} W_{k-1}^{-1}}{s_{k}} & -\frac{W_{k-1}^{-1} b_{k}}{s_{k}} \\
-\frac{b_{k}^{T} W_{k-1}^{-1}}{s_{k}} & \frac{1}{s_{k}}
\end{array}\right]\left[\begin{array}{c}
y_{k-1} \\
p_{k}
\end{array}\right] \\
& =\left[\begin{array}{c}
W_{k-1}^{-1} y_{k-1}+\frac{W_{k-1}^{-1} b_{k} b_{k}^{T} W_{k-1}^{-1} y_{k-1}}{s_{k}}-\frac{W_{k-1}^{-1} b_{k}}{s_{k}} p_{k} \\
-\frac{b_{k}^{T} W_{k-1}^{-1} y_{k-1}}{s_{k}}+\frac{p_{k}}{s_{k}}
\end{array}\right]
\end{aligned}
$$

But, the previous solution $\gamma_{k-1} \triangleq W_{k-1}^{-1} y_{k-1}$ is known, therefore:

$$
\gamma_{k}=\left[\begin{array}{c}
\gamma_{k-1}+\frac{W_{k-1}^{-1} b_{k} b_{k}^{T} \gamma_{k-1}}{s_{k}}-\frac{W_{k-1}^{-1} b_{k}}{s_{k}} p_{k} \\
-\frac{b_{k}^{T} \gamma_{k-1}}{s_{k}}+\frac{p_{k}}{s_{k}}
\end{array}\right]
$$

Define $a_{k}=\frac{b_{k}^{T} \gamma_{k-1}-p_{k}}{s_{k}}$. Now, rewrite (3.18) as:

$$
\gamma_{k}=\left[\begin{array}{c}
\gamma_{k-1}+a_{k} W_{k-1}^{-1} b_{k} \\
-a_{k}
\end{array}\right]
$$

Hence, (3.14) and (3.19) can be used to directly update $W_{k}^{-1}$ and $\gamma_{k}$ from their previous values. Notice that $W_{k}^{-1}$ is required because, after the next interconnect failure, $W_{k}^{-1} b_{k+1}$ is needed to compute $\gamma_{k+1}$ using (3.19). The implementation requires a single matrixvector product $\left(\mathcal{O}\left(k^{2}\right)\right)$ and $\mathcal{O}\left(k^{2}\right)$ additions and divisions. Hence, the complexity of solving $W_{k}^{-1} y_{k}$ is reduced to $\mathcal{O}\left(k^{2}\right)$. This reduction in complexity comes into play only when $k$ is high enough.

Algorithm 1 computes the new voltage drops after the $k^{\text {th }}$ interconnect fails. It also sets the grid_singular flag if the last interconnect failure made the $G$ matrix singular. When $k=1, \gamma$ is easily calculated by a scalar division. For subsequent calls, $\gamma$ is updated from it previous solution using (3.19). Note that algorithm 1 implicitly receives the sequence of previous failures from matrices $Z$ and $H$. 


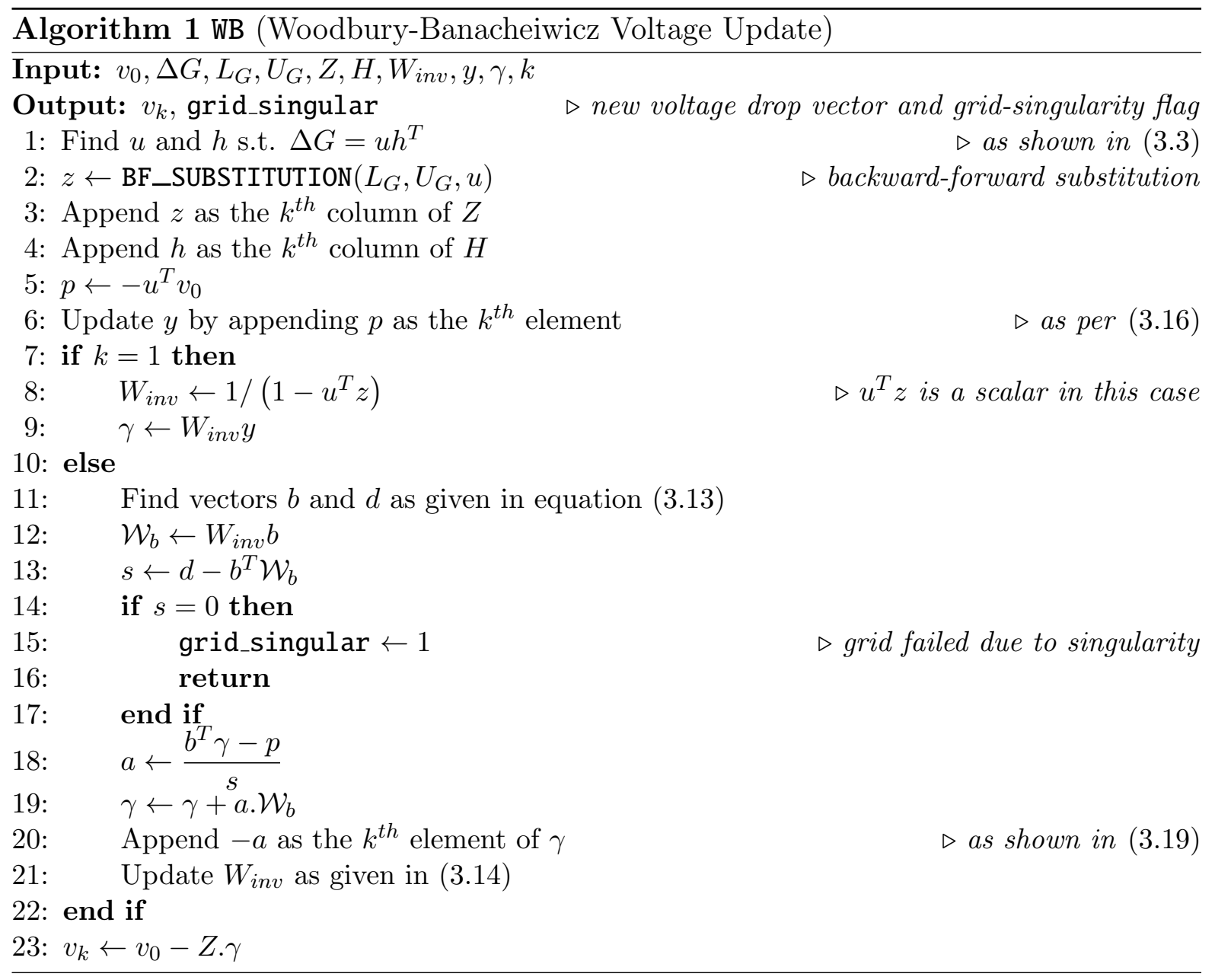

\subsection{Experimental Results}

A C++ implementation was written to test the speedup obtained by using the WoodburyBanachiewicz-Schur form over Sparse-LU (using UMFPACK) for updating the node voltage drops of the grid as the interconnects fail. UMFPACK [48] [49][50][51] is used to perform the initial LU factorization required for implementing Woodbury-BanachiewiczSchur form. Two types of test grids were used. The first type were generated per user specifications, including grid dimensions, metal layers, pitch and width per layer. The supply voltages and current sources were randomly placed on the grid. The technology specifications were consistent with $1.1 \mathrm{~V}$ 65nm CMOS technology. These grids are henceforth referred to as internal grids. The second type of grids are part of the IBM power grid benchmarks [52]. These grids are dual grids (i.e. they have both $v_{d d}$ and gnd rails of the power grid), but the proposed approach was tested only for $v_{d d}$ part of the grids and are referred to as external grids. Since the problem size is determined only by the node count $m$, other grid details are not given here. 


\begin{tabular}{|c|c|c|c|c|c|c|}
\hline \multicolumn{2}{|c|}{$\begin{array}{l}\text { Power } \\
\text { Grid }\end{array}$} & \multirow[t]{2}{*}{$k$} & \multicolumn{2}{|c|}{$\begin{array}{l}\text { Woodbury vs. } \\
\text { Sparse-LU }\end{array}$} & \multicolumn{2}{|c|}{$\begin{array}{c}\text { Woodbury-Banachiewicz- } \\
\text { Schur vs. Sparse-LU }\end{array}$} \\
\hline Name & \# Nodes & & speed-up & $\kappa_{w}$ & speed-up & $\kappa_{w b s}$ \\
\hline IBMPG2 & 61,677 & 65 & $8.20 \mathrm{x}$ & $2.24 \times 10^{-14}$ & $8.52 \mathrm{x}$ & $2.24 \times 10^{-14}$ \\
\hline IBMPG3 & 410,011 & 69 & $12.30 \mathrm{x}$ & $9.09 \times 10^{-8}$ & $12.23 \mathrm{x}$ & $9.09 \times 10^{-8}$ \\
\hline IBMPG4 & 474,524 & 69 & $16.45 \mathrm{x}$ & $4.60 \times 10^{-14}$ & $16.87 x$ & $4.60 \times 10^{-14}$ \\
\hline IBMPG5 & 248,838 & 61 & $8.51 \mathrm{x}$ & $9.44 \times 10^{-13}$ & $8.65 \mathrm{x}$ & $9.44 \times 10^{-13}$ \\
\hline IBMPG6 & 403,915 & 63 & $8.55 \mathrm{x}$ & $2.27 \times 10^{-13}$ & $8.67 \mathrm{x}$ & $2.27 \times 10^{-13}$ \\
\hline IBMPGNEW1 & 315,951 & 69 & $10.67 \mathrm{x}$ & $4.21 \times 10^{-15}$ & $10.76 \mathrm{x}$ & $4.21 \times 10^{-15}$ \\
\hline IBMPGNEW2 & 717,754 & 69 & $15.54 \mathrm{x}$ & $1.44 \times 10^{-7}$ & $15.67 \mathrm{x}$ & $1.44 \times 10^{-7}$ \\
\hline G1 & 50,444 & 55 & $9.72 \mathrm{x}$ & $1.92 \times 10^{-17}$ & $10.07 x$ & $1.92 \times 10^{-17}$ \\
\hline G2 & 113,306 & 69 & $8.65 \mathrm{x}$ & $9.03 \times 10^{-18}$ & $8.80 \mathrm{x}$ & $9.03 \times 10^{-18}$ \\
\hline G3 & 200,828 & 69 & $9.10 \mathrm{x}$ & $3.51 \times 10^{-17}$ & $9.39 \mathrm{x}$ & $3.51 \times 10^{-17}$ \\
\hline G4 & 449,182 & 69 & $10.37 x$ & $1.52 \times 10^{-17}$ & $10.75 x$ & $1.52 \times 10^{-17}$ \\
\hline G5 & $1,006,625$ & 59 & $13.05 \mathrm{x}$ & $3.07 \times 10^{-17}$ & $13.20 \mathrm{x}$ & $3.07 \times 10^{-17}$ \\
\hline \multicolumn{3}{|c|}{ Average } & $10.93 x$ & $1.96 \times 10^{-8}$ & $11.13 x$ & $1.96 \times 10^{-8}$ \\
\hline
\end{tabular}

Table 3.1: Comparison between Woodbury Formula Solution Update and Sparse-LU using UMFPACK

A 2.6 GHz Linux machine with $24 \mathrm{~GB}$ of RAM was used to obtain the results. For each grid, a random failure sequence of $k$ interconnects was decided, and then the node voltage drops were updated based on the pre-determined failure sequence in separate runs using Sparse-LU, Woodbury (eq. (3.10)) and Woodbury-Banachiewicz-Schur form (eq. (3.14) and (3.19)). The results are as tabulated in Table 3.1, which reports the speed-up obtained by Woodbury and Woodbury-Banachiewicz-Schur form over Sparse-LU, and the difference in node voltage drops in terms of $\kappa_{w}$ and $\kappa_{w b s} . \kappa_{w}$ is defined as follows: let $v_{p, l u}$ and $v_{p, w}$ be the voltage drop vectors obtained using Sparse-LU and Woodbury formula after the $p^{t h}$ interconnect failure, then:

$$
\kappa_{w}=\frac{\max _{p \in\{1,2 \ldots k\}}\left[\operatorname{abs}\left(\left\|v_{p, w}\right\|-\left\|v_{p, l u}\right\|\right)\right]}{m}
$$

where $\|\cdot\|$ represents the sum-norm and $m$ is the number of nodes in the grid. Similarly, $\kappa_{w b s}$ is defined as:

$$
\kappa_{w b s}=\frac{\max _{p \in\{1,2 \ldots k\}}\left[\operatorname{abs}\left(\left\|v_{p, w b s}\right\|-\left\|v_{p, l u}\right\|\right)\right]}{m}
$$




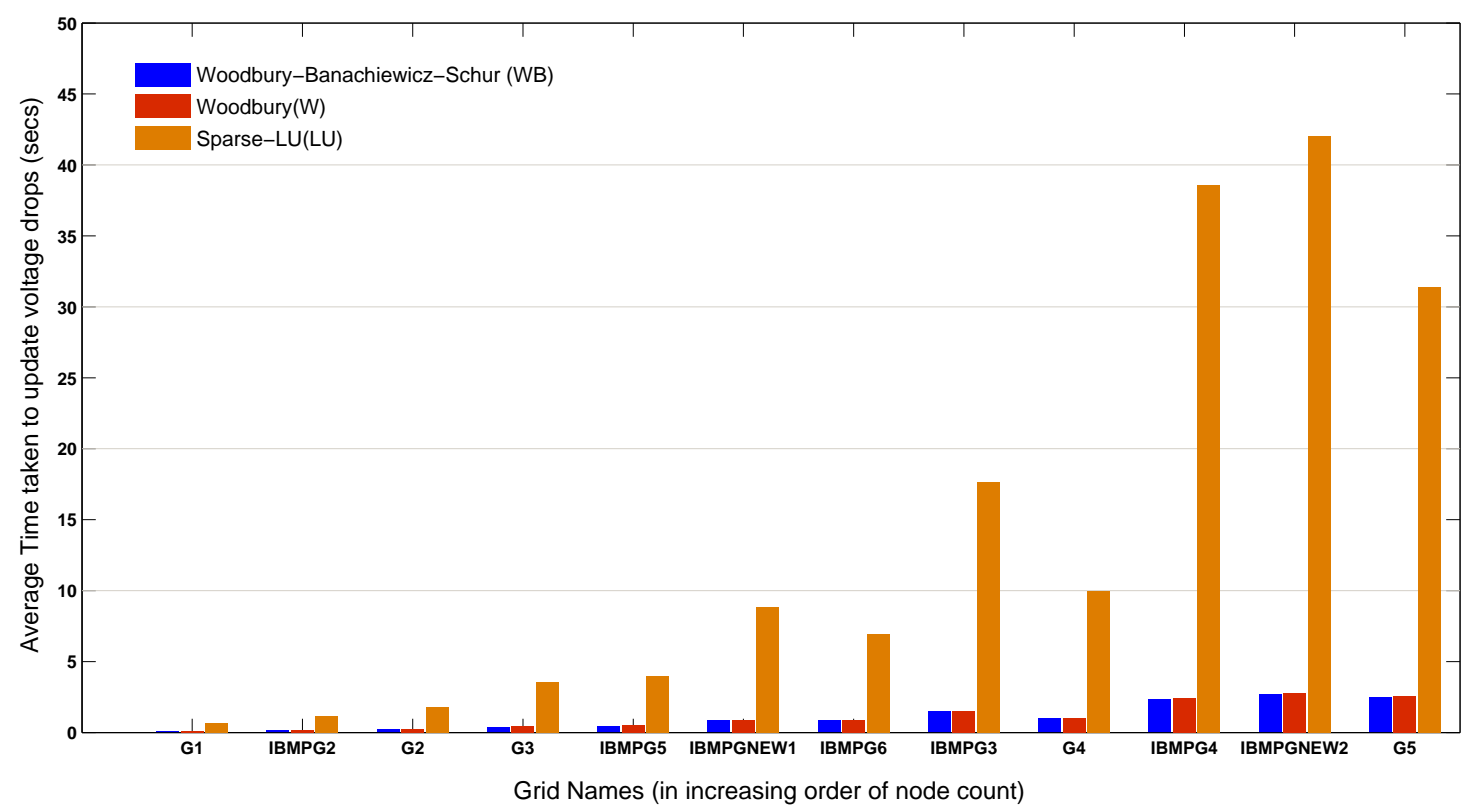

Figure 3.4: Comparison of average time taken to update the voltage drops

where $v_{p, w b s}$ is the voltage drop vector obtained using Woodbury-Banachiewicz-Schur formula after the $p^{t h}$ interconnect failure. For each grid, $\kappa_{w}\left(\kappa_{w b s}\right)$ gives an idea of the maximum difference in voltage drop calculation per node among all $k$ interconnect failures in case of Sparse-LU vs. Woodbury (Sparse-LU vs. Woodbury-Banchiewicz-Schur formula). From Table 3.1 , we clearly observe that that on an average, Woodbury formula obtains a significant speedup of 10.93x as compared to Sparse-LU with negligible error in calculation of voltage drop per node. Woodbury-Banachiewicz-Schur formula performs slightly better and has a speed up of $11.13 \mathrm{x}$, which is to be expected.

Figure 3.4 compares the average CPU-time taken by the three methods to find a new voltage drop vector. Figure 3.5 compares the cumulative time taken to update the node voltage drops up to $p^{\text {th }}$ interconnect failure for IBMPG4 grid. Lastly, figure 3.6 shows the empirically determined complexity for the three methods. Sparse-LU has the highest complexity of $\mathcal{O}\left(m^{1.41}\right)$, Woodbury has a reduced complexity of $\mathcal{O}\left(m^{1.22}\right)$ and Woodbury-Banachiewicz-Schur has a slightly improved complexity of $\mathcal{O}\left(m^{1.21}\right)$.

\subsection{A small note on the Mesh Model}

The mesh model tries to account for the redundancy in the power grid mesh structure, which provides many parallel paths for the current to flow from C4 bumps to the current sources. As mentioned before, the stress level of a grid is identified by its node voltage drop. To show that a power grid can handle more than one interconnect failure due to redundancy, we plot a voltage- 


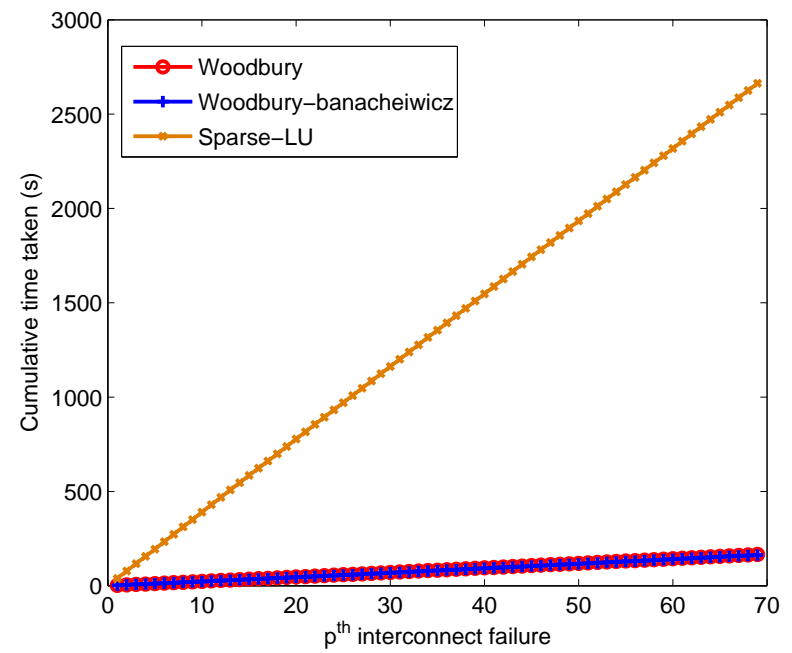

(a) Comparision of all three methods

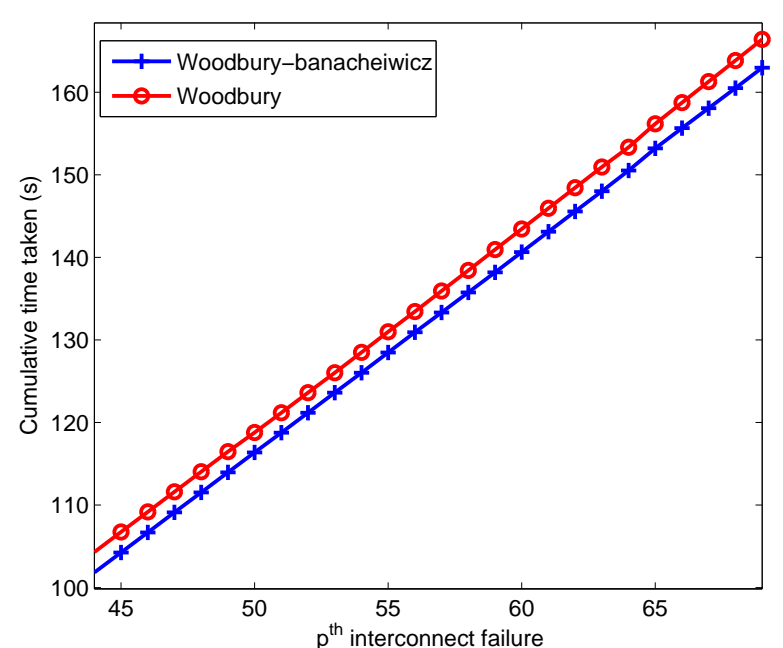

(b) Improvement of Woodbury-Banachiwicz-Schur form over Only Woodbury

Figure 3.5: Comparison of cumulative time taken to update the voltage drop after $p^{\text {th }}$ interconnect fails for grid IBMPG4
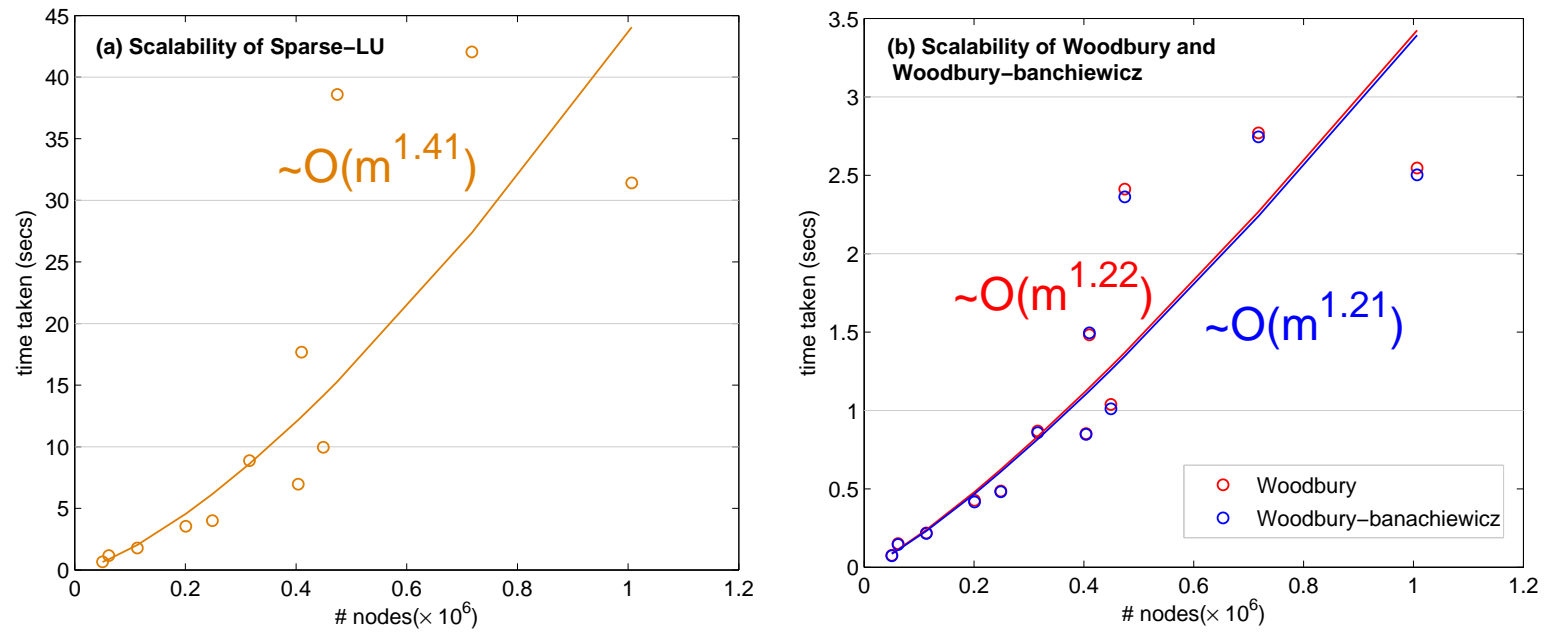

Figure 3.6: Empirically determined complexity of (a) Sparse-LU (UMFPACK) (b) Woodbury and Woodbury-Banachiewicz-Schur form 


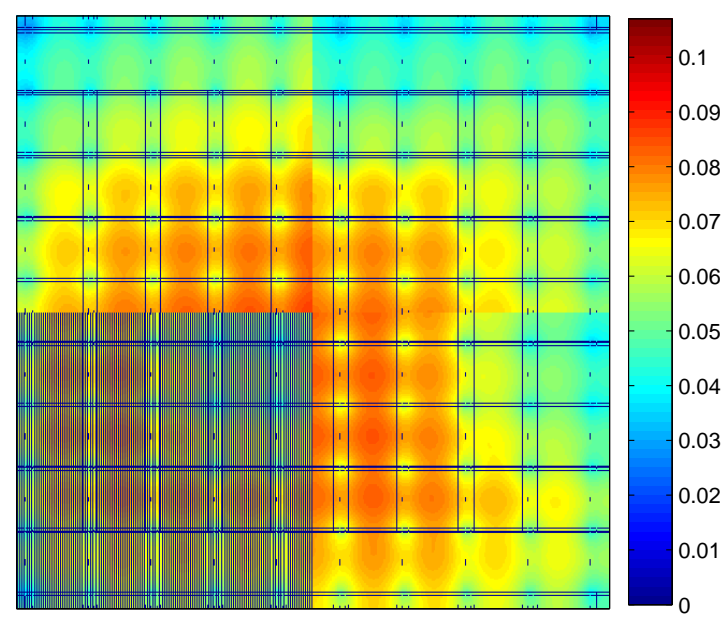

(a)

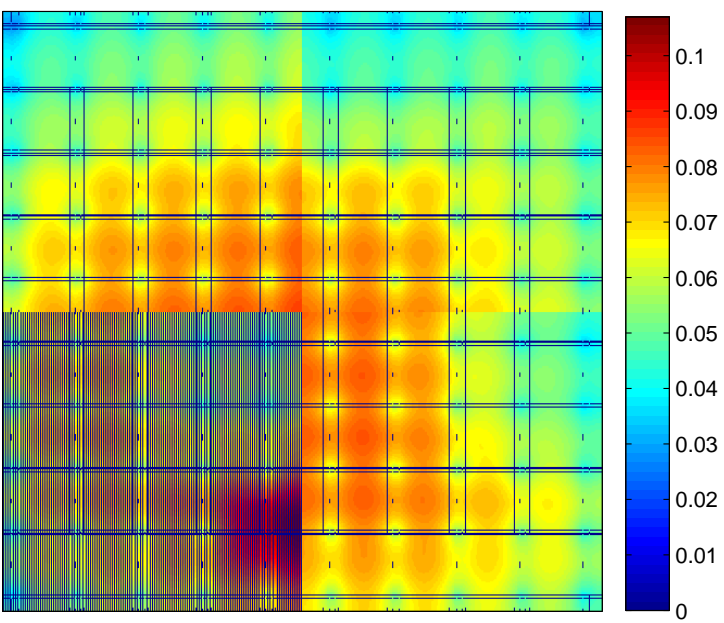

(b)

Figure 3.7: Voltage-drop maps of IBMPG5 grid (a) at $t=0$, when it is fresh and (b) after the first interconnect failure. As per series model, (b) has failed, but clearly, the voltage drops at all nodes are below $v_{t h}=0.18 \mathrm{~V}$

drop map of two test grids at $t=0$ (when they are fresh), and after the first interconnect failure. Figure 3.7 and 3.8 show the voltage-drop maps of IBMPG5 and IBMPG6, respectively. For both the grids, $v_{t h}=0.18 \mathrm{~V}$, which was not reached by failing a single interconnect. The voltagedrop maps clearly show that unless the fresh grid has high voltage drops (close to threshold $v_{t h}$ ), the impact of a single interconnect failure is not sufficient to cause timing violations of the underlying circuitry. More detailed results on the mesh model are presented in the next chapter. 


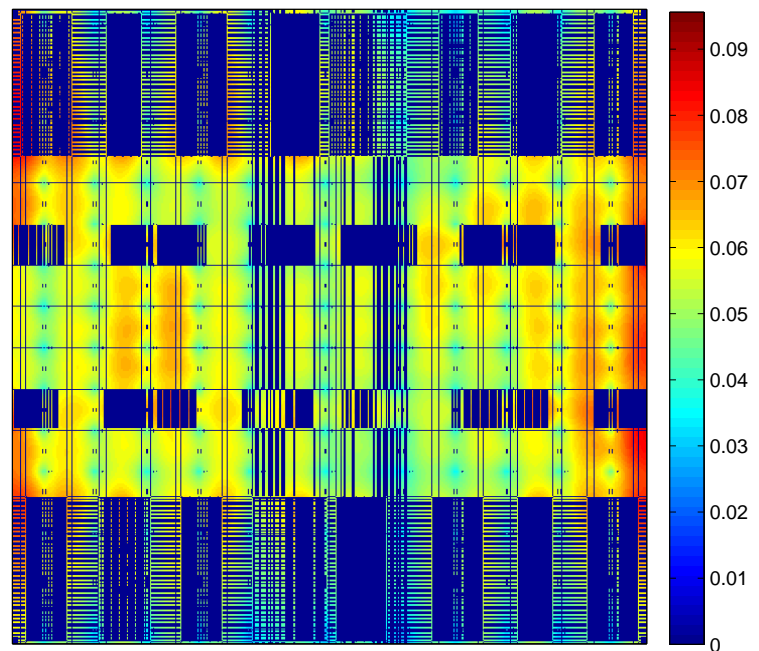

(a)

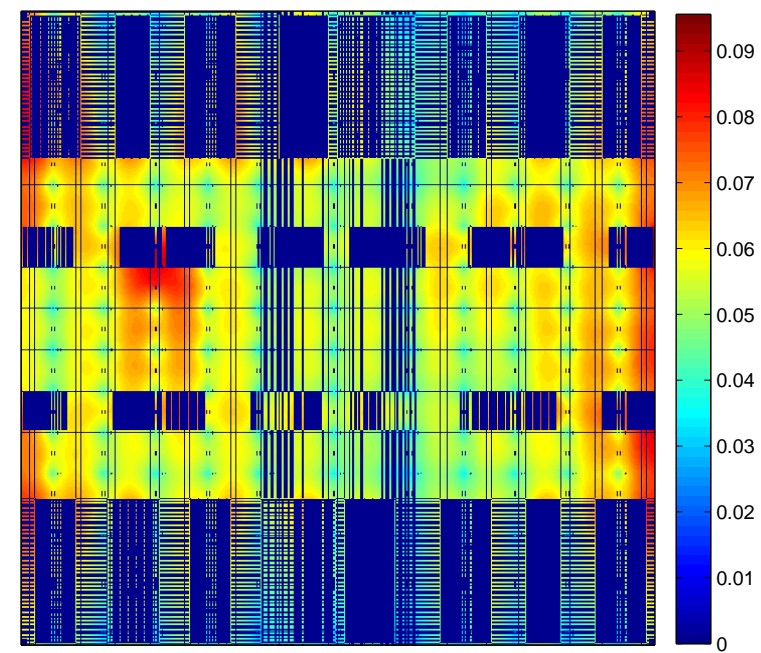

(b)

Figure 3.8: Voltage-drop maps of IBMPG6 grid (a) at $t=0$, when it is fresh and (b) after the first interconnect failure. As per series model, (b) has failed, but clearly, the voltage drops at all nodes are below $v_{t h}=0.18 \mathrm{~V}$ 


\section{Chapter 4}

\section{Estimating EM statistics for step currents}

\subsection{Introduction}

In the previous chapter, we introduced the mesh model, which accounted for the redundancies in the power grid while estimating its MTF. We also presented a highly efficient approach to update the node voltage drops after each interconnect failure. However, a key part was left out: an interconnect failure in the power grid changes the current-densities through all the surviving interconnects and hence affects their residual lifetime. Since the sparsity of the grid increases due to the failure of interconnects and the vector of source currents $i$ is constant, the surviving interconnects (on average) should conduct higher currents, which makes them more susceptible to failure due to EM. Thus, the failure statistics of the surviving interconnects should be modified to reflect the same. If ignored, it leads to an optimistic estimate of grid TTF, which is undesirable. In this chapter, we develop a novel approach to estimate the change in failure statistics of an interconnect when its effective-EM current density changes over time.

The chapter is organized as follows. Section 4.2 defines the problem and points out why the traditional effective-EM current model is inadequate to handle such a scenario. In section 4.3, the proposed approach is described in detail. This is followed by implementation in section 4.4 and results in section 4.5, where we compare the estimated grid MTF values as per the series and mesh model.

\subsection{Problem Definition}

Consider a thought experiment in which a large set $S_{0}$ of $N$ isolated conductors, made of the same material, using the same manufacturing process and with identical dimensions, are tested for their failure times. The testing starts at $t=0$. Let the current densities through all the 
conductors be identical and given by the following step function:

$$
J(t)= \begin{cases}J_{0}, & 0 \leq t \leq t_{1} \\ J_{1}, & t_{1}<t<\infty\end{cases}
$$

where $J_{0} \neq J_{1}$ and $t_{1}$ is large, such that many conductors from $S_{0}$ may have failed before $t_{1}$. This current profile is shown in Fig. 4.1a. One can also interpret this as a change in effective$E M$ current density over long time-periods. The population $S_{0}$ is fresh at $t=0$, but as time progresses, the conductors suffer damage due to EM and start failing. We are interested in determining the RV that describes the time-to-failure statistics of the population.

Unfortunately, the effective-EM current model (2.7) is not applicable in this case because a) It implicitly assumes that the resulting effective-EM current density is applied to all the conductors throughout their lifetime, and b) It assumes that the transient current-waveform is periodic.

The effective-EM current model is meant to handle periodic current waveforms that vary on a much smaller time-scale as compared to the lifetime of the conductors, such as transients occurring due to logic circuit operation. Whereas, in the proposed problem definition (4.1), the current is not periodic and it changes on a time-scale that is comparable to the TTFs of the conductors. Hence, many conductors might have failed exclusively due to $J_{0}$ before the current change happens. This motivates the need for a new approach to estimate the statistics of the surviving sub-population.

\subsection{Updating Interconnect statistics}

In this section, an approach to update the statistics of the TTF of a conductor as its current density steps on long time scales (comparable to its TTF) is presented. First, to motivate such an approach, the simple case of a single step change in current density as given in (4.1) is dealt with. Then, the underlying assumptions of such an approach are listed, and based on these assumptions, a more general framework to deal with the scenario of multiple step changes in current is developed. Finally, the concept of Blech length is incorporated into the proposed approach, and the relation to update the TTF of a conductor as its current-density changes is derived.

\subsubsection{The Single Step Case}

Consider another set $S_{1}$ of $N$ conductors identical to those in $S_{0}$. Suppose $S_{1}$ is subjected to a current density of $J_{1}$ for all $t \geq 0$, as shown in Fig. 4.1b. Let $F_{1}(t)$ be the cumulative distribution function $(\mathrm{CDF})$ of the population $S_{1}$ and $F_{0}(t)$ be the CDF of $S_{0}$. Clearly, $F_{0}(t)$ 


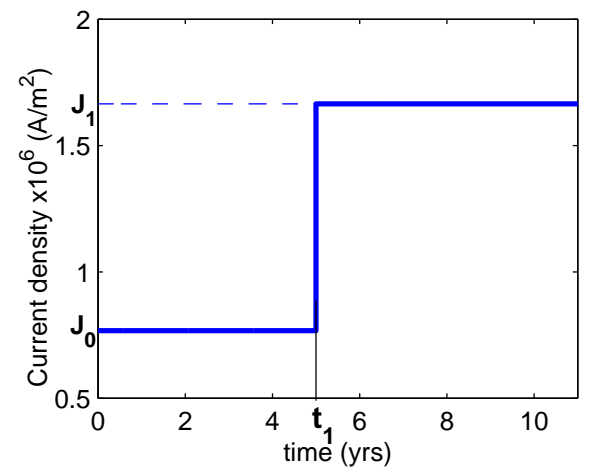

(a) Current density profile for $\mathrm{S}_{0}$

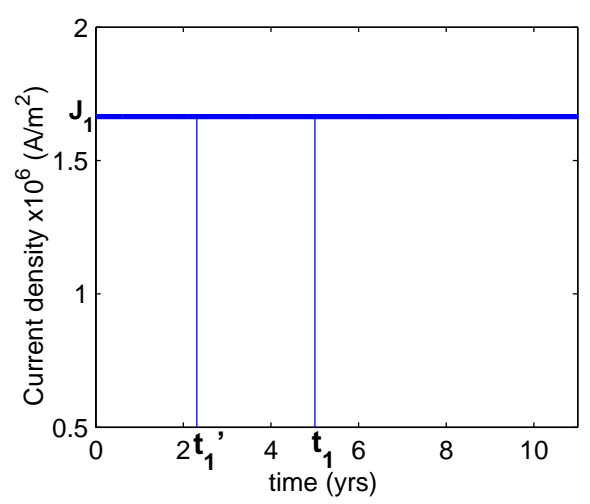

(b) Current density profile for $\mathrm{S}_{1}$

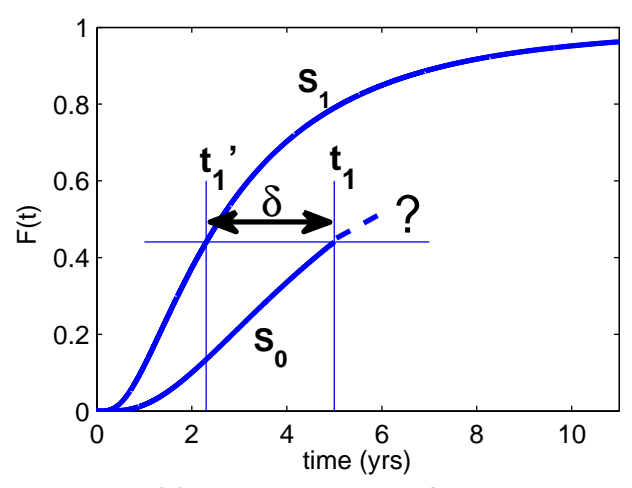

(c) what is the cdf for $S_{0}$ for $t>t_{1}$ ?

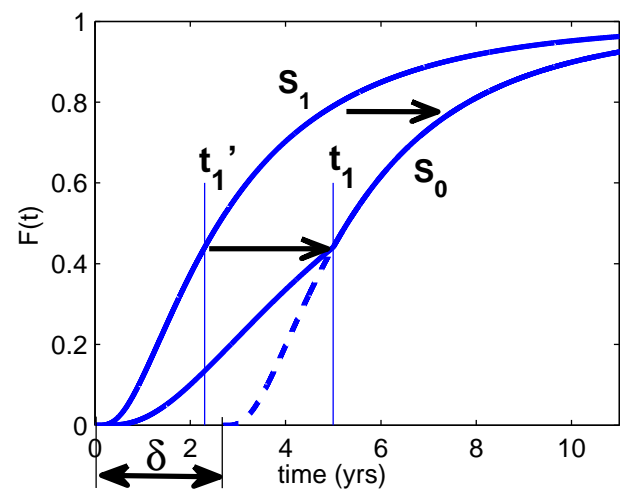

(d) The proposed solution

Figure 4.1: Proposed approach for single-step case, (a) Current density profile of $S_{0}$, (b) Current density profile of $S_{1}$, (c) Known CDF $F_{1}(t)$, CDF $F_{0}(t)$ unknown for $t>t_{1}$, (d) The proposed solution

for $t \leq t_{1}$ and $F_{1}(t) \forall t$ are known to be lognormal, and $F_{0}(t)$ for $t>t_{1}$ is to be determined. Define $t_{1}^{\prime}$ to be such that $F_{1}\left(t_{1}^{\prime}\right)=F_{0}\left(t_{1}\right)$ as shown in Fig. $4.1 \mathrm{c}$, where $t_{1}$ is the time of current change. The difference $\delta=t_{1}-t_{1}^{\prime}$ is easy to compute, as will be demonstrated later. For now, the key question is: 'what is the failure distribution of $S_{0}$ after $t_{1}$ ?' As already pointed out, traditional EM work is not helpful here. We now provide an argument to motivate a set of assumptions, which characterize the CDF of $S_{0}$ for $t>t_{1}$.

Proposition 2. The expected number of surviving members of a) $S_{0}$ at time $t_{1}$, and b) $S_{1}$ at time $t_{1}^{\prime}$, are exactly the same.

Proof. The two populations $S_{0}$ and $S_{1}$ started out fresh at $t=0$ with the same number of conductors $N$. Let $N_{s, 0}$ be the expected number of surviving conductors from the set $S_{0}$ for $t>t_{1}$ and $N_{s, 1}$ be the expected number of surviving conductors from the set $S_{1}$ for $t>t^{\prime}$. We know that $F_{1}\left(t^{\prime}\right)=F_{0}\left(t_{1}\right)$. Then, from definition (2.13), we have:

$$
\frac{N-N_{s, 1}}{N}=\frac{N-N_{s, 0}}{N} \Longrightarrow N_{s, 1}=N_{s, 0}
$$


Hence, the expected number of surviving members of $S_{0}$ and $S_{1}$ are exactly the same at $t_{1}$ and $t^{\prime}$, respectively.

Considering the two populations a) $S_{0}$ at time $t_{1}$, and b) $S_{1}$ at time $t_{1}^{\prime}$, we observe that:

1. The expected number of surviving members of the two populations are exactly the same, as proved in Proposition 2. Therefore, loosely speaking, the two populations have experienced an identical level of deterioration.

2. The two populations are subjected to exactly the same current stress $J_{1}$, as they move forward in time, i.e. $t_{1}+x$ and $t_{1}^{\prime}+x$, with $x \geq 0$ for $S_{0}$ and $S_{1}$, respectively.

Therefore, it is expected that, going forward in time, both populations will see the same instantaneous failure rate, i.e.:

$$
\begin{aligned}
& \lambda_{0}\left(t_{1}+x\right)=\lambda_{1}\left(t_{1}^{\prime}+x\right), \quad \forall x \geq 0 \\
& \text { or } \quad \lambda_{0}(t)=\lambda_{1}(t-\delta) \quad \text { for } t \geq t_{1}
\end{aligned}
$$

Since $\lambda_{1}(t)$ is the failure rate of a lognormal distribution, it follows that the failure rate of the surviving sub-population of $S_{0}$, i.e., $\lambda_{0}\left(t_{1}+x\right)$, is that of a lognormal. The use of a lognormal is justified because intrinsically, electromigration is a multiplicative degradation process (see section 2.3.2). Since this intrinsic property is unaffected by a change in current density, it is reasonable to assert that the TTF statistics of the surviving population are described by a lognormal.

Thus, it is proposed that the statistics of the surviving population of $S_{0}$ be obtained by shifting the origin of the lognormal that gives rise to $\lambda_{1}(t)$ by $\delta$ so that the continuity of $F_{0}(t)$ at $t=t_{1}$ is maintained, as shown in Fig. 4.1d. Hence, for $t>t_{1}$, the statistics of $S_{0}$ are described by a section of a shifted lognormal distribution, the mean of which is identical to the mean of the lognormal that gave rise to $\lambda_{1}(t)$. This key point motivates the first two assumptions to be made in the next section.

\subsubsection{Assumptions}

We now propose a set of assumptions motivated from the arguments in the previous section. These assumptions are then used to rigorously determine the CDF of $S_{0}$ for $t>t_{1}$ in the single-step case and the CDF in the general case of multiple change in currents.

Consider a population set $S$ that has already been subjected to some prior current density stress, i.e. it is not fresh. The following mild assumptions are made about $S$ for some time $t_{k} \neq 0$ :

Assumption 4.1. The statistics of the TTFs for the surviving population of $S$ are described by a (section of) shifted lognormal distribution. 
Assumption 4.2. The mean of the shifted lognormal distribution (relative to its start time) is given by Black's equation, with $J$ being the current density at time $t_{k}$.

Assumption 4.3. The value of $\sigma_{\ln }$ for $S$ at time $t_{k}$ is the same as that of the fresh population at $t=0$.

We note that assumption 4.3 can be dropped if the dependence of $\sigma_{\ln }$ with regard to the damage accumulated due to EM is known beforehand (see section 4.3.7).

\subsubsection{Determining $F_{0}(t)$ and $\delta$ for single-step case}

Based on the assumptions proposed in the previous section, we now rigorously obtain the CDF of $S_{0}$ for the single step case. Define two RVs $\mathbf{T}_{0}$ and $\mathbf{T}_{1}$ as follows:

1. $\mathbf{T}_{0}$ describes the TTF distribution of $S_{0}$ when it is subjected to $J_{0}$ for all $t \geq 0$.

2. $\mathbf{T}_{1}$ describes the TTF distribution when $S_{0}$ is subjected to zero current density for $t \leq \delta$ and $J_{1}$ for all $t>\delta$. In other words, the origin of the distribution is shifted to $t=\delta$.

Clearly, the CDFs $F_{T_{0}}(t)$ and $F_{T_{1}}(t)$ of $\mathbf{T}_{0}$ and $\mathbf{T}_{1}$ are known:

1. $\mathbf{T}_{0}$ has a lognormal distribution as given in (2.23).

2. $\mathbf{T}_{1}$ has a shifted-lognormal distribution as given in (2.24) with $\Delta=\delta$.

The MTF (relative to the origin of the distribution in each case) is given by Black's equation and the variance is experimentally determined. Using the proposed assumptions, the CDF of $S_{0}$ when subjected to a current-density profile of (4.1) is:

$$
F_{0}(t)= \begin{cases}F_{T_{0}}(t), & 0 \leq t \leq t_{1} \\ F_{T_{1}}(t-\delta), & t_{1}<t<\infty\end{cases}
$$

where the time-shift $\delta \in\left(-\infty, t_{1}\right)$ is necessary for maintaining the continuity constraint:

$$
\begin{aligned}
F_{T_{1}}\left(t_{1}-\delta\right) & =F_{T_{0}}\left(t_{1}\right) \\
\Phi\left[\frac{\ln \left(t_{1}-\delta\right)-\mu_{\ln , 1}}{\sigma_{\ln , 1} \sqrt{2}}\right] & =\Phi\left[\frac{\ln t_{1}-\mu_{\ln , 0}}{\sigma_{\ln , 0} \sqrt{2}}\right]
\end{aligned}
$$

where $\Phi$ the is standard normal CDF, $\mu_{\ln , \mathrm{k}}=E\left[\ln \mathbf{T}_{k}\right]$ and $\sigma_{\ln , \mathrm{k}}^{2}=\operatorname{Var}\left(\ln \mathbf{T}_{k}\right)$. The CDF $F_{0}(t)$ needs to be continuous because the TTF of a conductor can assume any value in a given time-range. 
Define $\mu_{\mathrm{T}, \mathrm{k}}=E\left[\mathbf{T}_{k}\right]$. Since $\Phi$ is monotonic, the equality will hold provided the terms in brackets are equal. Thus:

$$
\left(\frac{\ln \left(t_{1}-\delta\right)-\mu_{\ln , 1}}{\sigma_{\ln , 1} \sqrt{2}}\right)=\left(\frac{\ln t_{1}-\mu_{\ln , 0}}{\sigma_{\ln , 0} \sqrt{2}}\right)
$$

Use (2.26) in (4.5) to arrive at:

$$
\begin{aligned}
& \frac{\ln \left(t_{1}-\delta\right)-\left(\ln \mu_{\mathrm{T}, 1}-0.5 \sigma_{\ln , 1}^{2}\right)}{\sigma_{\ln , 1} \sqrt{2}}=\frac{\ln t_{1}-\left(\ln \mu_{\mathrm{T}, 0}-0.5 \sigma_{\ln , 0}^{2}\right)}{\sigma_{\ln , 0} \sqrt{2}} \\
& \frac{1}{\sigma_{\ln , 1}} \ln \left(\frac{t_{1}-\delta}{\mu_{\mathrm{T}, 1}}\right)+0.5 \sigma_{\ln , 1}=\frac{1}{\sigma_{\ln , 0}} \ln \left(\frac{t_{1}}{\mu_{\mathrm{T}, 0}}\right)+0.5 \sigma_{\ln , 0} \\
& \ln \left(\frac{t_{1}-\delta}{\mu_{\mathrm{T}, 1}}\right)=\frac{\sigma_{\ln , 1}}{\sigma_{\ln , 0}} \ln \left(\frac{t_{1}}{\mu_{\mathrm{T}, 0}}\right)+0.5 \sigma_{\ln , 1}\left(\sigma_{\ln , 0}-\sigma_{\ln , 1}\right)
\end{aligned}
$$

Let $\varphi=\frac{\sigma_{\ln , 1}}{\sigma_{\ln , 0}}$. Then, (4.6) becomes:

$$
\delta=t_{1}-\mu_{\mathrm{T}, 1}\left(\frac{t_{1}}{\mu_{\mathrm{T}, 0}}\right)^{\varphi} \exp \left(\frac{\sigma_{\ln , 0}^{2} \varphi(1-\varphi)}{2}\right)
$$

Using Assumption 4.3, $\sigma_{\ln , 0}=\sigma_{\ln , 1}$ or $\varphi=1$. This leads to:

$$
\delta=t_{1}\left[1-\frac{\mu_{\mathrm{T}, 1}}{\mu_{\mathrm{T}, 0}}\right]
$$

From Black's equation (2.1), it is known that $\frac{\mu_{\mathrm{T}, 1}}{\mu_{\mathrm{T}, 0}}=\left(\frac{J_{0}}{J_{1}}\right)^{n}$, the relative time-shift $\delta$ between $\mathbf{T}_{1}$ and $\mathbf{T}_{0}$ is:

$$
\delta=t_{1}\left[1-\left(\frac{J_{0}}{J_{1}}\right)^{n}\right]
$$

\subsubsection{The Case of Multiple changes in Currents}

Consider a second thought experiment with $S_{0}$ for which the current density profile is given as:

$$
J(t)=\left\{\begin{array}{cc}
J_{0}, & 0 \leq t \leq t_{1} \\
J_{1}, & t_{1}<t \leq t_{2} \\
\vdots & \\
J_{k-1}, & t_{k-1}<t \leq t_{k} \\
J_{k}, & t_{k}<t \leq t_{k+1} \\
\vdots &
\end{array}\right.
$$




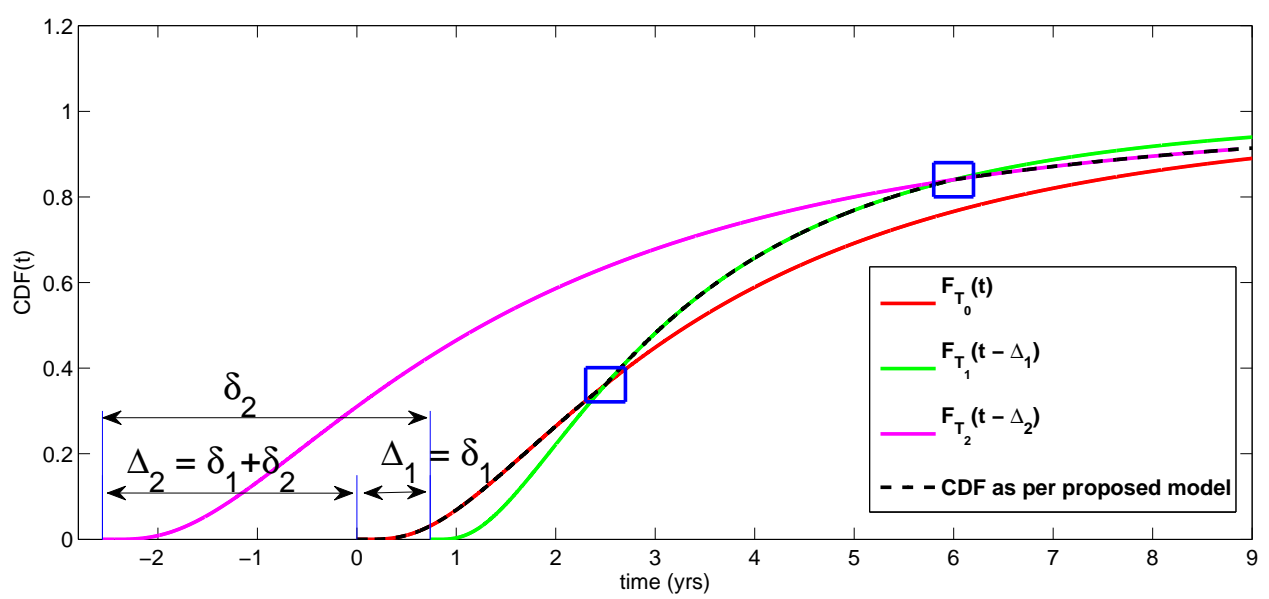

(a) CDF plot for $\mathrm{S}_{0}$ undergoing multi-step current density

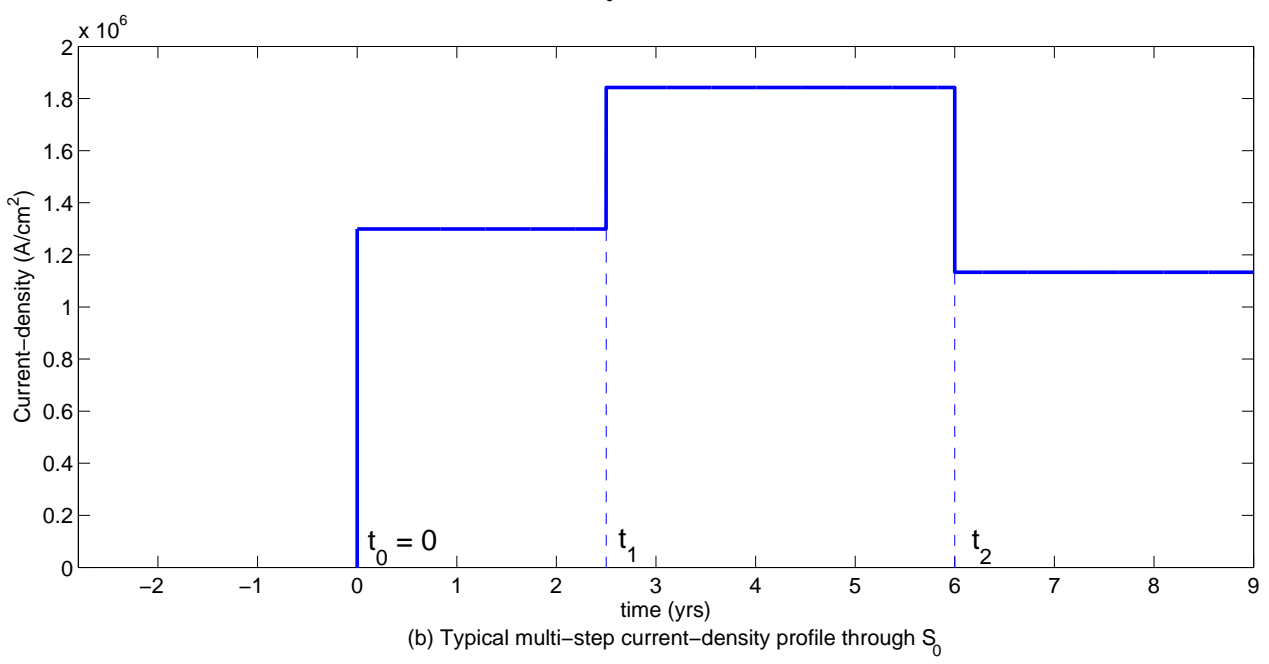

Figure 4.2: Proposed approach for multi-step case, (a) CDF plot of $S_{0}$ as per the proposed approach, the square markers locate the time of current change when a new shifted lognormal is used to describe the statistics, (b) Current density profile

where $J_{k-1} \neq J_{k} \forall k>0$. It is interesting to note that (4.10) is the typical current density profile of a surviving interconnect in the power grid, where the $k^{t h}$ failing interconnect has $\tau=t_{k}$. The analysis in this section assumes $J_{k} L>\beta_{c} \forall k$, i.e all the interconnects are EM-susceptible for all time-spans. The Blech-effect is incorporated in the next section.

The statistics of $S_{0}$ are derived based on the stated assumptions in the previous section. As per Assumption 4.1, for each time-span $t_{k}<t \leq t_{k+1}$, the statistics are described by a RV $\mathbf{T}_{k}$ that has a lognormal distribution originating at some $t=\Delta_{k}$. Assumption 4.2 dictates that the mean $\mu_{\mathrm{T}, \mathrm{k}}=E\left[\mathbf{T}_{k}\right]$ is given by Black's Eq. with $J=J_{k} . \Delta_{k}$ is determined as follows: in order to satisfy the continuity constraint, each RV $\mathbf{T}_{k}$ has a finite time-shift of $\delta_{k}$ with respect to $\mathbf{T}_{k-1}$, with $\mathbf{T}_{0}$ having a shift of $\delta_{0}=0$. This clearly implies that if the distribution for $\mathbf{T}_{k}$ 
originates at time $\Delta_{k}$ with respect to $t=0$, then $\Delta_{k}$ is:

$$
\Delta_{k}=\sum_{i=0}^{k} \delta_{i}
$$

The time-shift $\delta_{k}$ between $\mathbf{T}_{k}$ and $\mathbf{T}_{k-1}$ can be found using the continuity constraint:

$$
\Phi\left[\frac{\ln \left(t_{k}-\Delta_{k}\right)-\mu_{\ln , \mathrm{k}}}{\sigma_{\ln , \mathrm{k}} \sqrt{2}}\right]=\Phi\left[\frac{\ln \left(t_{k}-\Delta_{k-1}\right)-\mu_{\ln , \mathrm{k}-1}}{\sigma_{\ln , \mathrm{k}-1} \sqrt{2}}\right]
$$

Using $\Delta_{k}=\Delta_{k-1}+\delta_{k}$, assumption $4.3\left(\sigma_{\ln , 0}=\ldots=\sigma_{\ln , \mathrm{n}}=\sigma_{\ln }\right)$ and $(2.26)$ as before for the single step case, we have:

$$
\ln \left(\frac{t_{k}-\Delta_{k-1}-\delta_{k}}{\mu_{\mathrm{T}, \mathrm{k}}}\right)=\ln \left(\frac{t_{k}-\Delta_{k-1}}{\mu_{\mathrm{T}, \mathrm{k}-1}}\right)
$$

By equating the terms in brackets, this can be simplified to:

$$
\delta_{k}=\left(t_{k}-\Delta_{k-1}\right)\left(1-r_{k}\right)=\left(t_{k}-\sum_{i=1}^{k-1} \delta_{i}\right)\left(1-r_{k}\right)
$$

where $r_{k}=\left(\frac{\mu_{\mathrm{T}, \mathrm{k}}}{\mu_{\mathrm{T}, \mathrm{k}-1}}\right)=\left(\frac{J_{k-1}}{J_{k}}\right)^{n}$. The cdf of $S_{0}$ can now be written as:

$$
F_{0}(t)=\left\{\begin{array}{cc}
F_{T_{0}}\left(t-\Delta_{0}\right), & 0 \leq t \leq t_{1} \\
F_{T_{1}}\left(t-\Delta_{1}\right), & t_{1}<t \leq t_{2} \\
\vdots & \\
F_{T_{k-1}}\left(t-\Delta_{k-1}\right), & t_{k-1}<t \leq t_{k} \\
F_{T_{k}}\left(t-\Delta_{k}\right), & t_{k}<t \leq t_{k+1} \\
\vdots &
\end{array}\right.
$$

Figure 4.2 shows a typical CDF plot of $S_{0}$ for multi-step current density profile as per the proposed approach. From the figure, it can be easily observed that

- if $J_{k}>J_{k-1}$, the CDF slope increases to mirror the fact that the rate of degradation has increased after an increase in the current density, and

- if $J_{k}<J_{k-1}$, the CDF slope decreases to mirror the fact that the rate of degradation has decreased after a decrease in the current density.

Note that the proposed assumptions are robust, in the sense that if $J_{k-1}=J_{k}, \delta_{k}=0$ and the CDF of $S_{0}$ is given by a single lognormal distribution with MTF as obtained from Black's 


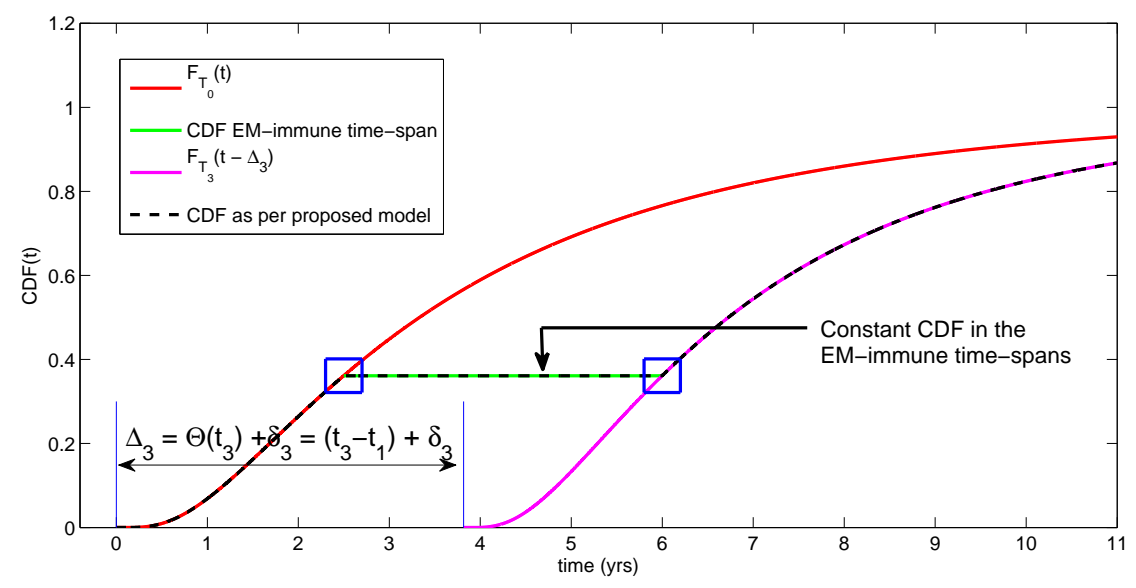

(a) CDF plot for a conductor undergoing current changes, with EM-immune time-spans in between

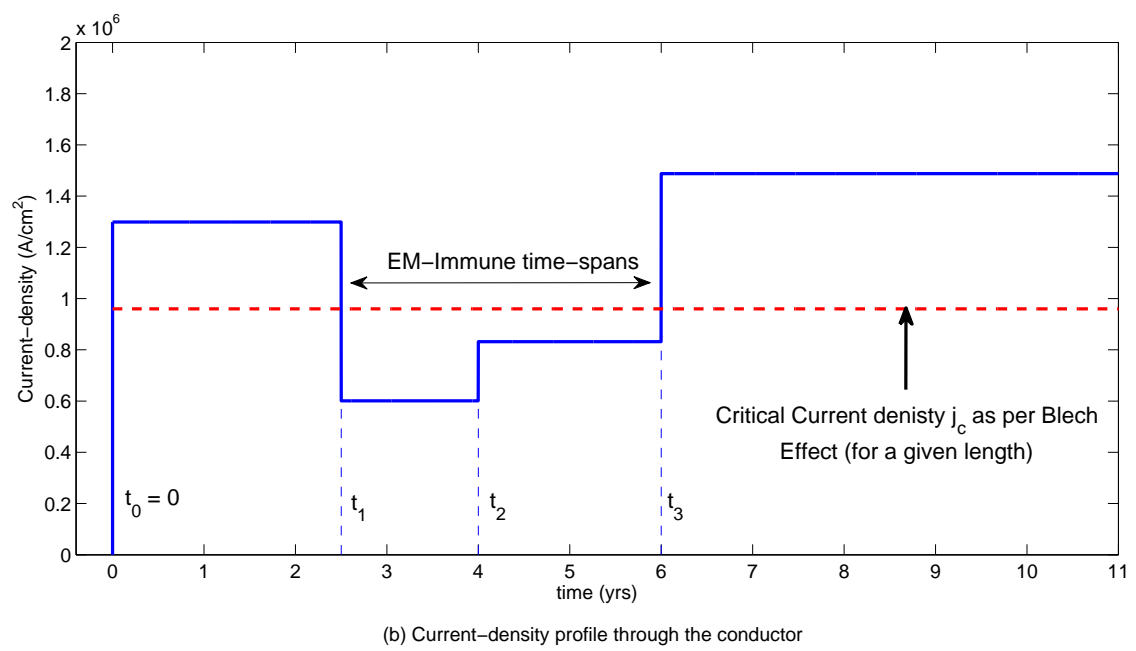

Figure 4.3: Proposed approach for multi-step case, with EM-immune time-spans in between (a) CDF plot of $S_{0}$ as per the proposed approach (b) Current density profile

equation.

The previous analysis assumed $J_{k} L>\beta_{c} \forall k$, where $\beta_{c}$ is given by (see (2.5)):

$$
\beta_{c}=\frac{\Omega}{Z^{*} e \rho}(\Delta \Lambda)
$$

$\Omega$ is the atomic volume, $\rho$ is the resistivity of the material, $Z^{*}$ is the effective atomic charge, $e$ is the fundamental atomic charge, $\Delta \Lambda$ is the stress difference within the interconnect. In the next section, we examine a more general scenario and incorporate the Blech effect into our analysis.

\subsubsection{Incorporating the Blech Effect}

Let $M$ and $B$ be the set of integers $k$, where $k$ denotes the time-span $t_{k}<t \leq t_{k+1}$, so that $M=\left\{k: J_{k} L \leq \beta_{c}\right\}$ is the set of EM-immune time-spans and $B=\left\{k: J_{k} l>\beta_{c}\right\}$ is the set of 
EM-susceptible time-spans. Clearly, $M \cap B=\emptyset$ and $M \cup B$ is the entire time period. In the previous section, it was assumed that $M=\emptyset$. Now consider a new thought experiment with $S_{0}$ where EM-immune and EM-susceptible time-spans interspersed with each other.

Consider a general scenario in which $(p-1)$ consecutive EM-immune time-spans are sandwiched between two EM-susceptible time-spans. To be precise, $\{k-p, k\} \in B$ and $\{k-p+$ $1, \ldots k-1\} \in M$. The surviving conductors (with $\tau>t_{k-p+1}$ ), will not fail in the time-spans $\{k-p+1, \ldots k-1\}$ because the current densities $J_{k-p+1}, \ldots J_{k-1}$ cannot generate sufficient stress for failure, regardless of state of degradation. Hence, the reliability/CDF of the population $S_{0}$ in the EM-immune time-spans does not change and remains the same as it was at the on-set of the corresponding EM-immune time-span. Based on this observation, the framework developed in the last section is extended to incorporate the Blech effect by introducing two modifications, as detailed below.

First, assumption 4.1 is now applicable only for EM-susceptible time-spans, so that RV $\mathbf{T}_{k}$, that has a shifted lognormal distribution originating at $t=\Delta_{k}$, exists for $k \in B$. Since the surviving conductors do not fail for $k \in M$, the corresponding probability of failure is zero and the associated CDF is a constant function. As $k \in B$ is encountered, the conductors start failing again. Thus, the CDF of $S_{0}$ in this case is defined to be:

$$
F_{0}(t)= \begin{cases}0 & k \in M: k<\min (B) \\ F_{T_{b}}\left(t_{b+1}-\Delta_{b}\right), & k \in M, b \in B: b<k \text { and }|k-b| \text { is minimum } \\ F_{T_{k}}\left(t-\Delta_{k}\right), & k \in B\end{cases}
$$

Second, the time of origin of the distribution for $\mathbf{T}_{k}$ is modified to take into account the EM-immune time-spans:

$$
\Delta_{k}=\Theta\left(t_{k}\right)+\sum_{i=0, i \in B}^{k} \delta_{i}
$$

where $\Theta(t)$ is the sum of all EM-immune time-spans upto the present time $t$, i.e.:

$$
\Theta(t)=\sum_{k \in Q}\left(t_{k+1}-t_{k}\right) \text { s.t. } Q=\left\{k: k \in M \text { and } t_{k+1}<t\right\}
$$

and $\delta_{k}$ is time-shift of $\mathbf{T}_{k}$ relative to $\mathbf{T}_{k-p}$ needed to maintain the continuity constraint if the in-between EM-immune time-spans $\{k-p+1, \ldots k-1\}$ are removed. $\delta_{k}$ is found using the continuity constraint, which in this case is:

$$
\Phi\left[\frac{\ln \left(t_{k}-\Delta_{k}\right)-\mu_{\ln , \mathrm{k}}}{\sigma_{\ln , \mathrm{k}} \sqrt{2}}\right]=\Phi\left[\frac{\ln \left(t_{k-p+1}-\Delta_{k-p}\right)-\mu_{\ln , \mathrm{k}-\mathrm{p}}}{\sigma_{\ln , \mathrm{k}-\mathrm{p}} \sqrt{2}}\right]
$$

Equating the terms in the brackets and using assumption $4.3\left(\sigma_{\ln , 0}=\ldots=\sigma_{\ln , \mathrm{n}}=\sigma_{\ln }\right)$ and 
(2.26) as before, we get:

$$
\ln \left(\frac{t_{k}-\Delta_{k}}{\mu_{\mathrm{T}, \mathrm{k}}}\right)=\ln \left(\frac{t_{k-p+1}-\Delta_{k-p}}{\mu_{\mathrm{T}, \mathrm{k}-\mathrm{p}}}\right)
$$

Since logarithm is also a monotonic function, we can equate the terms in brackets to get:

$$
t_{k}-\Delta_{k}=\left(t_{k-p+1}-\Delta_{k-p}\right) r_{k}
$$

where $r_{k}=\left(\frac{\mu_{\mathrm{T}, \mathrm{k}}}{\mu_{\mathrm{T}, \mathrm{k}-\mathrm{p}}}\right)=\left(\frac{J_{k-p}}{J_{k}}\right)^{n}$ in this case. From definition, it is known that:

$$
\Delta_{k-p}=\Theta\left(t_{k-p}\right)+\sum_{i=0, i \in B}^{k-p} \delta_{i}
$$

Subtracting (4.19) from (4.15), $\Delta_{k}$ can be expressed in terms of $\Delta_{k-p}$ :

$$
\begin{aligned}
\Delta_{k}-\Delta_{k-p} & =\Theta_{k}-\Theta_{k-p}+\sum_{i=0, i \in B}^{k} \delta_{i}-\sum_{i=0, i \in B}^{k-p} \delta_{i} \\
& =\left(t_{k}-t_{k-p+1}\right)+\delta_{k}
\end{aligned}
$$

or

$$
\Delta_{k}=\Delta_{k-p}+\left(t_{k}-t_{k-p+1}\right)+\delta_{k}
$$

Substituting the value of $\Delta_{k}$ in (4.18):

$$
\begin{aligned}
t_{k}-\left(\Delta_{k-p}+\left(t_{k}-t_{k-p+1}\right)+\delta_{k}\right) & =\left(t_{k-p+1}-\Delta_{k-p}\right) r_{k} \\
\left(t_{k-p+1}-\Delta_{k-p}\right)-\delta_{k} & =\left(t_{k-p+1}-\Delta_{k-p}\right) r_{k} \\
\delta_{k} & =\left(t_{k-p+1}-\Delta_{k-p}\right)\left(1-r_{k}\right)
\end{aligned}
$$

Clearly, (4.21) reduces to (4.11) for $p=1$. Figure 4.3 depicts the proposed model.

\subsubsection{Updating A TTF sample}

Consider a conductor $\mathcal{C}$ of the set $S_{0}$ subjected to the current density profile (4.10). Clearly, the TTF of $\mathcal{C}$ changes because the RV describing the statistics of the population changes for each time-span $t_{k}<t \leq t_{k+1}$. At $t_{0}(=0), \mathcal{C}$ has a TTF given by (using (2.30)):

$$
\tau_{0}=\mu_{\mathrm{T}, 0} \cdot \exp \left(\Psi \sigma_{\ln }-0.5 \sigma_{\ln }^{2}\right)
$$

where $\Psi$ is a sample value from Standard Normal Distribution $\Phi, \mu_{\mathrm{T}, 0}=E\left[\mathbf{T}_{0}\right]$ and $\sigma_{\ln }^{2}=$ $\operatorname{Var}\left(\mathbf{T}_{0}\right) . \sigma_{\ln }$ is constant throughout the life of conductor. 


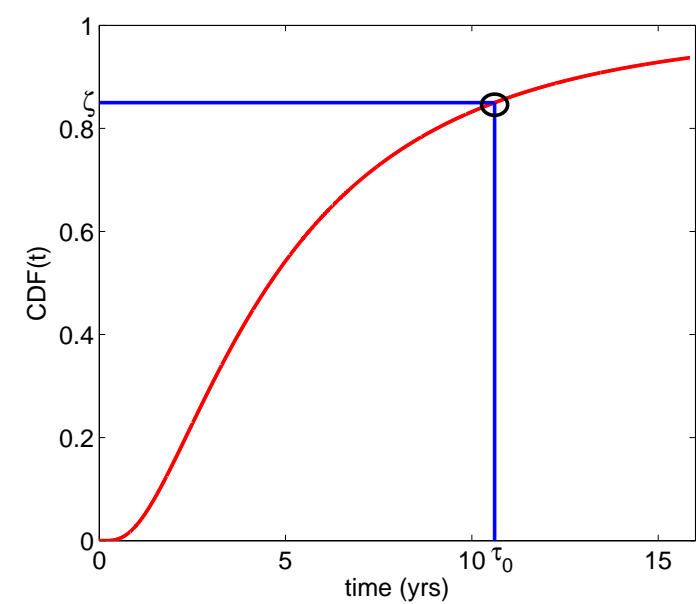

(a) Obtaining a TTF sample at $\mathrm{t}=0$

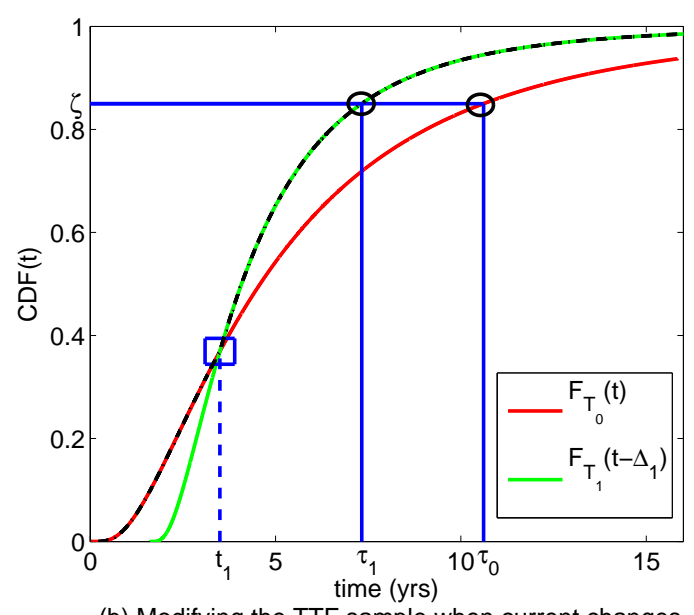

(b) Modifying the TTF sample when current changes

Figure 4.4: Updating a TTF sample (a) Obtaining a TTF sample at $t=0$ (b) Updating TTF sample when current density changes, the square marker locates the time of currentdensity change

To probe further, (4.22) can be re-written as:

$$
\tau_{0}=\mu_{\mathrm{T}, 0} \cdot \exp \left(\Phi^{-1}(\zeta) \sigma_{\ln }-0.5 \sigma_{\ln }^{2}\right)
$$

where $\zeta$ is a scalar between 0 and 1. From (4.23), one can view the process of assigning a TTF sample from a lognormal distribution as the inverse mapping of a uniformly selected random number from 0 to 1 . Graphically, this is shown in figure $4.4 \mathrm{a}$, where a selected point on the $\mathrm{y}$-axis $\left(\zeta\right.$, a CDF value) is inversely mapped from $F_{T_{0}}(t)$ to it's corresponding value, $\tau_{0}$, on the $\mathrm{x}$-axis (time axis).

When the current density changes through $\mathcal{C}$, the $\mathrm{CDF}$ is changed accordingly, as detailed in the previous text. The new TTF, $\tau_{1}$, is found in a similar way as before: $\zeta$ is inversely mapped from $F_{T_{1}}\left(t-\Delta_{1}\right)$ to it's corresponding value, $\tau_{1}$, on the x-axis. This is shown in figure $4.4 \mathrm{~b}$. The analytical expression for the same is:

$$
\tau_{1}=\Delta_{1}+\mu_{\mathrm{T}, 1} \exp \left(\Phi^{-1}(\zeta) \sigma_{\ln }-0.5 \sigma_{\ln }^{2}\right)
$$

Equation (4.24) can be generalized. Assume, for the sake of argument, that $\mathcal{C}$ survives for $t>t_{k}$ and $k \in B$. At $t=t_{k}$, when the $k^{t h}$ current change occurs, the TTF of $\mathcal{C}$ is updated using the following relation:

$$
\tau_{k}=\Delta_{k}+\mu_{\mathrm{T}, \mathrm{k}} \exp \left(\Phi^{-1}(\zeta) \sigma_{\mathrm{ln}}-0.5 \sigma_{\mathrm{ln}}^{2}\right)
$$

where $\zeta$ is the same as initially used in (4.23). The offset $\Delta_{k}$ (obtained from (4.20) or (4.15)) is added so that $\tau_{k}$ is now referred from $t=0$. For $k \in M, \tau_{k}$ is defined to be $\infty$. 
Finally, to verify the integrity of the proposed approach, it is proved that if the given framework is implemented, the new TTF will always be greater than the present time.

Theorem 3. Consider a conductor having the current density profile of (4.10). Let $\{k-p, k\} \in$ $B$ and $\{k-p+1, \ldots k-1\} \in M$. Then, if eq. (4.21) and (4.25) are used to find the offset $\left(\delta_{k}\right)$ and TTF $\left(\tau_{k}\right)$ for the conductor, we always have:

$$
\tau_{k}=t_{k}+\left(\tau_{k-p}-t_{k-p+1}\right)\left(\frac{J_{k-p}}{J_{k}}\right)^{n}
$$

so that $\tau_{k}>t_{k}$.

Proof. Let $\Upsilon=\exp \left(\Psi \sigma_{\ln }-0.5 \sigma_{\ln }^{2}\right)$. By Assumption 4.3, $\Upsilon$ will be the same throughout the life-time of the conductor.

As stated, the conductor was EM-susceptible at $t=t_{k-p}$, became EM-immune for all time $\operatorname{span}(\mathrm{s})$ following $t=t_{k-p+1}$ until $t=t_{k}$, when it becomes EM-susceptible again. Clearly, $\tau_{k-p}>t_{k-p+1}$, or the conductor should have failed before $t_{k-p+1}$. From theory, the RVs $\mathbf{T}_{k-p+1}, \ldots \mathbf{T}_{k-1}$ are undefined. From (4.15), the time of origin of $\mathbf{T}_{k}$ can be written as:

$$
\begin{aligned}
\Delta_{k} & =\Delta_{k-p}+\Theta\left(t_{k}\right)-\Theta\left(t_{k-p}\right)+\delta_{k} \\
& =\Delta_{k-p}+\left(t_{k}-t_{k-p+1}\right)+\left(t_{k-p+1}-\Delta_{k-p}\right)\left(1-r_{k}\right) \\
& =t_{k}-r_{k} t_{k-p+1}+r_{k} \Delta_{k-p}
\end{aligned}
$$

where $r_{k}=\left(\frac{J_{k-p}}{J_{k}}\right)^{n}=\left(\frac{\mu_{\mathrm{T}, \mathrm{k}}}{\mu_{\mathrm{T}, \mathrm{k}-\mathrm{p}}}\right)$. The new TTF at $t=t_{k}$ can now be written as:

$$
\begin{aligned}
\tau_{k} & =\Delta_{k}+\mu_{\mathrm{T}, \mathrm{k}} \Upsilon \\
& =t_{k}-r_{k} t_{k-p+1}+r_{k} \Delta_{k-p}+r_{k} \mu_{\mathrm{T}, \mathrm{k}-\mathrm{p}} \Upsilon \\
& =t_{k}+\left(\Delta_{k-p}+\mu_{\mathrm{T}, \mathrm{k}-\mathrm{p}} \Upsilon-t_{k-p+1}\right) r_{k} \\
& =t_{k}+\left(\tau_{k-p}-t_{k-p+1}\right) r_{k}
\end{aligned}
$$

The result, that we update the residual life of a conductor after the current density changes, is a direct outcome of the assumptions 4.1 and 4.2. It makes sense because a change in current density at some time $t_{k} \neq 0$ can only affect the residual life of a conductor, the past statistics upto $t=t_{k}$ are unchanged.

\subsubsection{Dropping Assumption 4.3}

A small note on the applicability of the above framework if $\sigma_{\ln }$ is not considered constant: suppose the dependence of $\sigma_{\ln }$ with regard to the damage accumulated due to EM is known 
beforehand, so that one can determine $\sigma_{\ln }$ based on some measure of the state of degradation of the surviving conductors at the time of the current-density change. The new shifted lognormal distribution (used to describe the statistics of the surviving population after the current-density change) will thus have the new standard deviation. In such a case, we can argue that since CDF of a lognormal is a monotonically increasing function in time, the updated TTF determined using the first two assumptions and the continuity constraint will always be greater than the present time. For example, in figure $4.4 \mathrm{~b}, \tau_{1}>t_{1}$ even if the CDF $F_{T_{1}}\left(t-\Delta_{1}\right)$ has a different standard deviation.

\subsection{Implementation}

\subsubsection{Obtaining a grid TTF sample}

The overall flow for obtaining a sample of power grid TTF using the series and mesh model is given in Algorithm 2. The inputs to the algorithm are the factorized upper and lower triangular matrices of $G_{0}\left(L_{G}, U_{G}\right)$, the list of all interconnects in the grid $\left(\mathrm{R}_{\text {list }}\right)$, the vector of initial voltage drops at $t=0\left(v_{0}\right)$, the voltage drop threshold vector for all nodes of the grid $\left(v_{t h}\right)$, the critical Blech product $\left(\beta_{c}\right)$ and a flag enable_ttf_update which controls if the TTF updates are to be performed while obtaining the grid TTF sample. The outputs of the algorithm are $\tau_{s}$ and $\tau_{m}$, the grid TTF sample considering it as a series and mesh system, respectively.

Using a random number generator, sample TTFs are assigned to all the interconnects in the power grid based on their current densities at $t=0$. Considering the grid as a series system, failure of the first resistor should cause the grid to fail. Hence $\tau_{s}$ is assigned the TTF sample of the first resistor in the sorted list $\mathrm{R}_{\text {list }}$. In order to find the TTF sample of the grid using the mesh model $\left(\tau_{m}\right)$, we start failing resistors. The voltage drops are efficiently updated using the Woodbury-Banachiewicz formulation (Algorithm 1) and the TTFs of surviving interconnects are updated as outlined in section 4.3.5. The updated TTFs may change the sequence of failures, hence the surviving interconnects are re-ordered as per their updated TTFs to determine the next interconnect to fail. The interconnects keep failing until either node voltage drop(s) exceeds $v_{t h}$ or the grid becomes singular, after which the grid is deemed to have failed. $\tau_{m}$ is assigned the TTF sample of the last resistor, which caused the grid to fail.

\subsubsection{Estimating grid MTF}

The MTF of a grid, for both the series and the mesh model, is estimated by random sampling. In other words, Algorithm 2 is run $w$ times to generate $w$ grid TTF samples. The arithmetic mean of these samples is the estimate of the true mean $\mu$. The number of samples $w$ is decided based on the stopping criteria derived in section 2.5.1. The parameter values chosen in this case are $\alpha=0.05$ and $\epsilon=0.05$, i.e. there is a $95 \%$ confidence that the estimated MTF is within 


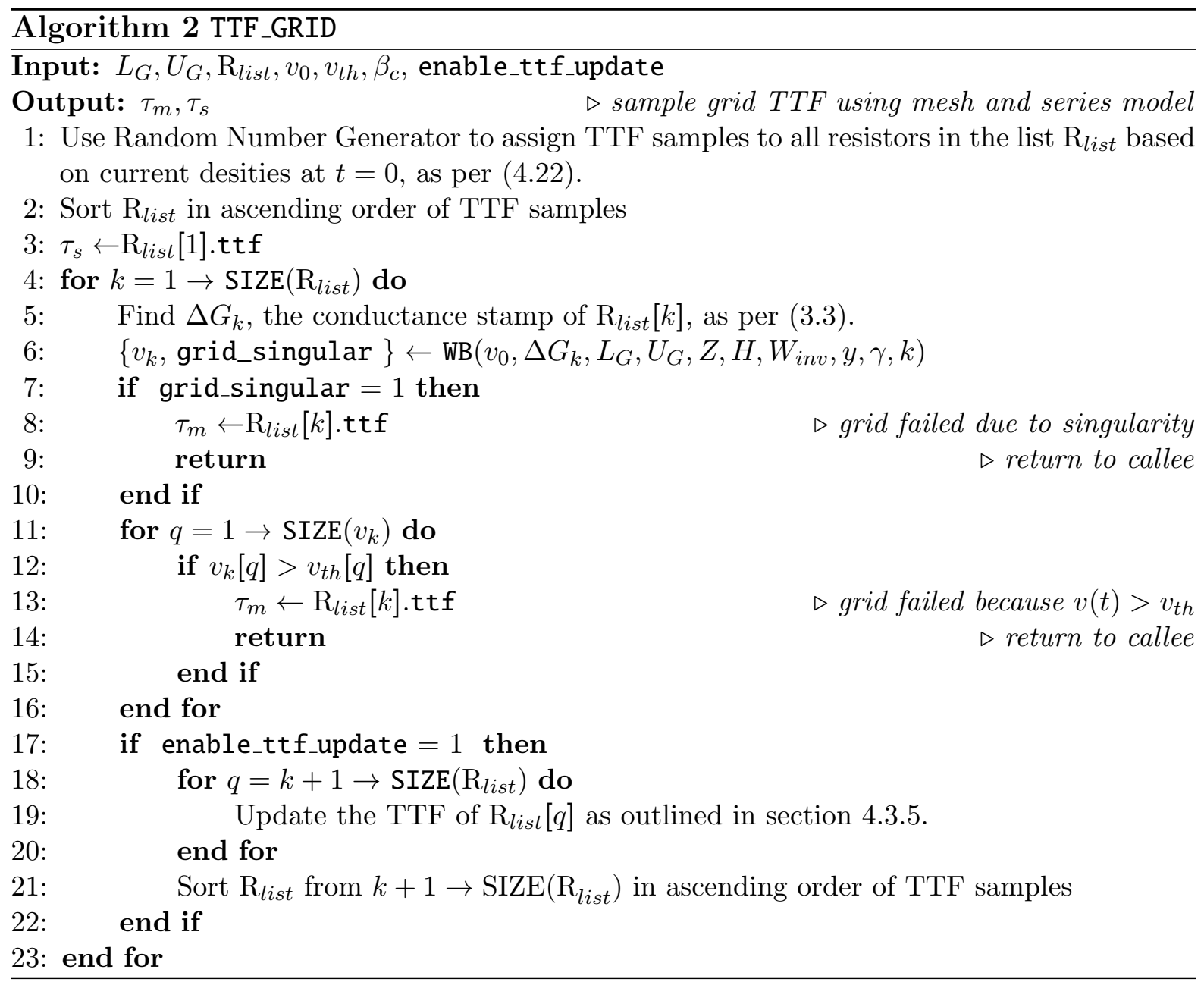

$5 \%$ of the true MTF for the respective model. Algorithm 3, MTF_GRID, computes the MTF as per the series and mesh models.

\subsection{Experimental Results}

A C++ implementation was written based on the proposed approach to estimate grid MTF as per the series and mesh models. As input, two types of test grids were used. The first type were generated per user specifications, including grid dimensions, metal layers, pitch and width per layer. The supply voltages and current sources were randomly placed on the grid. The technology specifications were consistent with $1.1 \mathrm{~V}$ 65nm CMOS technology. These grids are henceforth referred to as internal grids. The second type of grids are part of IBM power grid benchmarks [52]. These grids are dual grids (i.e. they have both $v_{d d}$ and gnd rails of the power grid), but the proposed approach was tested only for $v_{d d}$ part of the grids and are referred to as external grids. The grid details are given in Table 4.1.

The Voltage drop threshold was defined to be $10 \%$ of $v_{d d}$ for all nodes in a grid [44]. For 


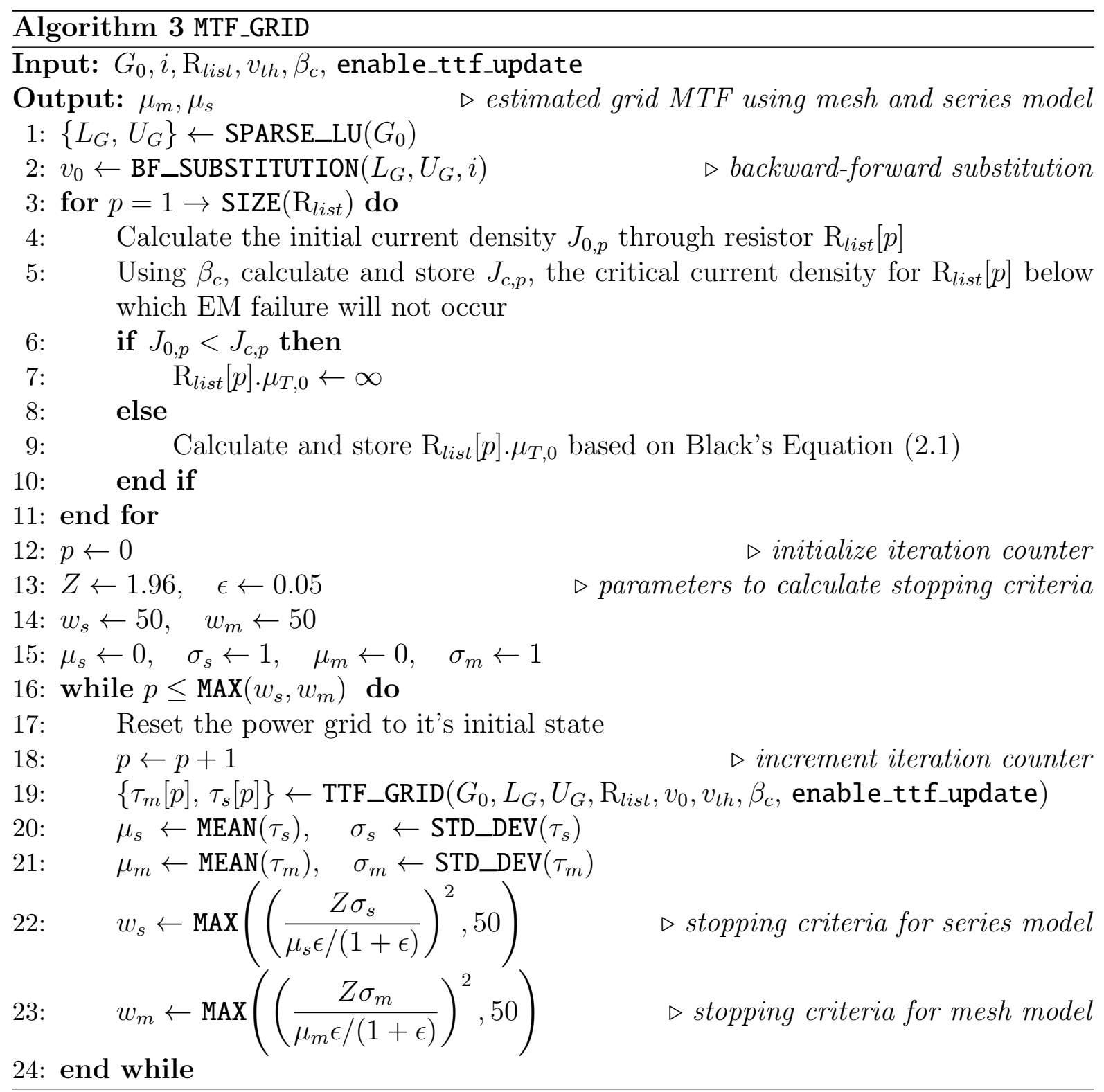

concreteness in results, the following configuration was assumed: the interconnect material is Aluminum (Al), with activation energy $E_{a}=0.9 \mathrm{eV}, \beta_{c}=3000 \mathrm{~A} / \mathrm{cm}$ [53] and a current exponent $n=1$, assuming a growth dominated failure due to the presence of shunt layers [54]. The standard deviation of the lognormal $\sigma_{\text {ln }}$ was assumed to be 0.81 for all interconnects in the grid, consistent with typical data in the literature [30] [31]. Since we use an empirical model to estimate the interconnect MTF, temperature enters into the analysis through Black's equation. In this work, a nominal interconnect temperature of $373 K$ was used in all simulations. Note that the proposed implementation can incorporate temperature/thermal profiles (obtained by using [55] or some other approach) as well. After each interconnect failure, the current density changes and hence the thermal profile needs to be updated. 
Table 4.1: Details of Power Grids used in experiments

\begin{tabular}{|c|c|c|c|c|c|}
\hline \multirow{2}{*}{ Grid Name } & \multicolumn{2}{|c|}{$\# v_{d d}$ Nodes } & \#voltage & \#current & \#inter- \\
& Before* & After** & sources & sources & connects \\
\hline IBMPG2 & 61,677 & 61,677 & 120 & 18,419 & 101,718 \\
\hline IBMPG3 & 410,011 & 410,011 & 494 & 100,527 & 677,388 \\
\hline IBMPG4 & 474,524 & 474,524 & 312 & 132,972 & 780,699 \\
\hline IBMPG5 & 497,558 & 248,838 & 100 & 236,600 & 495,756 \\
\hline IBMPG6 & 807,963 & 403,915 & 132 & 380,742 & 797,711 \\
\hline IBMPGNEW1 & 717,754 & 315,951 & 494 & 178,965 & 698,595 \\
\hline IBMPGNEW2 & 717,754 & 717,754 & 494 & 178,965 & $1,157,849$ \\
\hline G1 & 50,444 & 50,444 & 2,040 & 3,192 & 75,505 \\
\hline G2 & 113,306 & 113,306 & 4,400 & 7,140 & 169,160 \\
\hline G3 & 200,828 & 200,828 & 7,906 & 12,656 & 300,394 \\
\hline G4 & 449,182 & 449,182 & 17,400 & 28,056 & 671,599 \\
\hline G5 & $1,006,625$ & $1,006,625$ & 39,000 & 63,001 & $1,506,394$ \\
\hline
\end{tabular}

* Before means the original Grid Size

** After means the grid size after merging the short paths as equivalent node

A $2.6 \mathrm{GHz}$ Linux machine with $24 \mathrm{~GB}$ of RAM was used for all simulations. The experiments were carried out with two objectives: a) To establish that the mesh model reduces the pessimism in MTF estimation of the power grid, and b) To bring out the importance of the TTF update (TTFU) framework proposed in this chapter. To accomplish the same, algorithm MTF_GRID was run twice on each test grid, first with enable_ttf_updates $=1$ and second time with enable_ttf_updates $=0$. Table 4.2 tabulates the results of the first run, in which the statistics of all surviving interconnects are updated after each failure. This is the original approach, and the results from this run serve as a de-facto standard of comparison for other derivative approaches. Table 4.3 shows the results of the second run, in which the statistics are not updated for any interconnect failure. This is the secondary approach. Henceforth, $\mu_{m}^{*}$ denotes the grid MTF estimated from the original approach, and $\mu_{m}^{\prime}$ denotes the grid MTF estimated from the secondary approach.

The total time taken by either approach can be empirically expressed as:

$$
t_{c p u}=t_{i n i t}+\sum_{p=1}^{w_{m}} t_{m c, p}+t_{\text {extra }}
$$

where $t_{c p u}$ is the total time, $t_{\text {init }}$ is the initial one-time processing done to obtain the LU factorization and store the initial branch MTFs, $t_{m c, p}$ is the time taken to complete the $p^{\text {th }}$ Monte-carlo iteration and $t_{\text {extra }}$ is the extra time spent in obtaining grid TTF samples as per 
the series model if $w_{s}>w_{m}$. In practice, $t_{\text {init }}$ and $t_{\text {extra }}$ are very small as compared to the second term, hence

$$
t_{c p u} \approx \sum_{p=1}^{w_{m}} t_{m c, p}=w_{m} \times \overline{t_{m c}}=w_{m} \times \overline{t_{f}} \times \overline{k_{f}}
$$

where $\overline{t_{m c}}$ is the average time per Monte-carlo iteration, $\overline{t_{f}}$ is the average time spent in updating voltage drops and TTFs after an interconnect fails and $\overline{k_{f}}$ is the average number of interconnect failures required to fail the power grid.

Comparison between series and mesh model The power grid MTF as estimated using the series model and mesh model is compared using a gain ratio $\left(\mu_{m}^{*} / \mu_{s}\right)$. Among other things, the gain ratio is dependent on $\max \left(v_{0}\right)$, the maximum node voltage drop in the grid at $t=0$. If the difference between $\max \left(v_{0}\right)$ and $v_{t h}$ increases, the gain ratio also increases. If the difference is small, then the mesh model degenerates to series model. Given a reasonable difference between $v_{t h}$ and $\max \left(v_{0}\right)$, the gain ratio is found to be 3-4 for all the test grids, the average gain ratio being 3.78 for the test grids. Figure 4.5 graphically compares $\mu_{s}$ and $\mu_{m}^{*}$ for the test grids using a horizontal bar graph. It is observed that on average, large grids can tolerate upto 115 interconnect failures before the grid truly fails. These results clearly indicate that the series model, that declares a grid to be failed just after the first interconnect failure, is highly pessimistic for EM reliability verification.

Significance of TTF update framework Next, for the secondary run, the TTF updates are turned off. The accuracy of results and performance of the estimation engine is compared to the original approach, and is reported in table 4.3. The relative speed-up is expressed as a ratio of time taken in the original approach to the time taken in the secondary approach. The error in estimation is measured as

$$
\varepsilon^{\prime} \triangleq \frac{\left(\mu_{m}^{\prime}-\mu_{m}^{*}\right) \times 100}{\mu_{m}^{*}} \%
$$

The estimated MTF $\mu_{m}^{\prime}$ in the secondary approach is optimistic for all the test grids as compared to original approach. The average error in estimation is found to be $19.6 \%$. Intuitively, $\varepsilon^{\prime}$ should be proportional to both $v_{\text {margin }}=v_{t h}-\max \left(v_{0}\right)$ and $\sigma_{\ln }$, because $v_{\text {margin }}$ and $\sigma_{\ln }$ control the length and the re-ordering in the sequence of interconnect failures because of TTF updates in a given realization. If these parameters are small, the error should be small. Figure 4.6 clearly confirms this intuition. 
Table 4.2: Comparision of Power Grid MTF $\mu_{s}$ and $\mu_{m}^{*}$ as obtained using the Series model and Mesh model for the original approach. $\mu_{s}$ and $\mu_{m}^{*}$ are estimated with $95 \%$ confidence and maximum $5 \%$ relative error.

\begin{tabular}{|c|c|c|c|c|c|c|c|c|c|c|}
\hline \multirow{2}{*}{$\begin{array}{c}\text { Grid } \\
\text { Name }\end{array}$} & \multirow{2}{*}{$\begin{array}{c}\max \left(v_{0}\right) \\
\left(\% v_{d d}\right)\end{array}$} & \multicolumn{2}{|c|}{ Series Model } & \multicolumn{5}{|c|}{ Mesh Model with TTF updates turned on } & \multirow{2}{*}{$\begin{array}{c}\text { Gain } \\
\left(\mu_{m}^{*} / \mu_{s}\right)\end{array}$} & \multirow{2}{*}{$\begin{array}{c}\text { CPU } \\
\text { Time } \\
t_{c p u}\end{array}$} \\
\hline & & $\begin{array}{c}\mu_{s} \\
(\mathrm{yrs}) \\
\end{array}$ & $\begin{array}{c}\# \mathrm{MC} \\
w_{s}\end{array}$ & $\begin{array}{c}\mu_{m}^{*} \\
(\mathrm{yrs})\end{array}$ & $\begin{array}{c}\# \mathrm{MC} \\
w_{m}\end{array}$ & $\begin{array}{c}\text { \#Fails } \\
\overline{k_{f}}\end{array}$ & $\begin{array}{c}\text { Time/Fail } \\
\overline{t_{f}}\end{array}$ & $\begin{array}{c}\text { Time/MC } \\
\overline{t_{m c}}\end{array}$ & & \\
\hline IBMPG2 & $7.0 \%$ & 10.18 & 183 & 34.06 & 50 & 20.22 & $0.29 \mathrm{~s}$ & $5.96 \mathrm{~s}$ & 3.35 & $4.97 \mathrm{~m}$ \\
\hline IBMPG3 & $6.8 \%$ & 9.48 & 161 & 40.01 & 86 & 46.70 & $3.04 \mathrm{~s}$ & $2.36 \mathrm{~m}$ & 4.22 & $3.39 \mathrm{~h}$ \\
\hline IBMPG4 & $7.4 \%$ & 10.28 & 122 & 37.65 & 51 & 41.63 & $3.83 \mathrm{~s}$ & $2.65 \mathrm{~m}$ & 3.66 & $2.26 \mathrm{~h}$ \\
\hline IBMPG5 & $4.8 \%$ & 9.96 & 193 & 32.34 & 89 & 17.27 & $1.59 \mathrm{~s}$ & $27.49 \mathrm{~s}$ & 3.25 & $40.78 \mathrm{~m}$ \\
\hline IBMPG6 & $5.7 \%$ & 10.47 & 171 & 41.93 & 76 & 32.76 & $3.28 \mathrm{~s}$ & $1.79 \mathrm{~m}$ & 4.01 & $2.27 \mathrm{~h}$ \\
\hline IBMPGNEW1 & $9.4 \%$ & 12.94 & 172 & 59.96 & 92 & 60.8 & $2.76 \mathrm{~s}$ & $2.8 \mathrm{~m}$ & 4.64 & $4.29 \mathrm{~h}$ \\
\hline IBMPGNEW2 & $7.7 \%$ & 10.12 & 164 & 39.64 & 75 & 41.97 & $5.35 \mathrm{~s}$ & $3.74 \mathrm{~m}$ & 3.92 & $4.68 \mathrm{~h}$ \\
\hline G1 & $3.6 \%$ & 10.12 & 186 & 33.64 & 87 & 24.14 & $0.21 \mathrm{~s}$ & $5.07 \mathrm{~s}$ & 3.32 & $7.35 \mathrm{~m}$ \\
\hline G2 & $4.6 \%$ & 10.27 & 132 & 33.7 & 76 & 34.59 & $0.55 \mathrm{~s}$ & $19.16 \mathrm{~s}$ & 3.28 & $24.27 \mathrm{~m}$ \\
\hline G3 & $5.0 \%$ & 10.89 & 115 & 37.87 & 82 & 51.02 & $1.10 \mathrm{~s}$ & $56.11 \mathrm{~s}$ & 3.48 & $1.28 \mathrm{~h}$ \\
\hline G4 & $4.6 \%$ & 12.03 & 121 & 49.92 & 56 & 97.21 & $3.02 \mathrm{~s}$ & $4.89 \mathrm{~m}$ & 4.15 & $4.56 \mathrm{~h}$ \\
\hline G5 & $4.0 \%$ & 10.48 & 86 & 42.90 & 50 & 115.2 & $5.86 \mathrm{~s}$ & $11.25 \mathrm{~m}$ & 4.09 & $9.37 \mathrm{~h}$ \\
\hline \multicolumn{9}{|c|}{ Average Gain ratio } & 3.78 & \\
\hline
\end{tabular}


Table 4.3: Speed and accuracy of the secondary approach, $\mu_{m}^{\prime}$ is estimated with $95 \%$ confidence and maximum $5 \%$ relative error.

\begin{tabular}{|c|c|c|c|c|c|c|c|c|c|c|}
\hline \multirow{3}{*}{$\begin{array}{l}\text { Grid } \\
\text { Name }\end{array}$} & \multirow{3}{*}{$\begin{array}{l}\max \left(v_{0}\right) \\
\left(\% v_{d d}\right)\end{array}$} & \multicolumn{6}{|c|}{ Mesh Model with TTF updates turned off } & \multicolumn{3}{|c|}{ Comparision } \\
\hline & & \multirow{2}{*}{$\begin{array}{c}\mu_{m}^{\prime} \\
(\mathrm{yrs})\end{array}$} & \multirow{2}{*}{$\begin{array}{c}\# \mathrm{MC} \\
w_{m} \\
\end{array}$} & \multirow{2}{*}{$\begin{array}{c}\text { \#Fails } \\
\overline{k_{f}}\end{array}$} & \multirow{2}{*}{$\begin{array}{c}\text { Time/Fail } \\
\overline{t_{f}}\end{array}$} & \multirow{2}{*}{$\begin{array}{c}\text { Time/MC } \\
\overline{t_{m c}}\end{array}$} & \multirow{2}{*}{$\begin{array}{c}\text { CPU-Time } \\
t_{c p u}\end{array}$} & \multirow[t]{2}{*}{$\varepsilon^{\prime}$} & \multicolumn{2}{|c|}{ Speed-up in } \\
\hline & & & & & & & & & $\overline{t_{f}}$ & $t_{c p u}$ \\
\hline IBMPG2 & $7.0 \%$ & 35.72 & 64 & 19.50 & $0.15 \mathrm{~s}$ & $3.01 \mathrm{~s}$ & $3.21 \mathrm{~m}$ & $\uparrow 4.88 \%$ & $1.91 \mathrm{x}$ & $1.54 \mathrm{x}$ \\
\hline IBMPG3 & $6.8 \%$ & 44.97 & 89 & 51.73 & $1.51 \mathrm{~s}$ & $1.30 \mathrm{~m}$ & $1.93 \mathrm{~h}$ & $\uparrow 12.39 \%$ & $2.01 \mathrm{x}$ & $1.75 \mathrm{x}$ \\
\hline IBMPG4 & $7.4 \%$ & 40.74 & 53 & 44.72 & $2.40 \mathrm{~s}$ & $1.79 \mathrm{~m}$ & $1.58 \mathrm{~h}$ & $\uparrow 8.21 \%$ & $1.6 \mathrm{x}$ & $1.43 \mathrm{x}$ \\
\hline IBMPG5 & $4.8 \%$ & 34.93 & 141 & 18.05 & $0.61 \mathrm{~s}$ & $10.94 \mathrm{~s}$ & $25.72 \mathrm{~m}$ & $\uparrow 8.00 \%$ & $2.63 \mathrm{x}$ & $1.59 \mathrm{x}$ \\
\hline IBMPG6 & $5.7 \%$ & 48.96 & 96 & 35.92 & $1.11 \mathrm{~s}$ & $39.78 \mathrm{~s}$ & $63.65 \mathrm{~m}$ & $\uparrow 16.77 \%$ & $2.97 \mathrm{x}$ & $2.14 \mathrm{x}$ \\
\hline IBMPGNEW1 & $9.4 \%$ & 60.97 & 93 & 64.53 & $1.20 \mathrm{~s}$ & $1.30 \mathrm{~m}$ & $2.01 \mathrm{~h}$ & $\uparrow 1.69 \%$ & $2.29 \mathrm{x}$ & $2.14 \mathrm{x}$ \\
\hline IBMPGNEW2 & $7.7 \%$ & 44.00 & 103 & 47.41 & $3.80 \mathrm{~s}$ & $3.00 \mathrm{~m}$ & $5.15 \mathrm{~h}$ & $\uparrow 11.00 \%$ & $1.41 \mathrm{x}$ & $0.91 \mathrm{x}$ \\
\hline G1 & $3.6 \%$ & 50.13 & 154 & 53.31 & $0.11 \mathrm{~s}$ & $6.05 \mathrm{~s}$ & $15.54 \mathrm{~m}$ & $\uparrow 49.04 \%$ & $1.85 \mathrm{x}$ & $0.47 \mathrm{x}$ \\
\hline G2 & $4.6 \%$ & 46.16 & 110 & 71.09 & $0.32 \mathrm{~s}$ & $22.48 \mathrm{~s}$ & $41.21 \mathrm{~m}$ & $\uparrow 36.97 \%$ & $1.75 \mathrm{x}$ & $0.59 \mathrm{x}$ \\
\hline G3 & $5.0 \%$ & 44.86 & 106 & 78.99 & $0.62 \mathrm{~s}$ & $48.72 \mathrm{~s}$ & $1.43 \mathrm{~h}$ & $\uparrow 18.45 \%$ & $1.78 \mathrm{x}$ & $0.89 \mathrm{x}$ \\
\hline G4 & $4.6 \%$ & 64.10 & 94 & 185.6 & $1.75 \mathrm{~s}$ & $5.40 \mathrm{~m}$ & $8.46 \mathrm{~h}$ & $\uparrow 28.41 \%$ & $1.73 \mathrm{x}$ & $0.54 \mathrm{x}$ \\
\hline G5 & $4.0 \%$ & 59.67 & 59 & 269.9 & $3.64 \mathrm{~s}$ & $16.39 \mathrm{~m}$ & $16.12 \mathrm{~h}$ & $\uparrow 39.08 \%$ & $1.61 \mathrm{x}$ & $0.58 \mathrm{x}$ \\
\hline \multicolumn{8}{|c|}{ Average Relative error and speed-up } & $19.57 \%$ & $1.96 \mathrm{x}$ & $1.21 \mathrm{x}$ \\
\hline
\end{tabular}




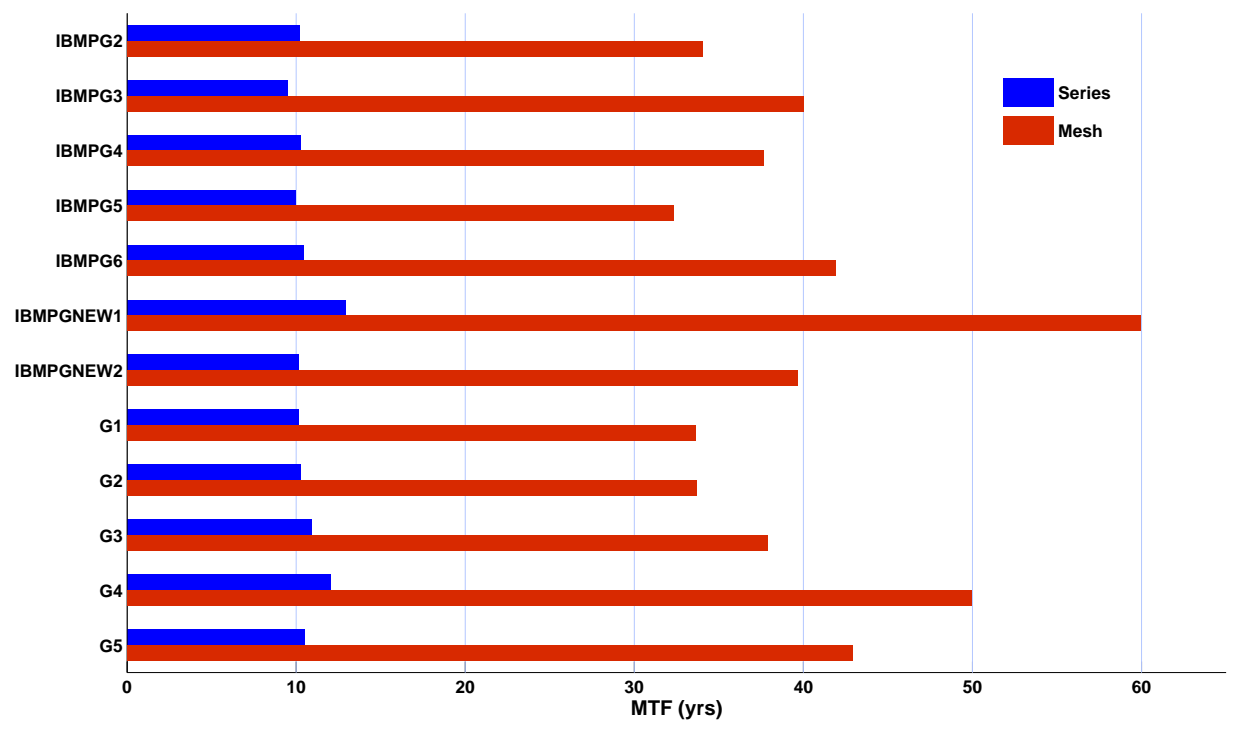

Figure 4.5: Comparison of MTF as obtained using the series model and mesh model for the original approach

When TTF updates are turned off, no computation power is spent to update the statistics of the surviving interconnects. Figure 4.7 shows the average division of CPU time between voltage updates and TTF updates for each grid after an interconnect failure as observed from the original approach. Hence, by eliminating the TTF updates in secondary approach, there is an average speed-up of $1.96 \mathrm{x}$ in $\overline{t_{f}}$. However, eliminating TTF updates slows down the convergence of Monte Carlo iterations and increases number of interconnect failures required to fail a grid. In effect, this increases $w_{m}$ and $\overline{k_{f}}$. From (4.27), we have (the subscript org stands for the original approach and sec stands for the secondary approach):

$$
\frac{t_{c p u, \text { org }}}{t_{c p u, s e c}}=\frac{w_{m, \text { org }} \times \overline{t_{f, \text { org }}} \times \overline{k_{f, \text { org }}}}{w_{m, \text { sec }} \times \overline{t_{f, s e c}} \times \overline{k_{f, s e c}}}
$$

Thus, for the secondary approach, if the speed-up obtained in $\overline{t_{f}}$ is offset by slow Monte Carlo convergence and longer failure sequences, the net CPU time $t_{c p u}$ required to estimate grid MTF increases, otherwise it decreases. For the test grids, an average speed-up of $1.2 \mathrm{x}$ was observed in $t_{c p u}$ when TTF updates are turned off, which is very less as compared to the speed-up obtained for $\overline{t_{f}}$.

To conclude, the TTF update framework presented in this chapter is important because it removes the optimism that creeps in when the effect of failing interconnects on the surviving ones are not considered and improves the convergence. It acknowledges that the failure sequence of interconnects is dynamic, in that it can change based on the history of failures up to the present time. However, TTF updates can be turned off especially when $\max \left(v_{0}\right)$ is close to $v_{t h}$ and $\sigma_{\ln }$ is small, since $\varepsilon^{\prime}$ in this case will be small and convergence is not an issue. 

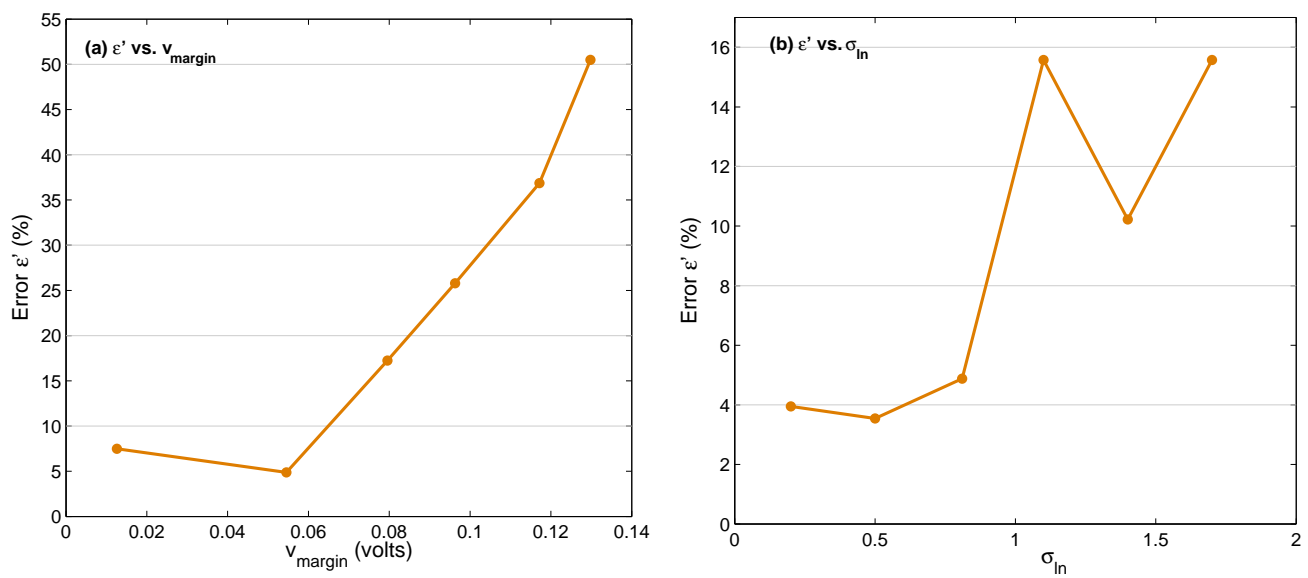

Figure 4.6: Dependence of relative error between $\mu_{m}^{\prime}$ and $\mu_{m}^{*}$ on $\left(v_{t h}-\max \left(v_{0}\right)\right)$ and $\sigma_{\ln }$ for IBMPG2 grid

Scalability The problem size in our case is decided by the sum of number of nodes $m$ and number of interconnects branches $b$ in the power grid, because we update the node voltage drops and the statistics of the surviving branches for each failure. However, $b=\mathcal{O}(m)$. Hence, we use $m$ as the problem size in scalability analysis. We determine the scalability of the following for the original approach a) Average time taken to update voltage drops and statistics $\left.\left(\overline{t_{f}}\right), \mathrm{b}\right)$ Average time per Monte Carlo iteration $\left(\overline{t_{m c}}\right)$, c) Total time taken to estimate MTF $\left.\left(t_{c p u}\right) \mathrm{d}\right)$ Maximum memory used. As shown in figure 4.8, scalability of $\overline{t_{f}}, \overline{t_{m c}}$ and $t_{c p u}$ are found to be $\mathcal{O}\left(m^{1.19}\right), \mathcal{O}\left(m^{1.61}\right)$ and $\mathcal{O}\left(m^{1.55}\right)$, respectively. The space complexity is found to $\mathcal{O}\left(m^{1.12}\right)$.

Voltage maps Finally, we plot a voltage-drop map of three test grids at $t=0$ (when they are fresh), and when they fail as per the mesh model. The maps clearly indicate the hot regions on the grid, which may be used as a graphical feedback to the user for further improving the design of the power grid. 


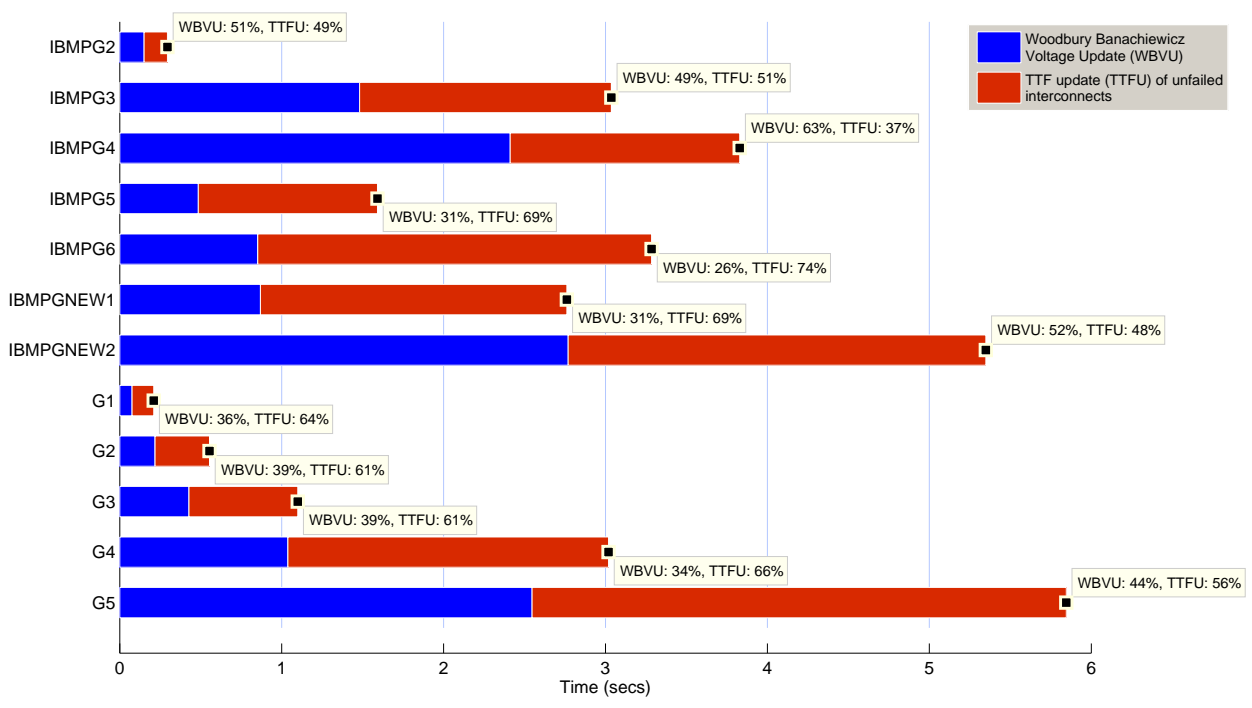

Figure 4.7: Average time-breakup between node voltage updates and TTF updates of surviving interconnects after an interconnect failure
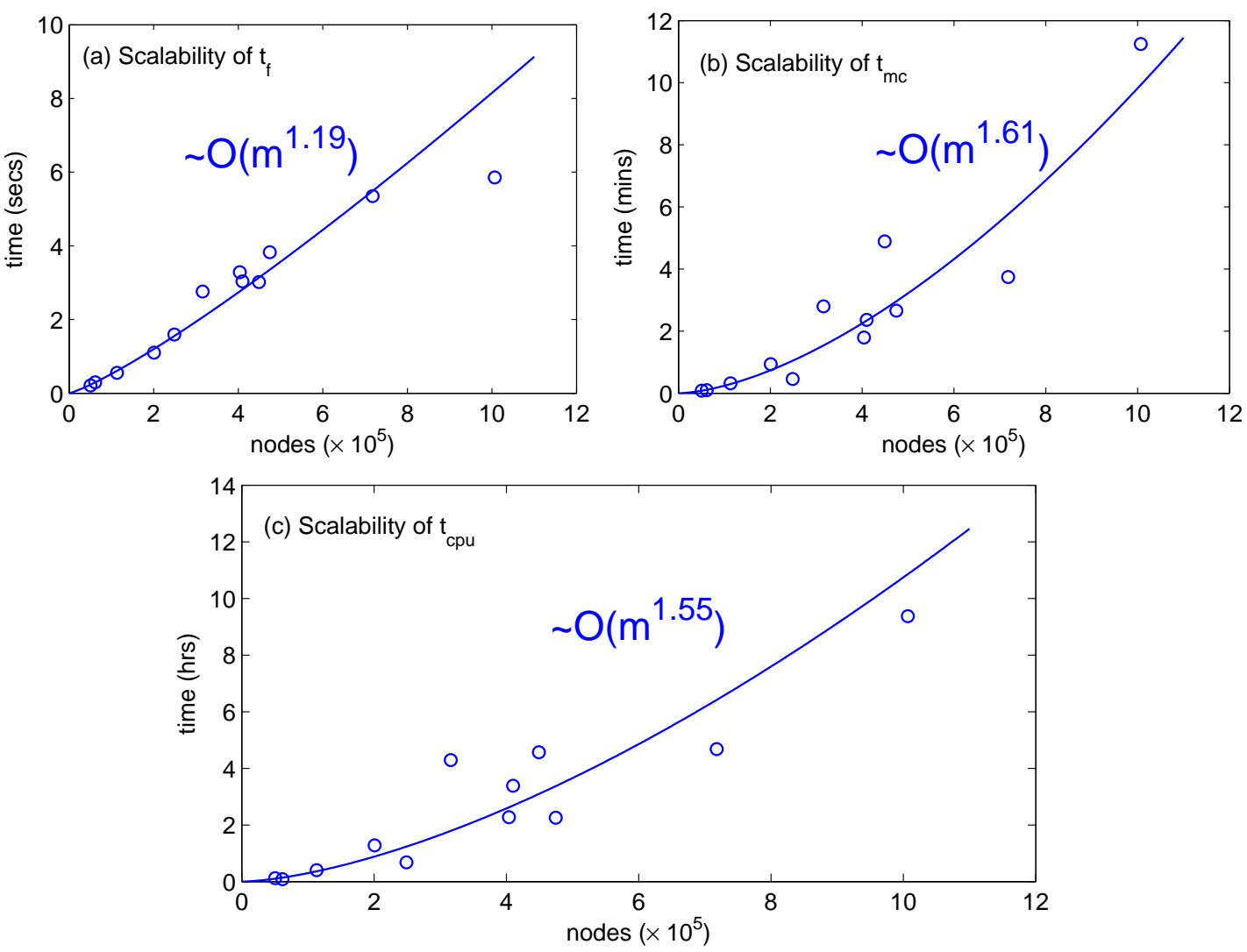

Figure 4.8: Scalability of the original approach 


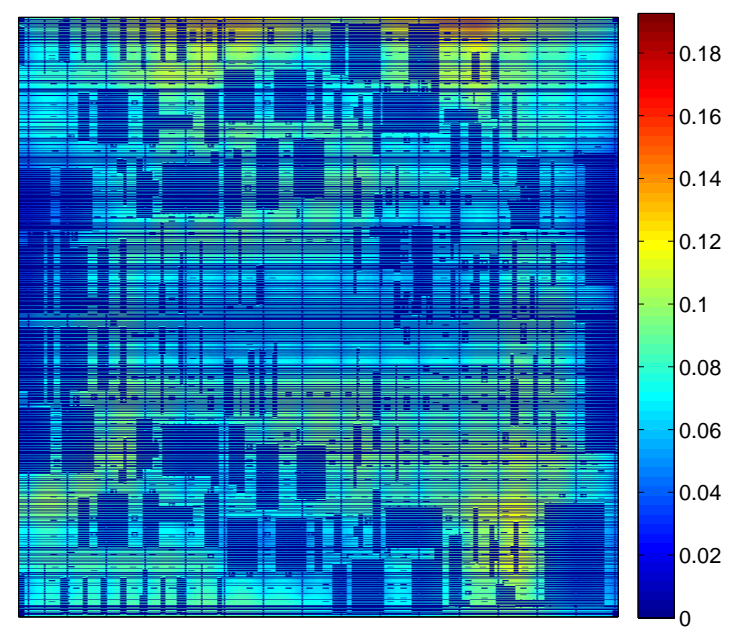

(a)

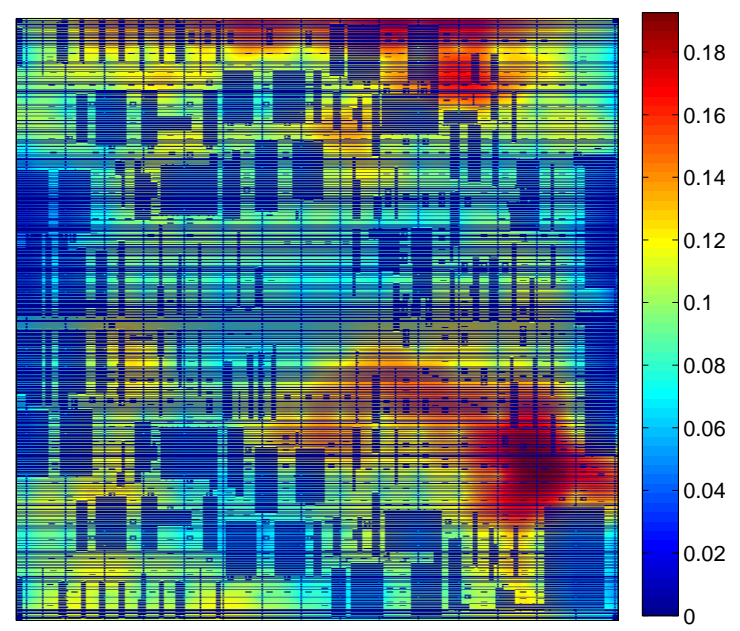

(b)

Figure 4.9: Voltage drop maps of IBMPGNEW2 (a) at $t=0$ and (b) at the time of failure grid failure. For this particular realization, 68 interconnect failures were required to exceed threshold voltage $v_{t h}=0.18 \mathrm{~V}$

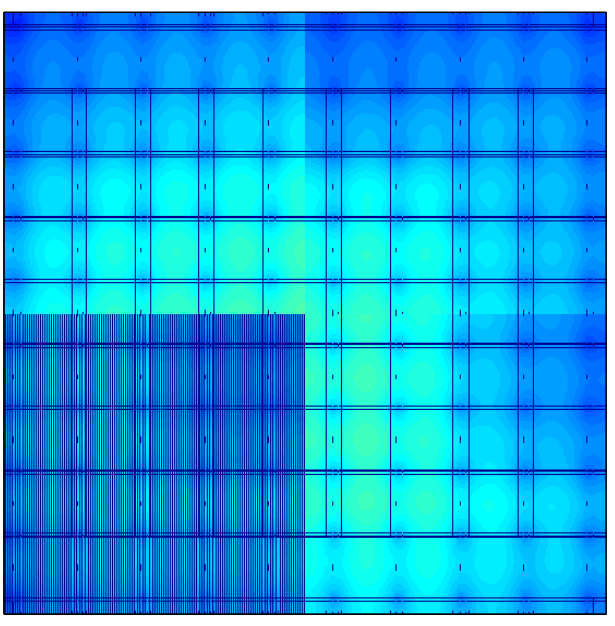

(a)
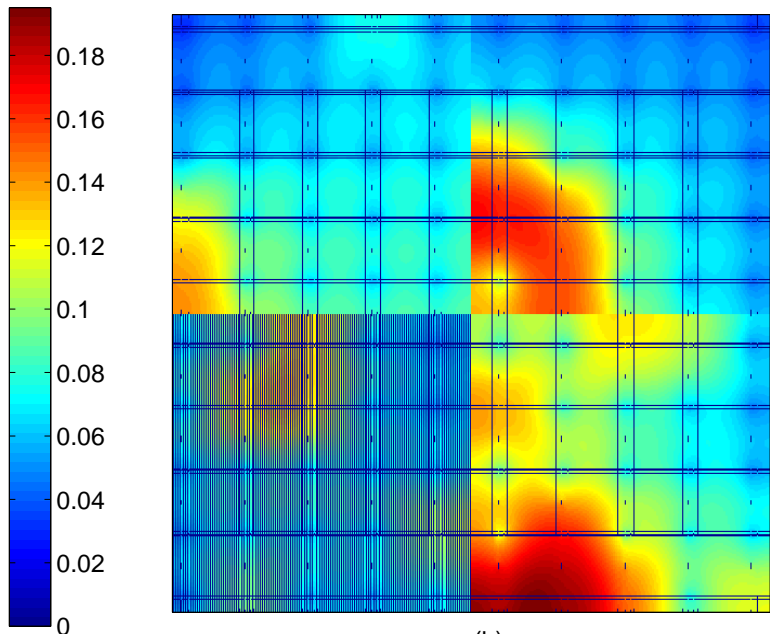

(b)

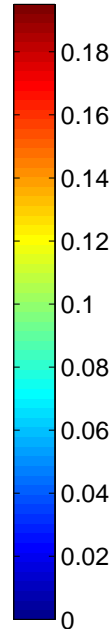

0

Figure 4.10: Voltage drop maps of IBMPG5 (a) at $t=0$ and (b) at the time of failure grid failure. For this particular realization, 20 interconnect failures were required to exceed threshold voltage $v_{t h}=0.18 \mathrm{~V}$ 


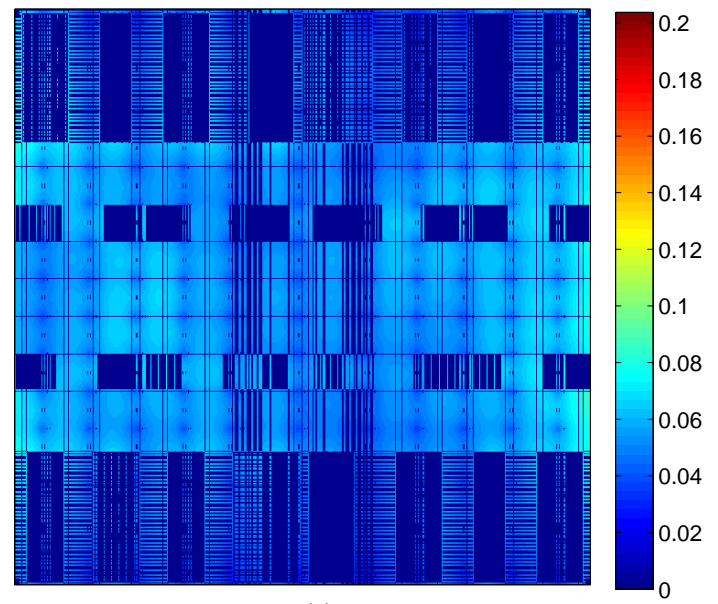

(a)

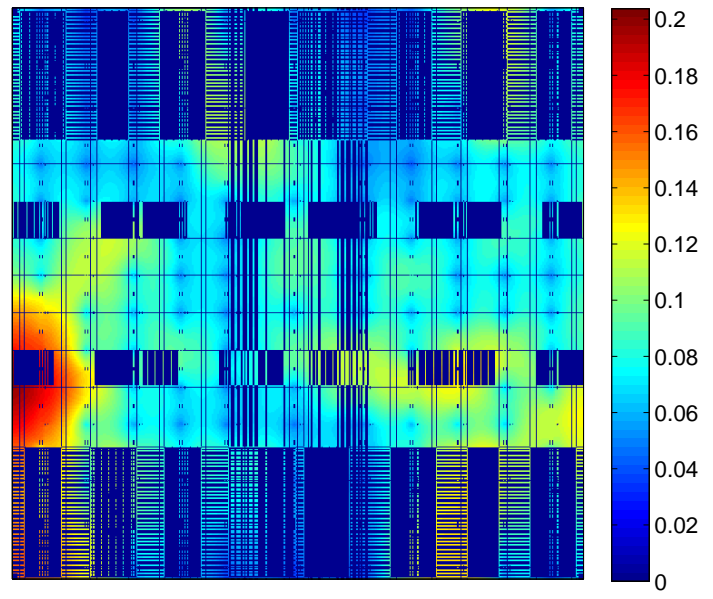

(b)

Figure 4.11: Voltage drop maps of IBMPG6 (a) at $t=0$ and (b) at the time of failure grid failure. For this particular realization, 36 interconnect failures were required to exceed threshold voltage $v_{t h}=0.18 \mathrm{~V}$ 


\section{Chapter 5}

\section{Reliability Estimation and Selective TTF updates}

\subsection{Introduction}

In this chapter, we first develop a framework to estimate the probability of survival of a power grid for a given period of time based on Monte Carlo random sampling approach as per the series and the mesh model. This framework enables us to perform a complete statistical analysis of the power grid as per the series and the mesh model, and draw important conclusions about the statistical nature of grid failure. Next, we describe a heuristic approach to speedup the MTF estimation engine by performing selective TTF updates, i.e updating the statistics of only significantly impacted interconnects for a given interconnect failure. The results show $\mathrm{a} \sim 2 \mathrm{x}$ speedup with negligible loss in accuracy. Finally, it is shown that the reduction in pessimism as predicted by the mesh model is stable.

\subsection{Survival Probability and Reliability Estimation}

Sometimes, users are interested in finding the probability of survival of a given grid for a period of $y$ years. This is called Survival Probability Estimation of the grid for a time-period of $y$ years, denoted by $S P E(y)$. In order to estimate the probability of survival of a given grid up to a period of $y$ years, Monte Carlo random sampling is used, the details of which are given in section 2.5.2. The stopping criterion (in terms of number of samples $w$ required) is defined such that there is $(1-\alpha) \times 100 \%$ confidence that the error in probability estimation is less than $\mathcal{E}[36]:$ 


$$
w=\max \left[\left(\frac{z_{\alpha / 2}}{2 \mathcal{E}}\right)^{2},\left(\frac{\sqrt{63}+z_{\alpha / 2}}{2 \sqrt{\mathcal{E}}}\right)^{2},\left(\frac{z_{\alpha / 2} \sqrt{2 \mathcal{E}+0.1}+\sqrt{(\mathcal{E}+0.1) z_{\alpha / 2}^{2}+3 \mathcal{E}}}{2 \mathcal{E}}\right)^{2}\right]
$$

where $z_{\alpha / 2}$ is as defined in section 2.5.1.

SPE Algorithm Algorithm 4 estimates the survival probability of a given grid for a time period of $y$ years as per both the series and mesh models using the process of Bernoulli trials: for each trial (iteration), we have a success if the grid survives for $y$ years, or else we have a failure. If $x$ successes were observed in $w$ trials, the survival probability is estimated to be $\frac{x}{w}$.

Apart from $y$, the other inputs are the conductance matrix of the fresh grid $G_{0}$, the vector of current sources $i$, the list of all interconnects in the grid $\left(\mathrm{R}_{\text {list }}\right)$, the vector of initial voltage drops at $t=0\left(v_{0}\right)$, the voltage drop threshold vector for all nodes of the grid $\left(v_{t h}\right)$, the critical Blech product $\left(\beta_{c}\right)$ and a flag enable_ttf_update which controls if the TTF updates are to be performed while obtaining grid TTF sample. The outputs are $\mathcal{P}_{s}=\mathcal{P}\left(\tau_{s} \geq y\right)$ (probability of grid surviving more than $y$ yrs using the series system model) and $\mathcal{P}_{m}=\mathcal{P}\left(\tau_{m} \geq y\right)$ (probability of grid surviving more than $y$ yrs using the mesh system model). Both $\mathcal{P}_{s}$ and $\mathcal{P}_{m}$ are estimated with a confidence of $95 \%(\alpha=0.05)$ and a maximum relative error of $5 \%(\mathcal{E}=0.05)$, which leads to a stopping criteria of $w=490$ iterations, as per (5.1).

Reliability Estimation The reliability of a power grid is essentially its survival probability at different points in time. Hence, if the statistics of TTFs of the power grid following the mesh and series model is described by the RVs $\mathbf{T}_{m}$ and $\mathbf{T}_{s}$, then their probability distribution function and reliability can be calculated empirically by obtaining $w$ sample grid TTF values as per the respective models and calculating the survival probabilities for different values of $y$.

It is useful in two ways:

- Quick analysis: If the stopping criterion is relaxed (by increasing $\alpha$ and $\mathcal{E}$ ), one can quickly verify if the given grid still satisfies the target MTF $\mu$ (by ensuring $\mathcal{P}_{m}-\mathcal{E}>0.5$ at $y=\mu$ ). This is especially useful when the power grid design goes through incremental changes, and it is costly to run a full-fledged MTF estimation engine for every change.

- Complete Statistical Analysis: On the other hand, towards the end of grid design, a designer can use Algorithm 4 with a strict stopping criteria to get complete information about RVs $\mathbf{T}_{m}$ and $\mathbf{T}_{s}$, in the form of their CDF or Reliability function. A complete statistical analysis for grid IBMPG5 is presented later in this chapter. 


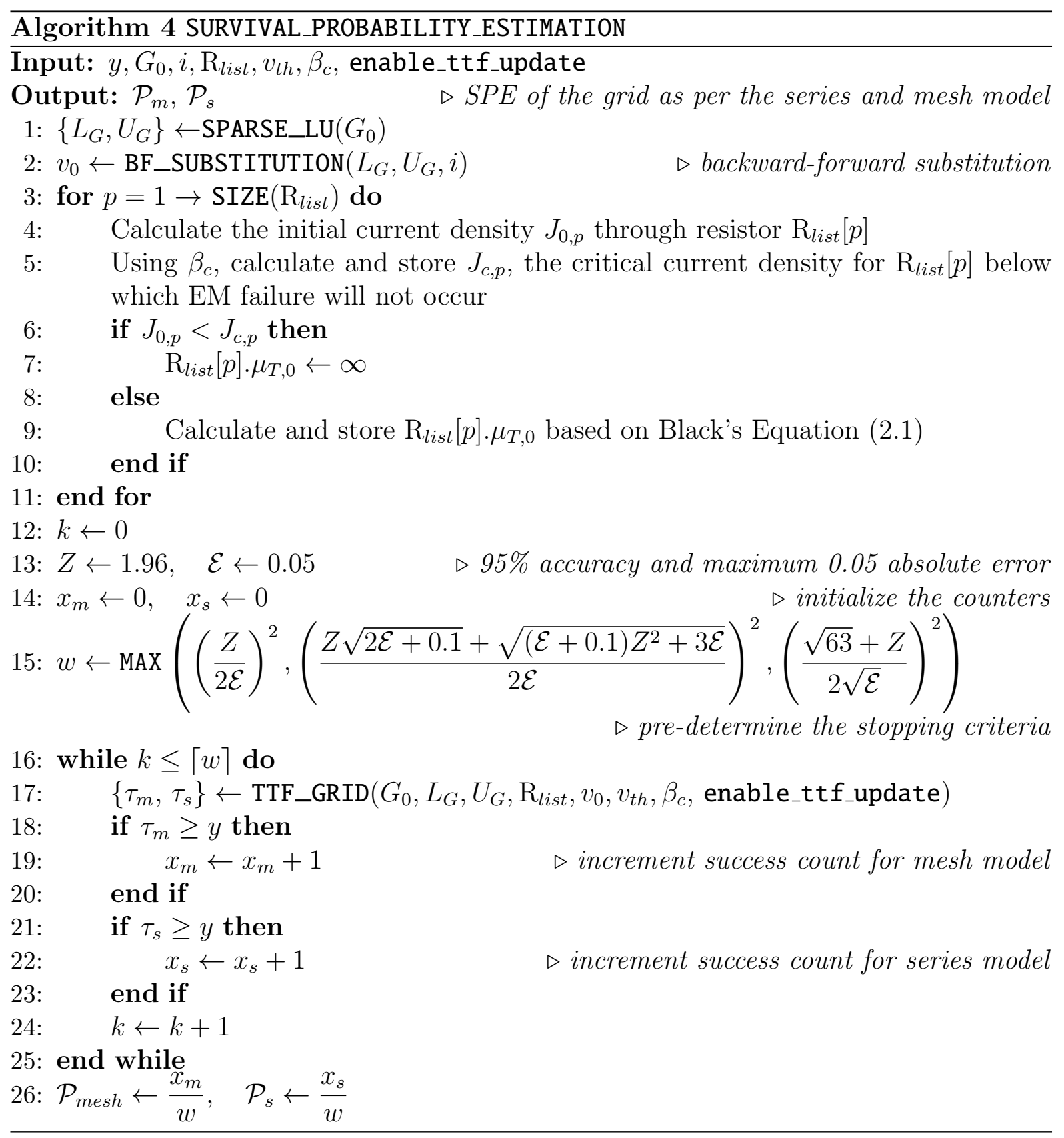




\subsection{Selective TTF updates for speed-up}

\subsubsection{Motivation}

In MTF estimation and survival probability estimation of a power grid as per the mesh model, the bulk of the computation effort is spent on:

1. updating the voltage drop of nodes after an interconnect failure.

2. updating the TTFs of surviving interconnects to determine the next interconnect to fail.

Figure 4.7 (in chapter 4) shows for each grid, the computation time spent after each interconnect failure in updating voltage drops and interconnect TTFs. On average, $41.3 \%$ computation time is spent in updating voltage drops and $58.7 \%$ time is spent in updating TTFs. The secondary approach, where TTFs are not updated for any interconnect, sped-up the average time it takes to determine the next interconnect to fail $\left(\overline{t_{f}}\right)$. But, this speed-up was not carried over to total time $t_{c p u}$ due to slower convergence. The approach presented in this section is aimed at reducing the CPU time spent in updating the TTFs in a way that minimally affect the convergence. Another advantage of this approach is that it helps trade-off accuracy for speed, if required.

\subsubsection{Overview of approach}

When an interconnect in the power grid fails, not all the nodes are equally impacted. This is easily verified by figure 5.1, which shows the change in node voltage drops for a randomly chosen single interconnect failure in power grids IBMPG5, IBMPG6, IBMPGNEW2 and G4. The sensitivity of voltage drops due to interconnect failure is higher in external grids because these grids have comparatively large number of current sources distributed throughout the network. In any case, it is clearly visible that for a given interconnect failure, only a subset of the nodes (and the associated links), in the immediate neighborhood are significantly impacted. This locality effect can be exploited to update the TTFs of selective interconnects and thus, speed-up the estimation engine, at the cost of some loss in accuracy of estimation.

Let the set of all nodes be represented by $\mathcal{N}$. Each node in the power grid is represented in the set by its node number. Thus $\mathcal{N}$ is the set of integers from 1 to $m$, where $m$ is the number of nodes in the power grid:

$$
\mathcal{N}=\{1,2,3, \ldots m-1, m\}
$$

Also, let $\Gamma\left(\mathcal{N}_{\text {sub }}\right)$, where $\mathcal{N}_{\text {sub }} \subseteq \mathcal{N}$, be the set of all resistors connected to nodes $k \in \mathcal{N}_{\text {sub }}$. Note that $\Gamma(\mathcal{N})$ is the set of all resistors in the power grid. Then, if $\frac{\partial v_{k}}{\partial \mathrm{R}}$ is the change in the voltage drop of the $k^{\text {th }}$ node with respect to failure of interconnect $\mathrm{R}$, we define the region of impact of an interconnect $\mathrm{R}, \pi(\mathrm{R})$ to be: 

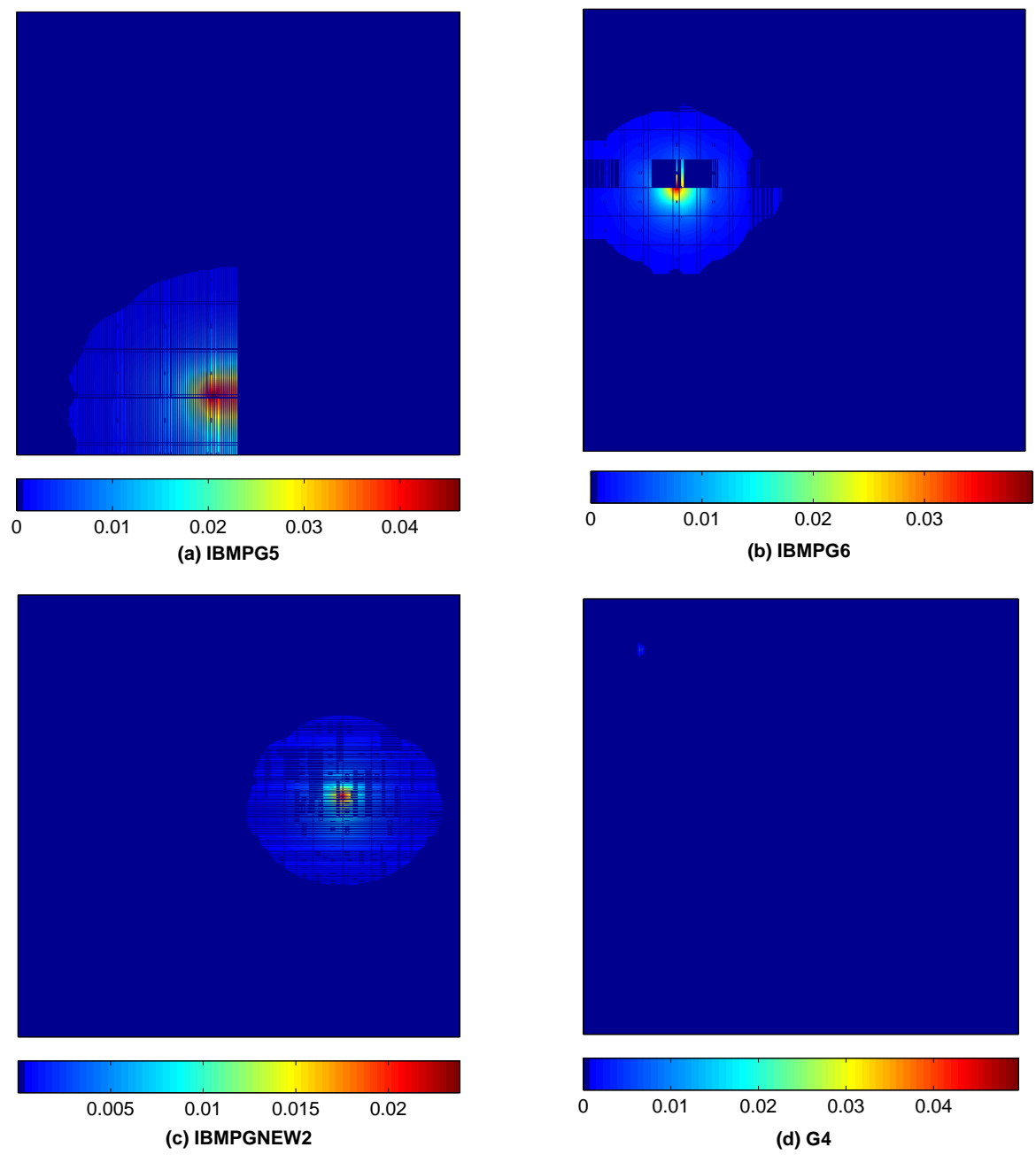

Figure 5.1: Change in node voltage drops for a randomly chosen single interconnect failure in power grids IBMPG5, IBMPG6, IBMPGNEW2 and G4. Nodes where change in voltage drop is less than $10^{-3}$ are omitted in the figure.

$$
\pi(\mathrm{R}) \triangleq \Gamma\left(\mathcal{N}_{\mathrm{R}}\right) \quad \text { such that } \quad \mathcal{N}_{\mathrm{R}}=\left\{k \in \mathcal{N}: \frac{\partial v_{k}}{\partial \mathrm{R}}>\delta_{v}^{\star}\right\}
$$

$\delta_{v}^{\star}$ is a user-input that determines the boundary of the region of impact on the power grid. Intuitively, smaller value of $\delta_{v}^{\star}$ increases $|\pi(\mathrm{R})|$, which improves the accuracy of estimated MTF or survival probability at the cost of reduction in speed, and vice-versa. Figure 5.2 shows how the boundary of $\pi(\mathrm{R})$ varies with $\delta_{v}^{\star}$. The TTF of a resistor connected to a node $k \in \pi(\mathrm{R})$ is updated if $\mathrm{R}$ fails, otherwise its TTF is not updated. The heuristic employed here aims to reduce the error in estimation by updating the TTFs of significantly impacted interconnects only, hoping that the sequence of interconnect failures for this realization is as close as possible to the actual one obtained when the TTFs of all interconnects are updated. 


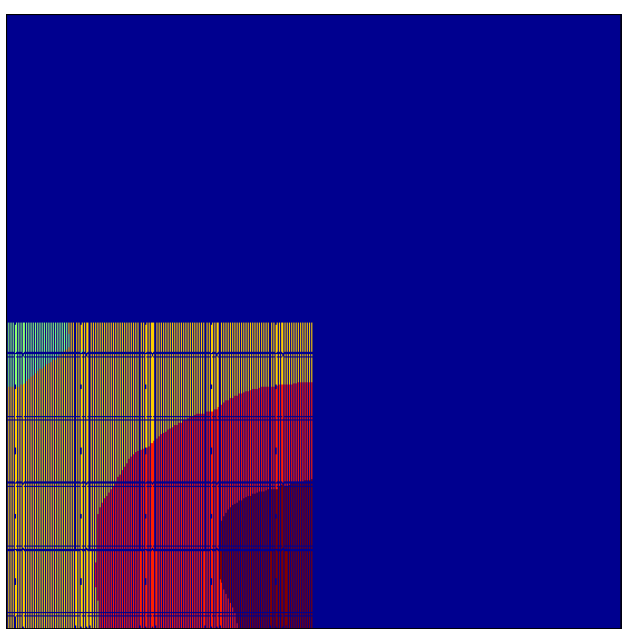

(a) IBMPG5

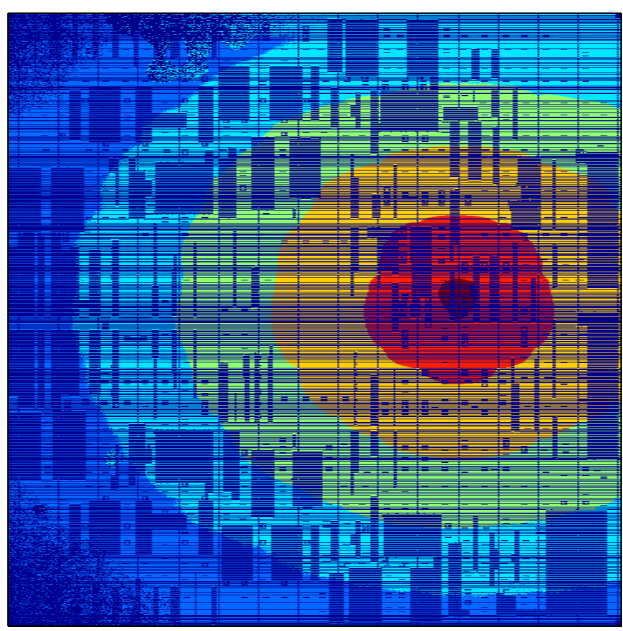

(c) IBMPGNEW2

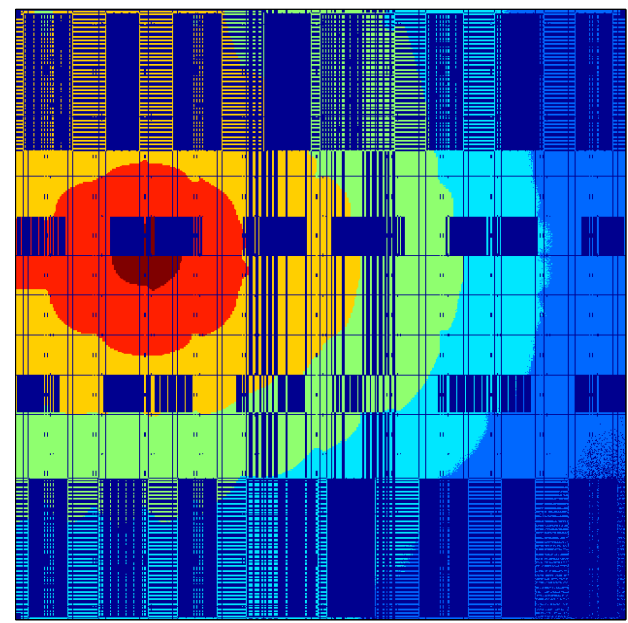

(b) IBMPG6

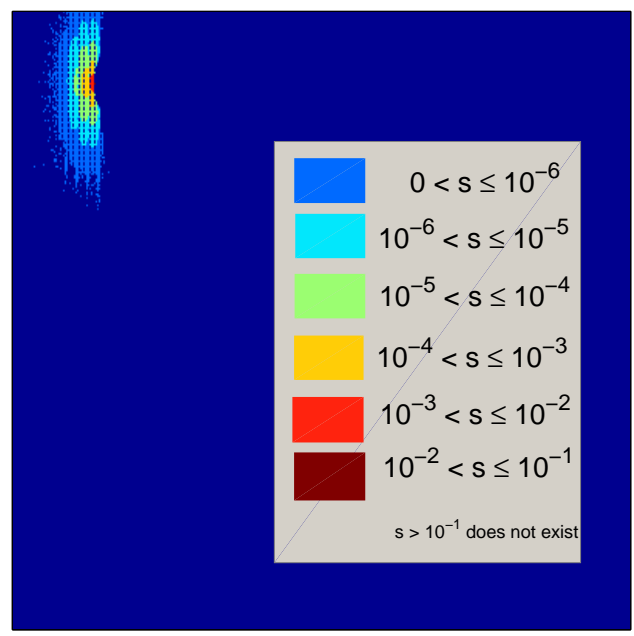

(d) G4

Figure 5.2: Dependence of boundary of $\pi(\mathrm{R})$ on $\delta_{v}^{\star}, s=\partial v_{k} / \partial \mathrm{R}$

The selective TTF update algorithm Algorithm 5 gives the implementation of the proposed selective updates approach. This algorithm is similar to the Algorithm 2 (in chapter 4), except in lines 18-20, where instead of updating the TTFs of all interconnects after a failure, only those interconnects that are in the set $\pi(\mathrm{R})$ for a given interconnect $\mathrm{R}$ are updated when $\mathrm{R}$ fails.

\subsection{Parameter Variation}

There are many factors that influence degradation of a metal line due to electromigration and thus affect the estimated MTF of the grid as per the series and mesh model. Some of these 


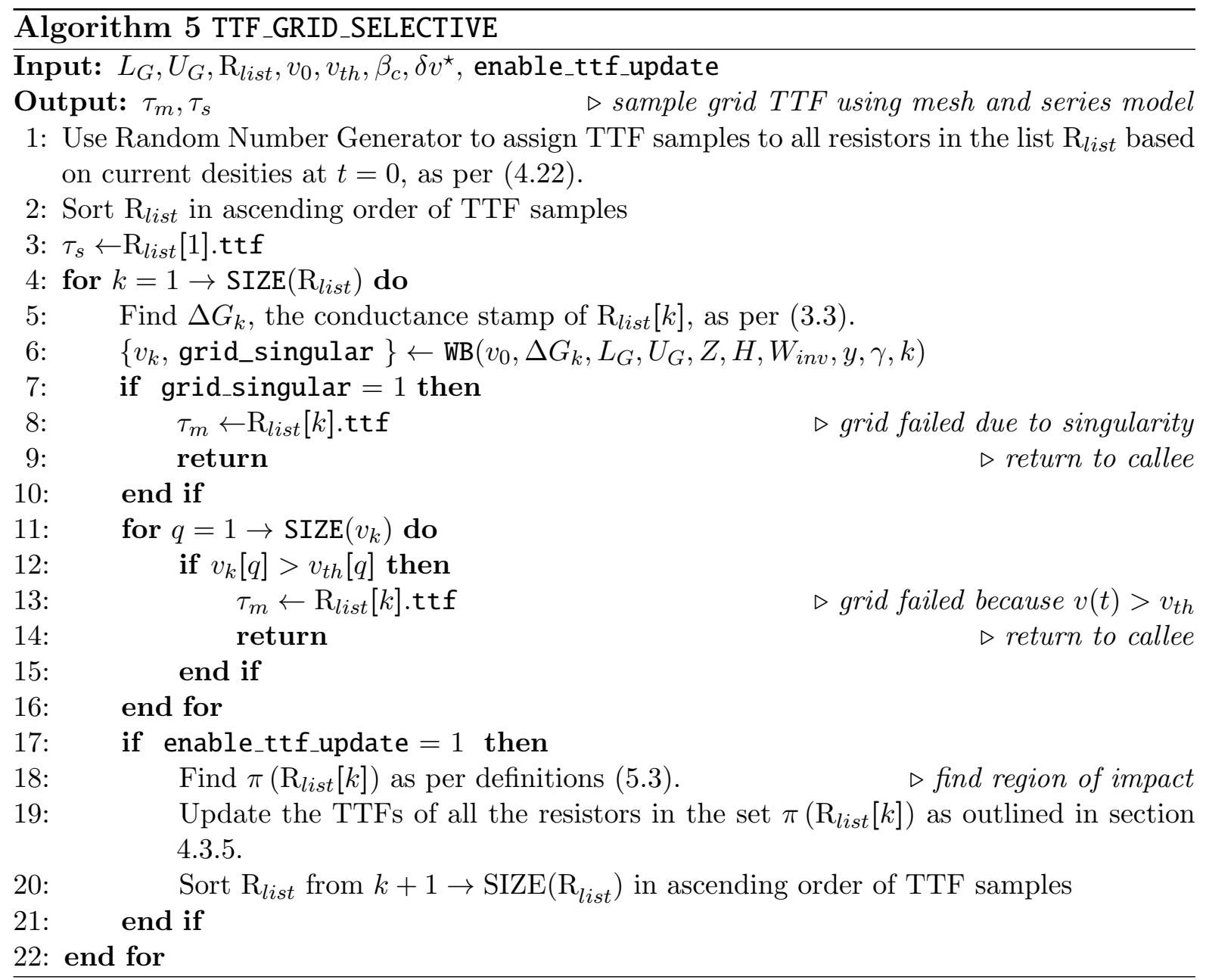

factors are:

1. power grid configuration, in terms of $\max \left(v_{0}\right)$ and metal temperature $T_{m}$.

2. property of metal used, in terms of Black's constant $A$, Activation Energy $E_{a}$, resistivity of metal $\rho$ and Blech product $\beta_{c}$.

3. choice of failure mechanism, in terms of current exponent $n$

4. effect of statistical parameters, quantified by standard deviation of lognormal $\sigma_{\ln }$

In all the experiments conducted so far, these factors/constants were given a specific value, for which the gain ratio was found to be 3-4 for all grids. However, the gain-ratio will be affected if the constants were given different values. In this section, we investigate the effect of varying these constants on the gain-ratio. The test grid used for this study is IBMPG2, but same conclusions can be drawn for other grids as well.

Figures 5.3, 5.4 and 5.5 depict the results of the experiments. Changing Black's constant $A$, Activation energy $E_{a}$ and Temperature of metal $T_{m}$ do not have any effect on the gain ratio 

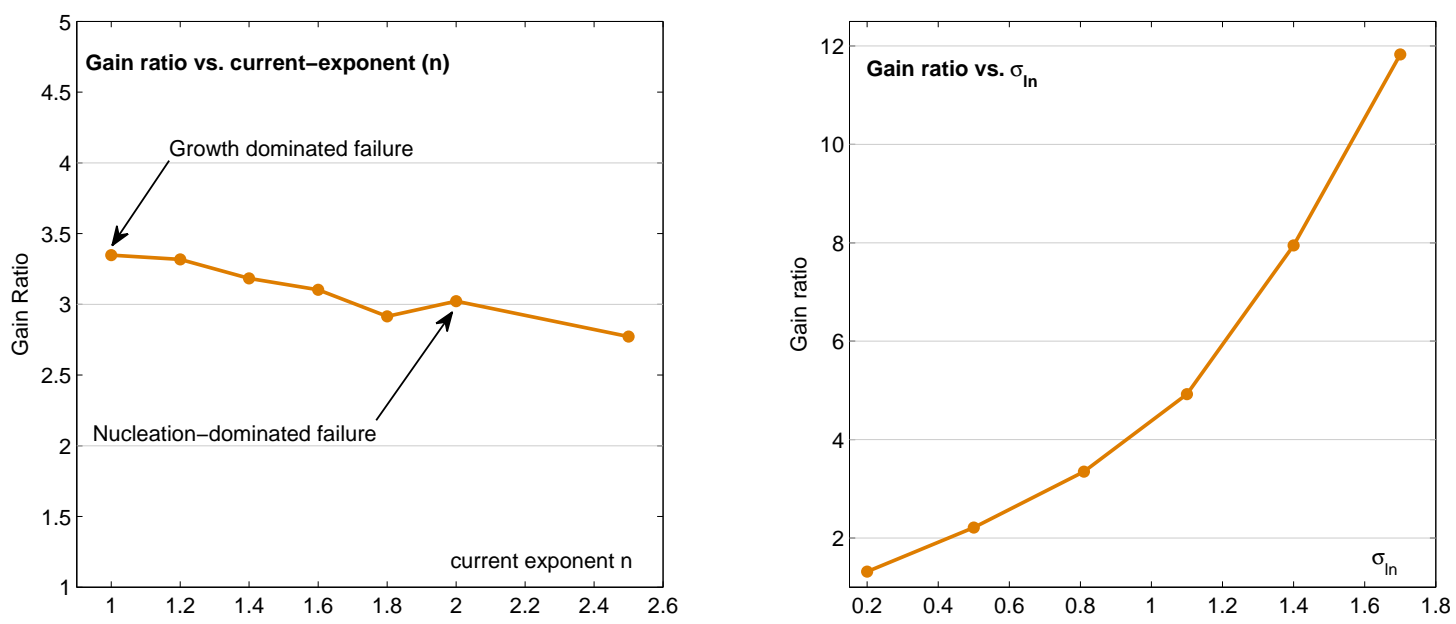

Figure 5.3: Change in gain ratio with current exponent $(n)$ and standard deviation of lognormal $\left(\sigma_{\ln }\right)$ for power grid IBMPG2

because if only $A, E_{a}$ and/or $T_{m}$ are changed and everything else is kept same, the TTFs of all interconnects are scaled linearly, which neither affects the sequence of failure of interconnects nor the ratio $\mu_{m} / \mu_{s}$. On the other hand, increase in standard deviation of lognormal, $\sigma_{\ln }$, increases the variance of TTFs, so that the difference in TTFs of consecutive interconnects in the failing sequence increases. Thus, the gain ratio increases. It is observed that with increasing current exponent $(n)$ the gain ratio decreases and with change in either resistivity $\rho$ of interconnects or Blech product $\beta_{c}$, the gain ratio fluctuates in a narrow range, but never plummets down drastically. This shows that the mesh model is robust and the increase in EM margin predicted using the mesh model is stable. Finally, as expected, the gain-ratio decreases with increase in $\max \left(v_{0}\right)$, since lesser and lesser interconnect failures are required to exceed $v_{t h}$.

\subsection{Implementation and Experimental Results}

The algorithms in section 5.2 and 5.3 have been implemented in C++. The experiments were carried out on both the internal and external grids, using a $2.6 \mathrm{GHz}$ Linux machine with 24 GB of RAM.

For Survival Probability Estimation, $y$ was a user-provided input. As mentioned before, $\mathcal{P}_{s}$ and $\mathcal{P}_{m}$ are estimated with a confidence of $95 \%(\alpha=0.05)$ and a maximum relative error of $5 \%(\mathcal{E}=0.05)$, which leads to a stopping criteria of $w=490$ iterations for all the test grids. However, this stopping criterion can be relaxed or made more strict, depending on the need of the user. Note that when only the survival probability of the grid for $y$ years is to be estimated, the actual implementation in C++ differs a little: algorithm TTF_GRID (or TTF_GRID_SELECTIVE) is modified so that it returns if the last failed interconnect had $\tau>y$ (which means that the grid will definitely survive beyond $y$ for this realization) or if the grid fails before $y$, whichever 

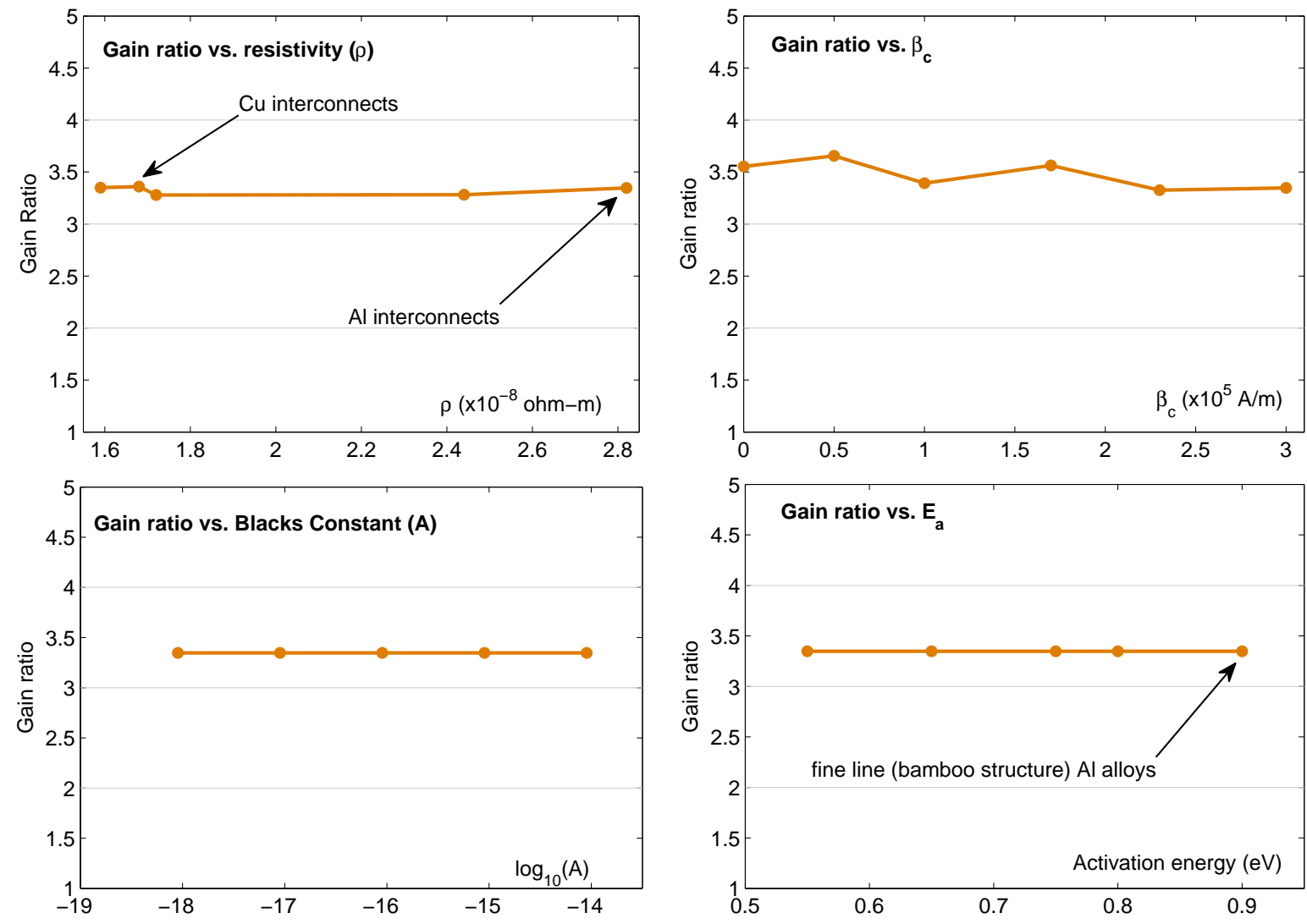

Figure 5.4: Variation of gain ratio with property of metal used for power grid IBMPG2
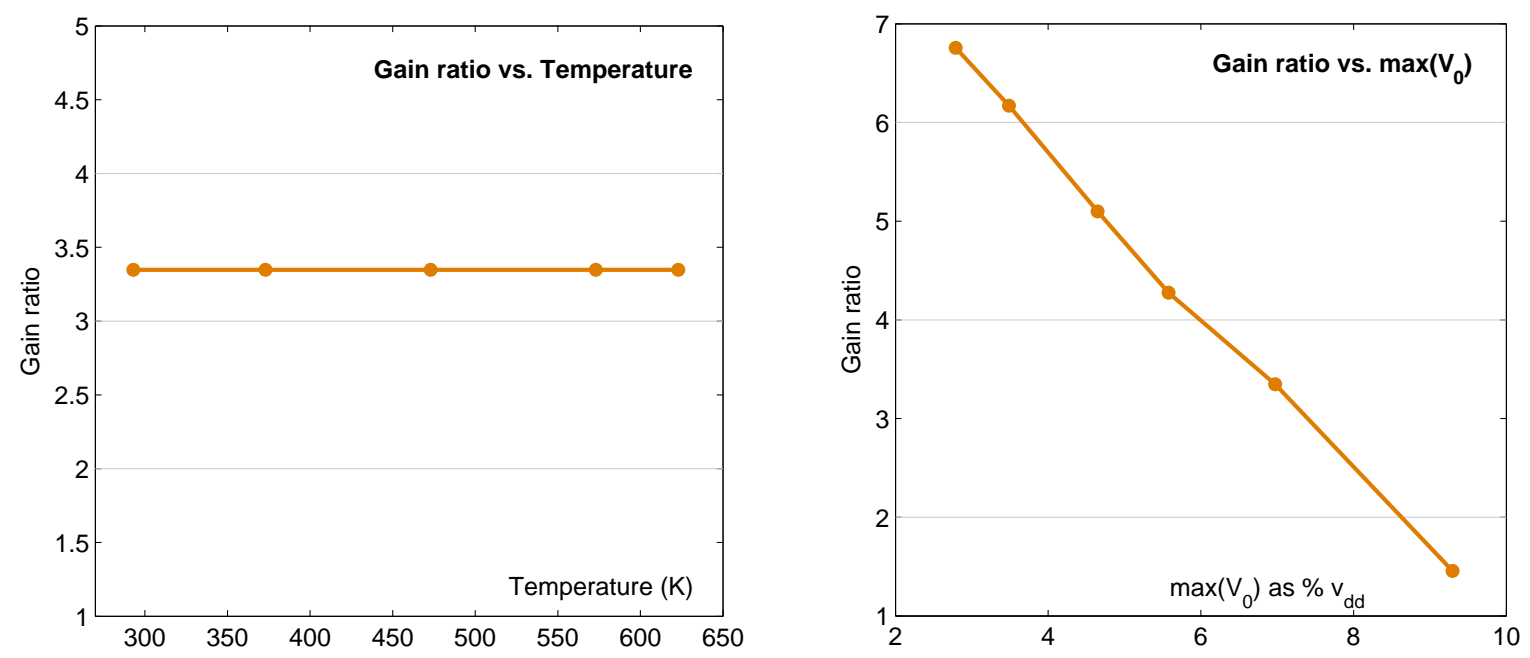

Figure 5.5: Variation of gain ratio with power grid configuration for power grid IBMPG2 


\begin{tabular}{|c|c|c|c|c|c|c|c|c|}
\hline \multirow{2}{*}{$\begin{array}{c}\text { Power } \\
\text { Grid }\end{array}$} & \multicolumn{2}{|c|}{$\max \left(v_{0}\right)$} & \multicolumn{3}{|c|}{$y$} & $\begin{array}{c}\mathcal{P}_{s} \\
\mathcal{P}\left(\tau_{s} \geq y\right)\end{array}$ & $\begin{array}{c}\mathcal{P}_{m} \\
\mathcal{P}\left(\tau_{m} \geq y\right)\end{array}$ & $\begin{array}{c}\text { CPU Time } \\
(\mathrm{s} / \mathrm{m} / \mathrm{h})\end{array}$ \\
\cline { 2 - 8 } volts & $\% v_{d d}$ & yrs & $y / \mu_{s}$ & $y / \mu_{m}^{*}$ & 1.000 & $7.32 \mathrm{~m}$ \\
\hline IBMPG2 & 0.125 & $7.0 \%$ & 12.8 & 1.26 & 0.38 & 0.211 & $1.30 \mathrm{~h}$ \\
\hline IBMPG3 & 0.122 & $6.8 \%$ & 12.0 & 1.27 & 0.30 & 0.198 & 1.000 & $1.95 \mathrm{~h}$ \\
\hline IBMPG4 & 0.132 & $7.4 \%$ & 13.5 & 1.31 & 0.36 & 0.092 & 1.000 & $39.1 \mathrm{~m}$ \\
\hline IBMPG5 & 0.086 & $4.8 \%$ & 14.0 & 1.41 & 0.43 & 0.100 & 0.996 & $1.23 \mathrm{~h}$ \\
\hline IBMPG6 & 0.103 & $5.7 \%$ & 13.6 & 1.30 & 0.32 & 0.135 & 1.000 & $1.69 \mathrm{~h}$ \\
\hline IBMPGNEW1 & 0.168 & $9.4 \%$ & 19.5 & 1.51 & 0.33 & 0.029 & 1.000 & $1.84 \mathrm{~h}$ \\
\hline IBMPGNEW2 & 0.139 & $7.7 \%$ & 11.9 & 1.17 & 0.30 & 0.280 & 1.000 & $4.07 \mathrm{~m}$ \\
\hline G1 & 0.040 & $3.6 \%$ & 13.2 & 1.30 & 0.39 & 0.198 & 0.953 & $9.38 \mathrm{~m}$ \\
\hline G2 & 0.050 & $4.6 \%$ & 12.0 & 1.17 & 0.36 & 0.280 & 0.967 & $19.58 \mathrm{~m}$ \\
\hline G3 & 0.055 & $5.0 \%$ & 13.6 & 1.25 & 0.36 & 0.200 & 0.986 & $55.59 \mathrm{~m}$ \\
\hline G4 & 0.050 & $4.6 \%$ & 15.3 & 1.27 & 0.31 & 0.149 & 0.988 & $2.41 \mathrm{~h}$ \\
\hline G5 & 0.044 & $4.0 \%$ & 13.2 & 1.26 & 0.31 & 0.125 & 0.998 & \\
\hline
\end{tabular}

Table 5.1: Survival probability Estimation of power grids for user-provided values of $y$ with $95 \%$ confidence and maximum absolute error of \pm 0.05 .

is earlier. This modification makes the run-time dependent on $y$; for a given grid, the time required to estimate the survival probability using mesh model increases with increase in $y$, but it also enables us to estimate the survival probability of the grid $\forall t \leq y$.

Table 5.1 compares the estimated survival probabilities of the test grids using series model and mesh model. It can be easily observed that by taking redundancies into account, mesh model consistently predicts a far more reliable grid than the series model in every case and thus supports the claim that a lot of EM-margin is left 'on the table'. Note that the CPU time reported in the last column of table 5.1 is the time required to complete 490 trials (iterations), the average time per iteration is much less. For example, in grid G5, that has $\sim 1$ million nodes, the average time per trial is only $17.74 s$ when $y=13.2$ yrs.

A complete statistical analysis was done for the IBMPG5 grid to estimate the RVs $\mathbf{T}_{m}$ and $\mathbf{T}_{s}$. The results are as shown in figure 5.6. A total of 490 grid TTF samples were obtained for each model. Using the goodness-of-fit methods [21], it was found that the samples could be fitted well by a normal distribution (figure 5.6a), so that $\mathbf{T}_{m}$ and $\mathbf{T}_{s}$ have a normal distribution. This is further verified by the plots in figure $5.6 \mathrm{~b}, \mathrm{c}$ and $\mathrm{d}$, which show a very good agreement between the empirical PDF, Reliability and failure rate functions and the actual curves plotted from expressions (2.17), (2.20) and (2.21) with parameter values as obtained from the goodnessof-fit plot. Note that since $\mathbf{T}_{m}$ and $\mathbf{T}_{s}$ are shown to have a normal distribution, the use of (2.48) as a stopping criterion to estimate MTF is also verified. 

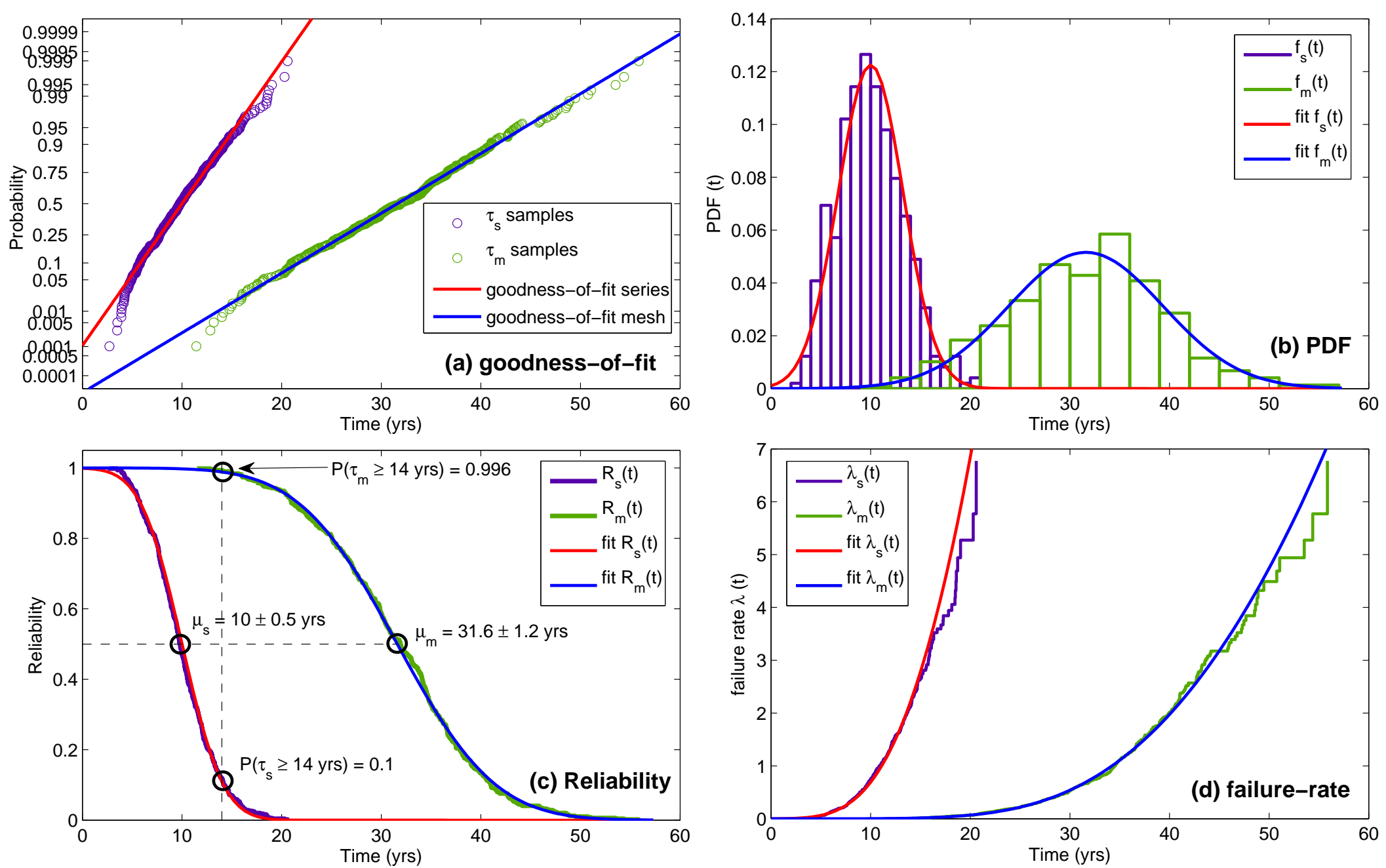

Figure 5.6: Estimated statistics of RVs $\mathbf{T}_{s}$ and $\mathbf{T}_{m}$ for IBMPG5, they are found to have a normal distribution 
Table 5.2: Speed and accuracy after using selective TTF update approach to estimate power grid MTF, $\overline{|\pi(\mathrm{R})|}$ is the average size of the set $\pi(\mathrm{R})$ (in terms of \% of interconnects) over all failures when $\delta_{v}^{\star}=10^{-3} V$ and $\epsilon^{+}$is the percentage error in MTF estimation with respect to the original approach.

\begin{tabular}{|c|c|c|c|c|c|c|c|c|c|c|c|}
\hline \multirow{3}{*}{$\begin{array}{l}\text { Grid } \\
\text { Name }\end{array}$} & \multirow{3}{*}{$\begin{array}{l}\max \left(v_{0}\right) \\
\left(\begin{array}{ll}\% & v_{d d}\end{array}\right)\end{array}$} & \multicolumn{7}{|c|}{ Mesh Model with selective updates, $\delta_{v}^{\star}=10^{-3} \mathrm{~V}$} & \multicolumn{3}{|c|}{ Comparision } \\
\hline & & \multirow{2}{*}{$\begin{array}{c}\mu_{m}^{+} \\
(\mathrm{yrs})\end{array}$} & \multirow{2}{*}{$\begin{array}{c}\# \mathrm{MC} \\
w_{m}\end{array}$} & \multirow{2}{*}{$\begin{array}{c}\text { \#Fails } \\
\overline{k_{f}}\end{array}$} & \multirow{2}{*}{$\begin{array}{c}\text { Time/Fail } \\
\overline{t_{f}}\end{array}$} & \multirow{2}{*}{$\begin{array}{c}\text { Time/MC } \\
\overline{t_{m c}}\end{array}$} & \multirow{2}{*}{$\begin{array}{c}\text { CPU-Time } \\
t_{c p u}\end{array}$} & \multirow[t]{2}{*}{$\overline{\overline{|\pi(\mathrm{R})|}}$} & \multirow[t]{2}{*}{$\varepsilon^{+}$} & \multicolumn{2}{|c|}{ Speed-up in } \\
\hline & & & & & & & & & & $\overline{t_{f}}$ & $t_{\text {cpu }}$ \\
\hline IBMPG2 & $7.0 \%$ & 34.17 & 51 & 19.92 & $0.18 \mathrm{~s}$ & $3.61 \mathrm{~s}$ & $3.07 \mathrm{~m}$ & $21.77 \%$ & $\uparrow 0.32 \%$ & $1.62 \mathrm{x}$ & $1.62 \mathrm{x}$ \\
\hline IBMPG3 & $6.8 \%$ & 39.62 & 78 & 45.26 & $2.18 \mathrm{~s}$ & $1.64 \mathrm{~m}$ & $2.14 \mathrm{~h}$ & $6.50 \%$ & $\downarrow 0.97 \%$ & $1.39 \mathrm{x}$ & $1.59 \mathrm{x}$ \\
\hline IBMPG4 & $7.4 \%$ & 35.70 & 92 & 37.86 & $2.47 \mathrm{~s}$ & $1.56 \mathrm{~m}$ & $2.38 \mathrm{~h}$ & $7.82 \%$ & $\downarrow 5.18 \%$ & $1.55 \mathrm{x}$ & $0.95 \mathrm{x}$ \\
\hline IBMPG5 & $4.8 \%$ & 31.97 & 96 & 17.26 & $0.84 \mathrm{~s}$ & $14.52 \mathrm{~s}$ & $23.23 \mathrm{~m}$ & $15.47 \%$ & $\downarrow 1.16 \%$ & $1.89 \mathrm{x}$ & $1.76 \mathrm{x}$ \\
\hline IBMPG6 & $5.7 \%$ & 43.45 & 58 & 34.33 & $1.02 \mathrm{~s}$ & $35.05 \mathrm{~s}$ & $33.88 \mathrm{~m}$ & $12.95 \%$ & $\uparrow 3.63 \%$ & $3.22 \mathrm{x}$ & $4.22 \mathrm{x}$ \\
\hline IBMPGNEW1 & $9.4 \%$ & 59.12 & 108 & 63.91 & $1.31 \mathrm{~s}$ & $1.39 \mathrm{~m}$ & $2.50 \mathrm{~h}$ & $2.82 \%$ & $\downarrow 1.41 \%$ & $2.11 \mathrm{x}$ & $1.71 \mathrm{x}$ \\
\hline IBMPGNEW2 & $7.7 \%$ & 39.50 & 73 & 42.26 & $4.02 \mathrm{~s}$ & $2.83 \mathrm{~m}$ & $3.44 \mathrm{~h}$ & $7.09 \%$ & $\downarrow 0.36 \%$ & $1.33 \mathrm{x}$ & $1.36 \mathrm{x}$ \\
\hline G1 & $3.6 \%$ & 33.88 & 51 & 24.10 & $0.08 \mathrm{~s}$ & $2.03 \mathrm{~s}$ & $1.72 \mathrm{~m}$ & $0.081 \%$ & $\uparrow 0.73 \%$ & $2.50 \mathrm{x}$ & $4.27 \mathrm{x}$ \\
\hline G2 & $4.6 \%$ & 33.75 & 93 & 34.28 & $0.31 \mathrm{~s}$ & $10.62 \mathrm{~s}$ & $16.47 \mathrm{~m}$ & $0.043 \%$ & $\uparrow 0.14 \%$ & $1.79 \mathrm{x}$ & $1.47 \mathrm{x}$ \\
\hline G3 & $5.0 \%$ & 36.56 & 69 & 45.09 & $0.43 \mathrm{~s}$ & $19.29 \mathrm{~s}$ & $22.19 \mathrm{~m}$ & $0.025 \%$ & $\downarrow 3.46 \%$ & $2.57 \mathrm{x}$ & $3.46 \mathrm{x}$ \\
\hline G4 & $4.6 \%$ & 49.34 & 68 & 92.13 & $1.12 \mathrm{~s}$ & $1.72 \mathrm{~m}$ & $1.95 \mathrm{~h}$ & $0.015 \%$ & $\downarrow 1.17 \%$ & $2.69 \mathrm{x}$ & $2.34 \mathrm{x}$ \\
\hline G5 & $4.0 \%$ & 43.98 & 55 & 122.07 & $2.90 \mathrm{~s}$ & $5.90 \mathrm{~m}$ & $5.40 \mathrm{~h}$ & $0.006 \%$ & $\uparrow 2.50 \%$ & $2.02 \mathrm{x}$ & $1.73 \mathrm{x}$ \\
\hline \multicolumn{9}{|c|}{ Average Relative error and speed-up } & $1.75 \%$ & $2.05 x$ & $2.19 \mathrm{x}$ \\
\hline
\end{tabular}


Also, since we have a large sample size, we can have a better estimate of $E\left[\mathbf{T}_{s}\right]$ and $E\left[\mathbf{T}_{m}\right]$ for IBMPG5. From the sample mean, $E\left[\mathbf{T}_{m}\right]=31.6$ yrs and $E\left[\mathbf{T}_{s}\right]=10$ yrs. Using (2.48), we can arrive at:

$$
\sqrt{w} \frac{\left|\bar{X}_{w}\right|}{s_{w}}=\frac{z_{\alpha / 2}}{\epsilon /(1+\epsilon)}
$$

The LHS of (5.4) is known, because in this case we already have the samples. Hence, we can choose a specific $\alpha$ and find the corresponding $\epsilon$. For both the series and mesh model, we choose $\alpha=0.001$ (99.9\% confidence), which gives the maximum relative error to be $5.09 \%$ and $3.78 \%$ respectively. Thus, it can be said, with $99.9 \%$ confidence, that

$$
E\left[\mathbf{T}_{s}\right]=10 \pm 0.5 \mathrm{yrs} \quad \text { and } \quad E\left[\mathbf{T}_{m}\right]=31.6 \pm 1.2 \mathrm{yrs}
$$

Interestingly, the estimated MTF for series and mesh model, as reported in Table 4.2 is 9.96 yrs and 32.34 yrs, respectively, which falls within the range of (5.5).

Table 5.2 shows the speed and accuracy of the proposed selective TTF-update approach. A user specified value for $\delta v^{\star}$ was used for all the test grids $\left(\delta_{v}^{\star}=10^{-3} V\right)$, and the other parameters are the same as given in section 4.5. The run-time and accuracy is compared with the original approach where for each interconnect failure, the TTFs of all surviving interconnects are updated (as reported in table 4.2). The percentage error is calculated as:

$$
\varepsilon^{+} \triangleq \frac{\left(\mu_{m}^{+}-\mu_{m}^{*}\right) \times 100}{\mu_{m}^{*}}
$$

The results clearly show that with selective updates, we are able to achieve more than $2 \mathrm{x}$ speedup in both $\overline{t_{f}}$ and $t_{c p u}$ with small loss in accuracy. The maximum percentage error incurred in MTF estimation is $5.18 \%$, with the average error over all grids being only $1.75 \%$, which is very less when compared to the error in secondary approach (see table 4.3). From figure 5.2, we can observe that for a given value of $\delta_{v}^{\star},|\pi(\mathrm{R})|$ will be larger for external grids as compared to internal grids, which is confirmed from the reported values of $\overline{|\pi(\mathrm{R})|}$ in Table 5.2.

For many grids, the speed-up obtained in $\overline{t_{f}}$ for selective updates approach is higher than that of the secondary approach. On first glance this looks inconsistent, the question being: how can updating only node voltage drops be slower than updating node voltage drops and statistics for selective interconnects? The answer lies in scalability of $t_{f, k, p}$, the time taken to update voltage drops and statistics when the $k^{\text {th }}$ interconnect fails in the $p^{\text {th }}$ monte-carlo iteration. From previous theory, $t_{f, k, p}$ for selective updates approach scales as:

$$
\mathcal{O}\left(t_{f, k, p}\right)=\underbrace{\mathcal{O}\left(m^{2}\right)+\mathcal{O}(m)+\mathcal{O}(m k)+\mathcal{O}\left(k^{2}\right)}_{\text {Woodbury-Banachiewicz-Schur voltage drop updates }}+\underbrace{\mathcal{O}(1)}_{\text {updating statistics }}
$$

In the case of secondary approach, the last term is absent. Eq. (5.7) answers the question: in the secondary approach, the length of failure sequences for some grids is so large that it 

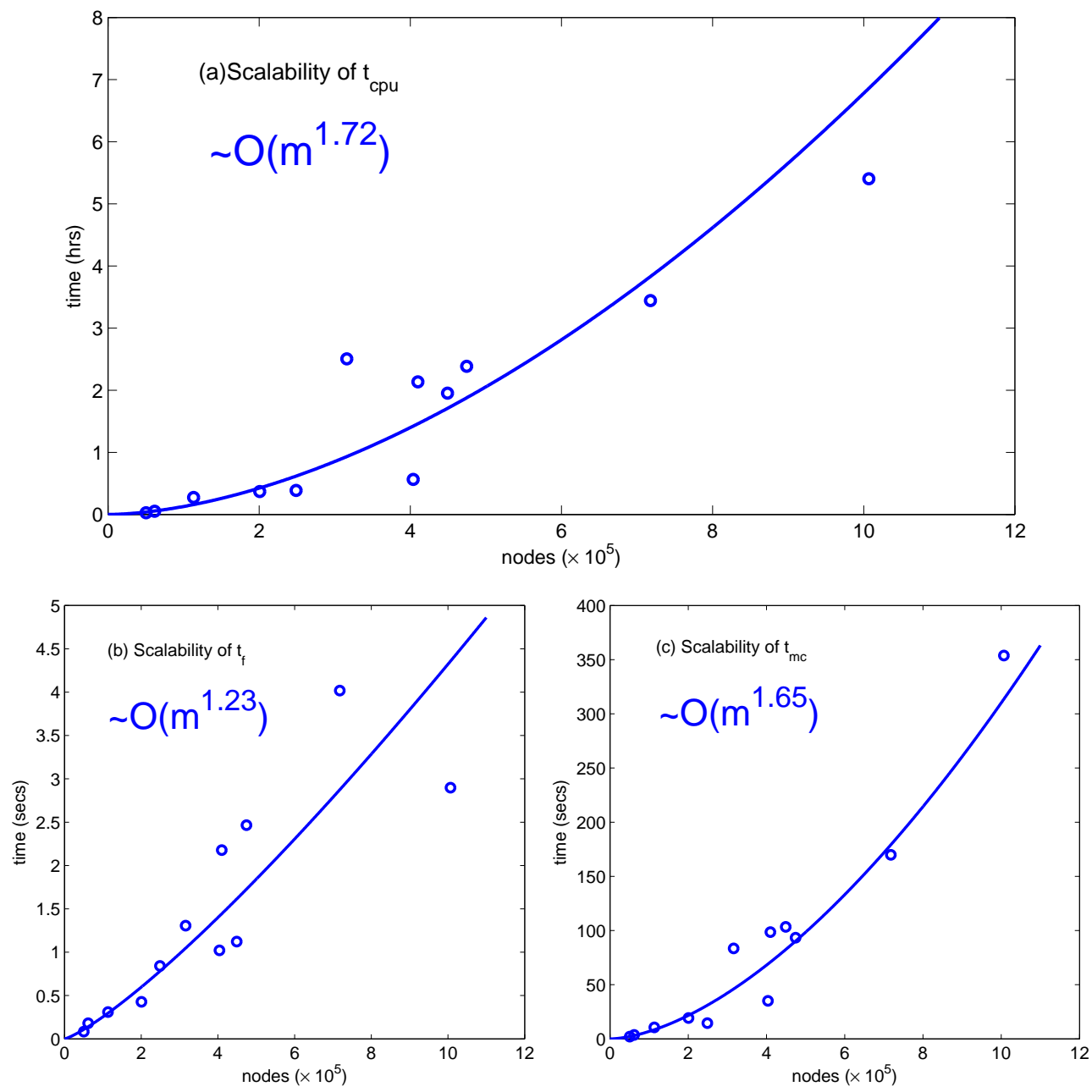

Figure 5.7: Scalability of the selective TTF update approach.

over-shadows the speed-up achieved in $\overline{t_{f}}$ by omitting the $\mathcal{O}(1)$ term. Thus, in spite of extra computation involved, the selective update approach is able to achieve greater speed-ups in $\overline{t_{f}}$. Added to that, it is able to preserve the convergence of the original approach, so that gain in $\overline{t_{f}}$ is carried over to $t_{c p u}$, resulting in overall speed-up of $2.2 \mathrm{x}$.

Although we obtain a significant speed-up in the selective updates approach, its scalability degrades when compared to the original approach. For the selective updates approach, the total time taken to estimate the MTF, $t_{c p u}$, scales as $\mathcal{O}\left(m^{1.72}\right)$, whereas in the original approach $t_{c p u}$ scales as $\mathcal{O}\left(\mathrm{m}^{1.55}\right)$. The scalability of $\overline{t_{f}}$ and $\overline{t_{m c}}$ also degrade as compared to the original approach, and are found to be $\mathcal{O}\left(m^{1.23}\right)$ and $\mathcal{O}\left(m^{1.65}\right)$, respectively, as shown in figure 5.7. The scalability deteriorates because the convergence becomes slower with increase in grid size, as evident by the increased number of Monte Carlo iterations required to estimate the MTF and longer failure sequences (for large grids) when compared to the original approach.

The constant $\delta_{v}^{\star}$ controls the speed-up vs accuracy trade-off in MTF estimation using selec- 

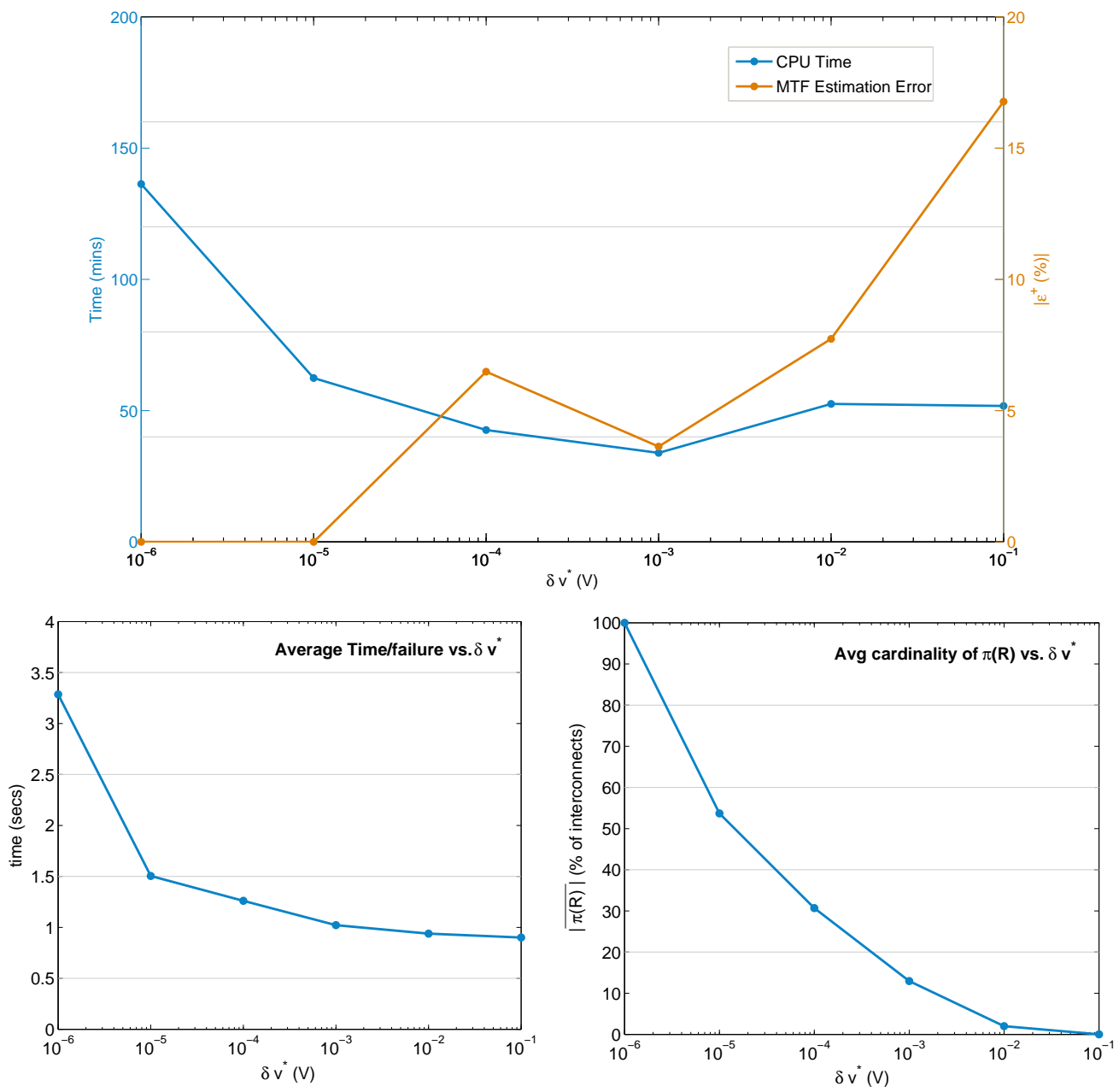

Figure 5.8: Runtime $\left(t_{\text {cpu }}\right)$ and error $\left(\left|\varepsilon^{+}\right|\right)$vs. $\delta_{v}^{\star}$; effect of varying $\delta_{v}^{\star}$ on $\overline{t_{f}}$ (average time/failure) and $\overline{|\pi(\mathrm{R})|}$ (the average cardinality of the set $\pi(\mathrm{R})$ ). All the results are for IBMPG6.

tive updates. An analysis was performed to study the extent of run-time reduction with $\delta_{v}^{\star}$ for IBMPG6. The results of the study is shown in figure 5.8. It can be seen from the figures that as $\delta_{v}^{\star}$ is increased from $10^{-6}$ to $10^{-1}, \overline{|\pi(\mathrm{R})|}$ decreases, which in turn improves $\overline{t_{f}}$ and $t_{c p u}$, at the cost of increasing the error in estimation. Even though $\overline{t_{f}}$ decreases with decreasing $\overline{|\pi(\mathrm{R})|}, t_{c p u}$ increases when $\delta v^{\star} \geq 10^{-2}$ due to decrease in convergence (as observed earlier in the secondary approach). 


\section{Chapter 6}

\section{Conclusions and Future Work}

A well-designed power grid in Integrated Circuits (ICs) not only must supply power that is free from fluctuations, but it should also guarantee that its functionality will be reliable for several years. In modern chips, the interconnect structure is arranged in several levels of metalization with thousands of inter-level connections such as via. As the modern designs become more complex and the structural dimensions of electronic interconnects become ever-smaller, the current densities continue to increase. Added to that, interconnect length has been increasing, which further decreases the reliability of interconnects. Due to these scaling trends, the designers are now faced with an ever-reducing margin between the predicted electromigration stress and the stress allowable by electromigration design rules. However, the reduction in margin for the modern designs can also be traced to the pessimism/conservatism built-in the state-of-the-art EM checking tools. A major drawback in the present EM checking tools for power grids is the series system assumption, under which a power grid is assumed to have failed if any of its interconnects fail. Clearly, the series model is highly pessimistic, considering that most modern power grids have a mesh structure.

This necessitates a EM checking tool that moves away from the overly pessimistic series model and takes into account the redundancy of the power grid while estimating its lifetime. In this work, we developed a more realistic grid EM checking and budgeting method that takes the mesh struture of the power grid. We proposed and implemented 1) the mesh model to estimate the MTF and reliability of power grid, 2) a fast and exact approach to update the voltage drops using the Woodbury formula and Banachiewicz-Schur form and 3) a framework to predict the failure statistics of a metal line as it's effective-EM current density varies over large time-scales. The mesh model accounts for the redundancies in the grid based on the new failure criteria of node voltage drop threshold, and thus obtained a more realistic estimate of grid's MTF and reliability. The results prove that the current practice of ignoring the redundancy in the grid gives a pessimistic estimate of grid TTF statistics by a factor of 3-4. We also leveraged locality to reduce the number of TTF updates to be performed after an interconnect fails. This sped- 
up our MTF estimation engine by $\sim 2 \mathrm{x}$, enabling us to estimate the MTF of a million node grid under $5.5 \mathrm{hrs}$. We also verified the stability of the reported increase in the EM margins: even when the input parameters defining the power grid configuration, property of material used etc are changed, the gain ratio never plummets down, we still get at least a $2.5 \mathrm{x}$ increase in estimated lifetime by taking the redundancies into account. However, note that since the redundancy model is applicable only to mesh structures and the signal nets hardly have such redundancy (except redundant vias), the 3-4x increase in lifetime predicted for power grids will not translate to a $3-4 \mathrm{x}$ increase in the overall chip lifetime.

There are many avenues to further extended this work. A natural extension would be to develop a budgeting framework (akin to SEB for series model) that will enable the chip level reliability to be budgeted among different class of interconnects using the mesh model. Also, since Monte Carlo iterations are highly parallelizable, one can perform these iterations using either the GPUs (CUDA/OpenCL) or cluster computing to greatly improve the run-time. Another proposed future extension could be to use a more realistic TCAD model to simulate degradation in an interconnect due to EM instead of Black's equation. It will definitely be interesting to solve the reverse problem, which can be stated as follows: given a power grid, the location of current sources and a target MTF, find the (local and global) constraints so that the power grid meets its target lifetime. 
Appendices 


\section{Appendix A}

\section{The Banachiewicz-schur form}

Let $M \in \mathbb{R}^{k \times k}$ be $2 \times 2$ block matrix:

$$
M=\left[\begin{array}{ll}
A & b \\
c^{T} & d
\end{array}\right]
$$

where $A \in \mathbb{R}^{(k-1) \times(k-1)}, b \in \mathbb{R}^{k-1}, c \in \mathbb{R}^{k-1}$, and $d$ is a scalar. The schur-complement of $A$ in $M$ is given by [56]:

$$
s=d-c^{T} A^{-1} b
$$

If both $M$ and $A$ in (A.1) are non-singular, then $s$ is non-singular too, i.e. $s \neq 0$. This allows writing $M$ as:

$$
M=\left[\begin{array}{cc}
I_{k-1} & 0 \\
c^{T} A^{-1} & I_{1}
\end{array}\right]\left[\begin{array}{cc}
A & 0 \\
0 & s
\end{array}\right]\left[\begin{array}{cc}
I_{k-1} & A^{-1} b \\
0 & I_{1}
\end{array}\right]
$$

where $I_{\lambda}$ is the identity matrix of order $\lambda$. The inverse of $M$ can now be written as [56]:

$$
M^{-1}=\left[\begin{array}{cc}
I_{k-1} & -A^{-1} b \\
0 & I_{1}
\end{array}\right]\left[\begin{array}{cc}
A^{-1} & 0 \\
0 & 1 / s
\end{array}\right]\left[\begin{array}{cc}
I_{k-1} & 0 \\
-c^{T} A^{-1} & I_{1}
\end{array}\right]
$$

which can be re-written as:

$$
M^{-1}=\left[\begin{array}{cc}
A^{-1}+\frac{A^{-1} b c^{T} A^{-1}}{s} & -\frac{A^{-1} b^{T}}{s} \\
-\frac{c^{T} A^{-1}}{s} & \frac{1}{s}
\end{array}\right]
$$

Equation (A.5) is known as the Banachiewicz-schur form. It expresses $M^{-1}$ in terms of $A^{-1}$. 


\section{Bibliography}

[1] J. Warnock, "Circuit design challenges at the 14nm technology node," in ACM/IEEE 48th Design Automation Conference (DAC-2011), San Diego, CA, June 5-9 2011, pp. 464-467.

[2] B. Geden, "Understand and avoid electromigration (EM) and IR-drop in custom IP blocks," Synopsys, White Paper, November 2011.

[3] D. F. Frost and K. F. Poole, "A method for predicting VLSI-device reliability using series models for failure mechanisms," IEEE Transactions on Reliability, vol. R-36, no. 2, pp. $234-242$, june 1987.

[4] J. Kitchin, "Statistical electromigration budgeting for reliable design and verification in a 300-mhz microprocessor," in VLSI Circuits, 1995. Digest of Technical Papers., 1995 Symposium on, jun 1995, pp. $115-116$.

[5] J. R. Black, "Electromigration- a brief survey and some recent results," IEEE Transactions on Electronic devices, vol. 16, no. 4, pp. 338-347, 1969.

[6] R. Kirchheim and U. Kaeber, "Atomistic and computer modeling of metallization failure of integrated circuits by electromigration," Journal of Applied Physics, vol. 70, no. 1, pp. 172-181, 1991. [Online]. Available: http://link.aip.org/link/?JAP/70/172/1

[7] J. Lloyd, "Electromigration failure," Journal of Applied Physics, vol. 69, no. 11, pp. 7601$7604,1991$.

[8] M. A. Korhonen, P. Borgesen, D. D. Brown, and C.-Y. Li, "Microstructure based statistical model of electromigration damage in confined line metallizations in the presence 
of thermally induced stresses," Journal of Applied Physics, vol. 74, no. 8, pp. 4995-5004, 1993. [Online]. Available: http://link.aip.org/link/?JAP/74/4995/1

[9] M. Shatzkes and J. R. Lloyd, "A model for conductor failure considering diffusion concurrently with electromigration resulting in a current exponent of 2," J. Appl. Phys., vol. 59, feb 1986.

[10] J. Clement and C. Thompson, "Modeling electromigration-induced stress evolution in confined metal lines," Journal of Applied Physics, vol. 78, no. 2, pp. 900-904, 1995.

[11] "Black's law revisited-nucleation and growth in electromigration failure," Microelectronics Reliability, vol. 47, no. 9-11, pp. 1468 -1472, 2007.

[12] J. Lloyd, "Electromigration in integrated circuit conductors," J. Phys. D: Appl. Phys., vol. 32, no. 17, pp. R109 - R118, 1999.

[13] A. Puttlitz, J. Ryan, and T. Sullivan, "Semiconductor interlevel shorts caused by hillock formation in al-cu metallization," Components, Hybrids, and Manufacturing Technology, IEEE Transactions on, vol. 12, no. 4, pp. 619-626, 1989.

[14] M. Bazu and T. Bajenescu, Failure Analysis: A Practical Guide for Manufacturers of Electronic Components and Systems. John Wiley and Sons.

[15] I. A. Blech, "Electromigration in thin aluminium on titanium nitride," Journal of Applied Physics, vol. 47, no. 4, pp. 1203-1208, 1976, doi: 10.1063/1.322842.

[16] I. A. Blech and C. Herring, "Stress generation by electromigration," Applied Physics Letters, vol. 29, no. 3, pp. 131-133, 1976. [Online]. Available: http://link.aip.org/link/?APL/29/131/1

[17] I. A. Blech and K. L. Tai, "Measurement of stress gradients generated by electromigration," Applied Physics Letters, vol. 30, no. 8, pp. 387-389, 1977. [Online]. Available: http://link.aip.org/link/?APL/30/387/1 
[18] C. Herring, "Diffusional viscosity of a polycrystalline solid," Journal of Applied Physics, vol. 21, no. 5, pp. 437-445, 1950. [Online]. Available: http://link.aip.org/link/?JAP/21/437/1

[19] J. M. Towner and E. P. Van de Ven, "Aluminum electromigration under pulsed d.c. conditions," in Reliability Physics Symposium, 1983. 21st Annual, 1983, pp. 36-39.

[20] K. Lee, "Electromigration recovery and short lead effect under bipolar- and unipolar-pulse current," in IEEE International Reliability Physics Symposium (IRPS), april 2012, pp. 6B.3.1 -6B.3.4.

[21] E. A. Amerasekera and F. N. Najm, Failure Mechanisms in Semiconductor Devices, 2nd ed. John Wiley and Sons, 1998.

[22] NIST/SEMATECH, "e-Handbook of Statistical Methods," May 2004. [Online]. Available: http://www.itl.nist.gov/div898/handbook/index.htm

[23] J. D. Venables and R. G. Lye, "A statistical model for electromigration induced failure in thin film conductors," in Reliability Physics Symposium, 1972. 10th Annual, 1972, pp. $159-164$.

[24] M. Gall, P. Ho, C. Capasso, D. Jawarani, R. Hernandez, and H. Kawasaki, "Electromigration early failure distribution in submicron interconnects," in Interconnect Technology, 1999. IEEE International Conference, 1999, pp. 270-272.

[25] J. Lloyd and J. Kitchin, "The electromigration failure distribution: The fineline case," Journal of Applied Physics, vol. 69, no. 4, pp. 2117-2127, 1991.

[26] G. Yoh and F. Najm, "A statistical model for electromigration failures," in Quality Electronic Design, 2000. ISQED 2000. Proceedings. IEEE 2000 First International Symposium on, 2000, pp. 45-50.

[27] J. E. F. I. R. Miller and R. Johnson., Probability and Statistics for Engineers, 6th ed. Prentice-Hall, Inc., 2010. 
[28] J. Cho and C. V. Thompson, "Grain size dependence of electromigration induced failures in narrow interconnects," Journal of Applied Physics, no. 54, pp. 2577-2579, 1989.

[29] M. Lin, M. Lin, and T. Wang, "Effects of length scaling on electromigration in dual-damascene copper interconnects," Microelectronics Reliability, vol. 48, no. 4, pp. 569 - 577, 2008. [Online]. Available: http://www.sciencedirect.com/science/article/pii/S0026271407004568

[30] S. M. Alam, C. L. Gan, C. V. Thompson, and D. E. Troxel, "Reliability computer-aided design tool for full-chip electromigration analysis and comparison with different interconnect metallizations," Microelectronics Journal, vol. 38, no. 4-5, pp. 463-473, 2007. [Online]. Available: http://www.sciencedirect.com/science/article/pii/S0026269206002849

[31] S. Alam, F. L. Wei, C. L. Gan, C. V. Thompson, and D. Troxel, "Electromigration reliability comparison of cu and al interconnects," in Quality of Electronic Design, 2005. ISQED 2005. Sixth International Symposium on, 2005, pp. 303-308.

[32] G. Marsaglia and W. W. Tsang, "The ziggurat method for generating random variables," Journal of Statistical Software, vol. 5, no. 8, pp. 1-7, 10 2000. [Online]. Available: http://www.jstatsoft.org/v05/i08

[33] R. A. R. L. T. Pillage and C. Visweswariah, Electronic Circuit and System Simulation methods. New York, NY: McGraw Hill, 1995.

[34] Y. Saad, Iterative Methods for Sparse Linear Systems. Philadelphia, PA: SIAM, 2003.

[35] D. Kouroussis and F. N. Najm, "A static pattern-independent technique for power grid voltage integrity verification," in ACM/IEEE 40th Design Automation Conference, Anaheim, CA, Jun 2003, pp. 99-104.

[36] F. N. Najm, "Statistical estimation of the signal probability in VLSI circuits," University of Illinois at Urbana-Champaign, Coordinated Science Laboratory, Tech. Rep. UILU-ENG93-2211, April 1993. 
[37] M. A. Korhonen, P. Borgesen, K. N. Tu, and C.-Y. Li, "Stress evolution due to electromigration in confined metal lines," J. Appl. Phys., vol. 73, no. 8, pp. $3790-3799$, apr 1993.

[38] S. Rochel, G. Steele, J. Lloyd, S. Hussain, and D. Overhauser, "Full-chip reliability analysis," in Reliability Physics Symposium Proceedings, 1998. 36th Annual. 1998 IEEE International, 1998, pp. 356-362.

[39] B.-K. Liew, P. Fang, N. W. Cheung, and C. Hu, "Circuit reliability simulator for interconnect, via, and contact electromigration," Electron Devices, IEEE Transactions on, vol. 39, no. 11, pp. 2472-2479, 1992.

[40] J. Tao, N. W. Cheung, and C. Hu, "Metal electromigration damage healing under bidirectional current stress," Electron Device Letters, IEEE, vol. 14, no. 12, pp. 554-556, 1993.

[41] — - "An electromigration failure model for interconnects under pulsed and bidirectional current stressing," Electron Devices, IEEE Transactions on, vol. 41, no. 4, pp. 539-545, 1994.

[42] L. Doyen, E. Petitprez, P. Waltz, X. Federspiel, L. Arnaud, and Y. Wouters, "Extensive analysis of resistance evolution due to electromigration induced degradation," Journal of Applied Physics, vol. 104, no. 12, p. 123521, 2008. [Online]. Available: http://link.aip.org/link/?JAP/104/123521/1

[43] R. Ahmadi and F. N. Najm, "Timing analysis in presence of power supply and ground voltage variations," in IEEE/ACM International Conference on Computer-Aided Design, San Jose, CA, Nov 2003, pp. 176-183.

[44] D. Blaauw, S. Pant, R. Chaudhry, and R. Panda, Electronic Design Automation for Integrated Circuits Handbook. CRC Press, ch. 24.

[45] F. N. Najm, Circuit Simulation, 1st ed. John Wiley and Sons, 2010, ch. 2.

[46] S. Osnaga, "On rank one matrices and invariant subspaces," Balkan Journal of Geometry and Its Applications, vol. 10, no. 1, pp. 145-148, 2005. 
[47] N. J. Higham, Functions of Matrices: theory and computation, 1st ed. SIAM, 2008.

[48] T. A. Davis, "A column pre-ordering strategy for the unsymmetric-pattern multifrontal method," ACM Trans. Math. Softw., vol. 30, no. 2, pp. 165-195, Jun. 2004. [Online]. Available: http://doi.acm.org/10.1145/992200.992205

[49] ——, "Algorithm 832: Umfpack v4.3-an unsymmetric-pattern multifrontal method," ACM Trans. Math. Softw., vol. 30, no. 2, pp. 196-199, Jun. 2004. [Online]. Available: http://doi.acm.org/10.1145/992200.992206

[50] T. A. Davis and I. S. Duff, "A combined unifrontal/multifrontal method for unsymmetric sparse matrices," ACM Trans. Math. Softw., vol. 25, no. 1, pp. 1-20, Mar. 1999. [Online]. Available: http://doi.acm.org/10.1145/305658.287640

[51] T. Davis and I. Duff, "An unsymmetric-pattern multifrontal method for sparse lu factorization," SIAM Journal on Matrix Analysis and Applications, vol. 18, no. 1, pp. 140-158, 1997. [Online]. Available: http://epubs.siam.org/doi/abs/10.1137/S0895479894246905

[52] S. R. Nassif, "Power grid analysis benchmarks," in ASP-DAC, 2008, pp. 376-381.

[53] "Failure mechanisms and models for semiconductor devices," Joint Electron Devices Engineering Council (JEDEC), Tech. Rep. JEP122F, 2010.

[54] S. M. Alam, "Design tool and methodologies for interconnect reliability analysis in integrated circuits," Ph.D. dissertation, MASSACHUSETTS INSTITUTE OF TECHNOLOGY, 2004.

[55] J. Jaffari and M. Anis, "Statistical thermal profile considering process variations: Analysis and applications," Computer-Aided Design of Integrated Circuits and Systems, IEEE Transactions on, vol. 27, no. 6, pp. 1027-1040, 2008.

[56] Y. Tian and Y. Takane, "Schur complements and banachiewicz-schur forms," Electronic Journal of Linear Algebra, vol. 13, pp. 405-418, Dec 2005. 